\title{
The Food Additive Xanthan Gum Drives Adaptation of the Human Gut Microbiota
}

2

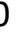

*Matthew P. Ostrowski ${ }^{1}$, *Sabina Leanti La Rosa ${ }^{2,3}$, Benoit J. Kunath ${ }^{2}$, Andrew Robertson ${ }^{4}$, Gabriel Pereira $^{1}$, Live H. Hagen ${ }^{2}$, Neha J. Varghese ${ }^{5}$, Ling Qiu ${ }^{1}$, Tianming Yao ${ }^{6}$, Gabrielle Flint $^{1}$, James Li ${ }^{7}$, Sean McDonald ${ }^{7}$, Duna Buttner ${ }^{1}$, Nicholas A. Pudlo ${ }^{1}$, Matthew K. Schnizlein ${ }^{1}$, Vincent B. Young ${ }^{1}$, Harry Brumer ${ }^{7}$, Thomas Schmidt ${ }^{1}$, Nicolas Terrapon ${ }^{8,9}$, Vincent Lombard ${ }^{8,9}$, Bernard Henrissat ${ }^{8,9,10}$, Bruce Hamaker ${ }^{6}$, Emiley A Eloe-Fadrosh ${ }^{5}$, Ashootosh Tripathi ${ }^{4}$, ^Phillip B. Pope ${ }^{2,3}, \wedge$ Eric Martens ${ }^{1}$

${ }^{1}$ Department of Microbiology and Immunology, University of Michigan, Ann Arbor, MI 48109, USA

${ }^{2}$ Faculty of Chemistry, Biotechnology and Food Science, Norwegian University of Life Sciences, Aas N-1433 Norge, Norway.

${ }^{3}$ Faculty of Biosciences, Norwegian University of Life Sciences, Aas N-1433 Norge, Norway. ${ }^{4}$ Life Sciences Institute: Natural Products Discovery Core, University of Michigan, Ann Arbor, MI 48109, USA

${ }^{5}$ DOE Joint Genome Institute, Berkeley, CA, USA

${ }^{6}$ Department of Food Science and Whistler Center for Carbohydrate Research, Purdue University, West Lafayette, IN 47907, USA

${ }^{7}$ Michael Smith Laboratories, University of British Columbia, 2185 East Mall, Vancouver, BC, V6T $1 Z 4$

${ }^{8}$ Centre National de la Recherche Scientifique, Aix-Marseille Univ., UMR7257 AFMB, Marseille, France

${ }^{9}$ Institut National de Recherche pour l'Agriculture, l'Alimentation et l'Environnement, USC1408 AFMB, Marseille, France

${ }^{10}$ Department of Biological Sciences, King Abdulaziz University, Jeddah, Saudi Arabia

* These authors contributed equally to this work

^ Correspondence to: phil.pope@nmbu.no, emartens@umich.edu 


\section{Summary}

33 The diets of industrialized countries reflect the increasing use of processed foods, often with the introduction of novel food additives. Xanthan gum is a complex polysaccharide with unique rheological properties that have established its use as a widespread stabilizer and thickening agent $^{1}$. However, little is known about its direct interaction with the gut microbiota, which plays a central role in digestion of other, chemically-distinct dietary fiber polysaccharides. Here, we show that the ability to digest xanthan gum is surprisingly common in industrialized human gut microbiomes and appears to be contingent on the activity of a single bacterium that is a member of an uncultured bacterial genus in the family Ruminococcaceae. We used a combination of enrichment culture, multi-omics, and recombinant enzyme studies to identify and characterize a complete pathway in this uncultured bacterium for the degradation of xanthan gum. Our data reveal that this keystone degrader cleaves the xanthan gum backbone with a novel glycoside hydrolase family 5 (GH5) enzyme before processing the released oligosaccharides using additional enzymes. Surprisingly, some individuals harbor a Bacteroides species that is capable of consuming oligosaccharide products generated by the keystone Ruminococcaceae or a purified form of the GH5 enzyme. This Bacteroides symbiont is equipped with its own distinct enzymatic pathway to cross-feed on xanthan gum breakdown products, which still harbor the native linkage complexity in xanthan gum, but it cannot directly degrade the high molecular weight polymer. Thus, the introduction of a common food additive into the human diet in the past 50 years has promoted the establishment of a food chain involving at least two members of different phyla of gut bacteria.

\section{Introduction}

Evidence is accumulating that food additives impact the symbiosis between humans and their associated gut microbiomes, in some cases promoting intestinal inflammation and metabolic syndrome ${ }^{2}$ or promoting certain pathogens ${ }^{3}$. Often used as thickeners and emulsifiers, polysaccharides are a prominent subset of these food additives. Since dietary polysaccharides other than starch typically transit the upper intestinal tract undigested, polysaccharide-based additives can potentially exert their influence by altering the composition and function of the microbiome, which can in turn impact host health ${ }^{4,5}$. Given generally regarded as safe (GRAS) approval by the United States Food and Drug Administration in 1968, xanthan gum (XG) is an 
exopolysaccharide produced by the bacterium Xanthamonas campestris that has been increasingly used in the food supply for the last 50 years. This polymer has the same $\beta-1,4-$ linked backbone as cellulose, but contains trisaccharide branches on alternating glucose residues consisting of $\alpha$-1,3-mannose, $\beta$-1,2-glucuronic acid, and terminal $\beta$-1,4-mannose (Figure 1a). The terminal $\beta$-D-mannose and the inner $\alpha$-D-mannose are variably pyruvylated at the 4,6position or acetylated at the 6-position, respectively, with amounts determined by specific $X$. campestris strains and culture conditions ${ }^{6}$. XG is typically added at concentrations of $0.05-0.5 \%$ to foods including bakery products, condiments, and ice cream ${ }^{7}$. XG is also used as a replacement for gluten in a gluten-free diet, which is a vital component for limiting intestinal inflammation in patients with celiac disease, a lifelong condition estimated to affect $0.7-1.4 \%$ of the population and increasing in prevalence ${ }^{8}$. In gluten-free baked goods, XG can be consumed in up to gram quantities per serving. Although small doses of $\mathrm{XG}$ have not been connected to immediate health impacts, its fate in the digestive tract is unknown ${ }^{9}$. The low-level, but constant consumption of XG by a large portion of the population in the industrialized world and its higher intake by specific subpopulations highlight the need to understand the effects of this polysaccharide food additive on the ecology of the human gut microbiota.

\section{A member of an uncultured bacterial genus is a keystone XG degrader in the human gut microbiome}

To identify potential XG degrading bacteria in the human gut microbiome, we surveyed a group of healthy 18-20 year-old adults using a bacterial enrichment culture strategy in which a partially defined minimal medium was combined with XG as the main carbon source ${ }^{10}$. This medium was inoculated directly with feces that had been collected in anaerobic preservation buffer within $24 \mathrm{~h}$ prior to testing and cultures containing individual samples were passaged 3 times with 1-2 days of growth in between. Growth of the final passage was monitored quantitatively for bacterial growth or for loss of the gel-like viscosity that is characteristic of XG in solution. We originally found one XG-degrading culture (see Materials and Methods) and in an expanded experiment in which 60 individuals were sampled we identified 30 positive cultures, indicating that the ability of intestinal bacteria to degrade $\mathrm{XG}$ is unexpectedly common among the population surveyed. 
Experiments with a culture derived from one positive subject revealed that bacterial growth depended on the amount of XG provided in the medium, demonstrating specificity for this nutrient (Figure 1b). Attempts to enrich the causal XG-consuming organism(s) with additional passaging (total of 10-20 times) consistently yielded stable mixed microbial cultures that contained multiple operational taxonomic units (OTUs; between 12-22 OTUs per culture with relative abundance $\geq 0.5 \%$ ) (Figure 1c, 1 d). While these cultures had commonalities at the genus level, there was surprisingly only one OTU that was $\geq 0.5 \%$ and common across all 21 enrichment cultures examined. This common OTU was identified as a member of Ruminococcaceae uncultured genus 13 (R. UCG13) in the Silva database ${ }^{11}$ (Figure 1d, commonly used anaerobic solid medium (brain-heart infusion with $10 \%$ horse blood) resulted in loss of two previously abundant Gram-positive OTUs (loss defined as $<0.01 \%$ relative abundance), which included the R. UCG13 OTU and corresponded with loss of the XGdegrading phenotype when plate-passaged bacteria were re-inoculated into medium with XG (Figure 1c).

Despite R. UCG13 and a Bacteroides OTU being present at $>20 \%$ relative abundance in the original 12 OTU community, we repeatedly failed to isolate pure cultures that could degrade XG using different solid media that are effective for Gram-positive and -negative bacteria (the abundant Bacteroides OTU was captured multiple times, while R. UCG13 was never isolated). Dilution of the active 12-OTU community to extinction in medium supplemented with either XG or an equal amount of its component monosaccharides, resulted in loss of growth on XG at higher dilutions than simple sugars (Extended Data 2). This observation suggests that the ability to degrade XG in the medium conditions we employed requires multiple OTUs to be present (i.e., diluted into the same well together), which could be explained by either multiple species being directly involved or the necessity of other species to promote sufficient growth conditions for the XG degrader ${ }^{12}$. Collectively, these results suggest that a member of an uncultured Ruminococcaceae genus is necessary for XG degradation but may be unable to grow in isolation 
To identify XG-degrading genes within our bacterial consortium, we performed combined metagenomics and metatranscriptomics analysis on the original XG-degrading culture, using samples harvested throughout growth in liquid medium with XG (Extended Data 3). From these samples, we reconstructed 18 metagenome assembled genomes (MAGs), 7 that were high quality (completion $>90 \%$ and contamination $<5 \%$ ) (Supplemental Table 2). To connect $16 \mathrm{~S}$ rRNA genes to MAGs, we performed additional long-read sequencing that yielded 2 MAGs that were complete circular chromosomes and one of these was identified as R. UCG13 (with four complete 16S rRNA operons, three of which were identical to the R. UCG13 OTU). This circular R. UCG13 genome was distantly related (47.26\% Average Amino Acid Identity, AAI) to the recently cultured bacterium Monoglobus pectinolyticus ${ }^{13}$. Annotation of carbohydrate-active enzymes (CAZymes) in the R. UCG13 MAG revealed a single locus encoding several highly expressed enzymes that are candidates for XG degradation (Figure 2, Extended Data 3). These included a polysaccharide lyase family 8 (PL8) with distant homology to known xanthan lyases from soil bacteria Paenibacillus alginolyticus XL-1 ${ }^{14}$ (36\% identity/73\% coverage) and Bacillus sp. $\mathrm{GL}^{15}$ (32\% identity/81\% coverage; Figure 2). Xanthan lyases typically remove the terminal pyruvylated mannose prior to depolymerization of the $\beta$-1,4-glucose backbone, leaving a 4,5 unsaturated residue at the glucuronic acid position, although some tolerate non-pyruvylated mannose ${ }^{16,17}$. This same locus also contained two GH5 enzymes, a family that includes endoglucanases and xyloglucanases, with the potential to cleave the xanthan gum backbone ${ }^{18}$, a GH88 to remove the unsaturated glucuronic acid residue produced by the PL $8^{19}$, and two GH38s which could potentially cleave the $\alpha$-D-mannose ${ }^{20}$. Two carbohydrate esterases (CEs) could potentially remove the acetylation from the mannose ${ }^{21}$. Secretion signal prediction detected possible signal peptidase I (SPI) motifs for the two GH5s and one of the CEs (CE-A), while the other enzymes lacked detectable membrane localization and secretion signals ${ }^{22}$. In addition to putative enzymes to cleave the glycosidic bonds contained within xanthan gum, this locus also contained proteins predicted to be involved in sensing, binding, and transporting sugars and oligosaccharides.

Co-localization and expression of genes that saccharify a common polysaccharide into discrete polysaccharide utilization loci (PULs) is common in the Gram-negative Bacteroidetes ${ }^{23}$. Although not present in most XG-degrading cultures, we obtained a second circular MAG affiliated to $B$. intestinalis, which was conspicuously the most abundant OTU (up to $\sim 50 \%$ ) in 
the mixed species culture that it was derived from (Figure 1). This MAG contained a putative PUL that was highly expressed during growth on XG (Figure 2, Extended Data 3) and encodes hallmark SusC-/SusD-like proteins, a sensor/regulator, and predicted GH88, GH92 and GH3 enzymes, which could potentially cleave the unsaturated $\beta$-glucuronyl, $\alpha$-mannosyl, and $\beta$ glucosyl linkages in XG, respectively. Like the candidate gene cluster in R. UCG13, this PUL also contained a GH5 enzyme, which was assigned to subfamily GH5_5. Finally, a putative polysaccharide lyase (PL) was predicted, remotely related to alginate lyases ${ }^{24,25}$, as a candidate for removing the terminal mannose. In addition to the lyase domain, this multi-modular protein contains a carbohydrate esterase domain (CE) that could remove the acetyl groups positioned on the mannose. Extensive work has been conducted to characterize the substrate-specificity of PULs, which is demonstrated by hundreds of genomes with characterized and predicted PULs in the PUL database (PUL-DB) ${ }^{26}$. However, this database only harbored a single genome with a partially related homolog of the B. intestinalis PUL (B. salyersiae WAL 10018 PUL genes HMPREF1532_01924-HMPREF1532_01938), highlighting the diversity of polysaccharide utilization machinery that remains for discovery and characterization.

Interestingly, neutral monosaccharide analysis from our XG-degrading culture showed a relatively stable 1:1 ratio of glucose:mannose in the residual polysaccharide in the culture over time, implying that lyase-digested xanthan gum was not accumulating as growth progressed (Extended Data 3). This could be due to fast depolymerization and cellular importation of the $\mathrm{XG}$ polymer following lyase removal of the terminal mannose. Alternatively, the data are also consistent with a degradation model in which the XG backbone is cleaved prior to subsequent hydrolysis of the repeating pentasaccharide, a pathway that has not been characterized in XGdegrading bacteria and might result in pentasaccharides with full linkage complexity being transported into the bacterial cell before depolymerization ${ }^{27,28}$.

\section{R. UCG13 produces a unique endo-acting xanthanase activity}

To investigate the cellular location of the enzymes responsible for xanthan degradation, the original 12-OTU culture was grown in XG medium and separated into filtered cell-free supernatant, cells that were washed to remove supernatant and resuspended or lysed, or lysed cells with supernatant. Incubation of these fractions with $\mathrm{XG}$ and subsequent analysis by thin layer chromatography (TLC) revealed that the cell-free supernatant was capable of 
depolymerizing XG into large oligosaccharides, while the intracellular fraction was required to further saccharify these products into smaller components (Extended Data 4). Liquid chromatography-mass spectrometry (LC-MS) analysis of cell-free supernatant incubated with $\mathrm{XG}$ revealed the presence of pentameric and decameric oligosaccharides matching the structure of xanthan gum (Figure 3a), supporting the model described above in which secreted endocleaving enzymes first hydrolyze native XG before xanthan lyase and other enzymes cleave the attached sidechains.

To identify enzymes responsible for XG hydrolysis and determine their cellular location, we grew three independent cultures in liquid medium containing XG and subjected cell-free supernatants to ammonium sulfate precipitation. Each of the resuspended protein preparations was able to hydrolyze XG as demonstrated by a complete loss of viscosity after overnight incubation. We proceeded to fractionate each sample with various purification methods (defined in methods), collecting and pooling xanthan-degrading fractions for subsequent purification steps and taking three different purification paths (Extended Data 5). Interestingly, the most pure sample obtained ran primarily as a large smear when loaded onto an SDS-PAGE gel, but separated into distinct bands after boiling, suggesting the formation of a multimeric protein complex, which is reminiscent of cellulosomes or other complexes ${ }^{29}$ (Extended Data 5). Proteomic analysis of the samples from the three different activity-guided fractionation experiments yielded 33 proteins present across all three experiments, including 22 from R. UCG13, 11 of which were annotated as CAZymes (Extended Data 5, Supplemental Table 3). While most of the proteins were either detected in low amounts or lacked functional predictions consistent with polysaccharide degradation, one of the most abundant proteins across all three samples was one of two GH5 enzymes $(R u G H 5 a)$ encoded in the previously identified R. UCG13 candidate xanthan locus.

$R u \mathrm{GH} 5 \mathrm{a}$ consists of an $\mathrm{N}$-terminal signal peptide sequence, its main catalytic domain (which does not classify into any of the GH5 subfamilies), and 3 tandem carbohydrate-binding modules (CBMs), which are often associated with CAZymes and can facilitate polysaccharide degradation (Figure 3b) ( $^{30}$. The protein also contains a significant portion of undefined sequence and Listeria-Bacteroides repeat domains ( $\left.\mathrm{pfam}^{31} \mathrm{PF} 09479\right)$, a $\beta$-grasp domain originally characterized from the invasion protein InlB used by Listeria monocytogenes for host cell entry $^{32,33}$. These small repeat domains are thought to be involved in protein-protein interactions 
217 and are almost exclusively found in extracellular bacterial multidomain proteins. To test the

218 activity of $R u \mathrm{GH} 5 \mathrm{a}$ on $\mathrm{XG}$ we expressed recombinant forms of the entire protein (RuGH5a full),

219 the GH5 domain only (RuGH5a GH5-only), and the GH5 domain with either one (RuGH5a

220 GH5+CBM-A), two $(R u$ GH5a GH5+CBM-A/B), or all three of the CBMs $(R u$ GH5a

$221 \mathrm{GH} 5+\mathrm{CBMs}$, hereafter referred to as $R u \mathrm{GH} 5$ a for simplicity). All but the full-length construct

222 yielded reasonably pure proteins, but only constructs with the GH5 and all three CBMs showed

223 activity on xanthan gum, suggesting a critical role in catalysis for these CBMs. (Extended Data

224 6). The alternate $\mathrm{GH} 5(\mathrm{RuGH} 5 \mathrm{~b})$ was also expressed in a variety of forms but did not display any

225

226

227

228

229

230

231

232

233

234

235

236

237

238

239

240

241

242

243

244

245

246

247 activity on XG (Extended Data 6).

Analysis of the reaction products showed that $R u$ GH5a releases pentasaccharide repeating units of $\mathrm{XG}$, with various acetylation and pyruvylation (including di-acetylation as previously described ${ }^{34}$ ), and larger decasaccharide structures (Figure 3a). While isolation of homogenous pentameric oligosaccharides proved difficult, coincubation of XG with $R u \mathrm{GH} 5 \mathrm{a}$ and a Bacillus sp. PL8 facilitated the isolation of pure tetrasaccharide followed by in-depth 1D and 2D NMR structural characterization (Extended Data 7), which was useful in determining $R u \mathrm{GH} 5$ a regiospecificity in the $\mathrm{XG}$ backbone. Surprisingly, the NMR analysis suggested that $R u \mathrm{GH} 5 \mathrm{a}$ cleaves $\mathrm{XG}$ at the reducing end of the non-branching backbone glucosyl residue (Figure 3c, Extended Data 7). This contrasts with the product of other known xanthanases (such as the GH9 from Paenibacillus nanensis ${ }^{35}$ or the $\beta$-D-glucanase in Bacillus sp. strain GL $1^{15}$ ), which hydrolyze xanthan at the reducing end of the branching glucose, demonstrating a hitherto unknown enzymatic mechanism for the degradation of XG. While RuGH5a displayed little activity on other polysaccharides (Extended Data 6), it was able to hydrolyze both native and lyase-treated XG with comparable specificity, once more in contrast to most previously known xanthanases, which show $\geq 600$-fold preference for the lyase-treated substrate ${ }^{35}$ (Figure 3d). One exception is the xanthanase from Microbacterium sp. XT11, which also cleaves native and lyase-treated xanthan gum with similar kinetic specificity ${ }^{28}$; however, this enzyme only produces intermediate $\mathrm{XG}$ fragments from the complete polysaccharide, whereas $R u \mathrm{GH} 5 \mathrm{a}$ can cleave XG down to its repeating pentasaccharide unit. Together these data highlight the novelty of $R u \mathrm{GH} 5 \mathrm{a}$, which may be part of a multimeric protein complex in vivo and possesses a unique enzymatic mechanism and specificity. 


\section{R. UCG13 encodes all the enzymes required for XG saccharification}

In contrast to characterized PL8 ${ }^{17,36,14}$ xanthan lyases, the R. UCG13 PL8 showed no activity on the complete $\mathrm{XG}$ polymer but removed the terminal mannose from xanthan pentasaccharides produced by $R u \mathrm{GH} 5$ a (Extended Data 8). This further supports the model in which the GH5 first depolymerizes XG, followed by further saccharification of the XG repeating unit, likely inside the cell. Both R. UCG13 carbohydrate esterases were able to remove acetyl groups from acetylated xanthan pentasaccharides (Extended Data 8). The tetrasaccharide produced by the PL8 was processed by the GH88 and both GH38s, which were able to saccharify the resulting trisaccharide (Extended Data 8). The GH94 catalyzed the phosphorolysis of cellobiose in phosphate buffer, completing the full saccharification of XG (Extended Data 8). Apparent redundancy of several enzymes (CEs and GH38s) could be partially explained by different cell location (e.g. CE-A has an SPI signal while CE-B does not), unique specificities for oligosaccharide variants in size or modification (i.e. acetylation or pyruvylation), additional polysaccharides that the locus targets, or evolutionary hypotheses where this locus is in the process of streamlining or expanding. Additional support for the involvement of this locus in XG degradation is provided by RNA-seq based whole genome transcriptome analysis, which showed the induction of genes in this cluster when the community was grown on XG compared to another polysaccharide (polygalacturonic acid, PGA) that also supports R. UCG13 abundance (Extended Data 9).

\section{B. intestinalis cross-feeds on XG oligosaccharides with its xanthan utilization PUL}

Although R. UCG13 was recalcitrant to culturing efforts, we isolated several bacteria

273 hypothesized that it may be equipped to utilize smaller XG fragments, such as those released

274 during growth by R. UCG13 via its GH5 enzyme. Using the recombinant $R u$ GH5a, we generated

275 sufficient quantities of mixed XG oligosaccharides (XGOs; primarily pentameric, but also some

276 decameric oligosaccharides) to test growth of Bacteroides intestinalis. While isolates of $P$.

277 distasonis and B. clarus from the same culture showed little or no growth (Extended Data 9), 
stoichiometric mixture of the monosaccharides that compose $\mathrm{XG}$, suggesting that it utilizes most or all of the sugars contained in the XGOs (Figure 4a). Consistent with the candidate $B$. intestinalis XGOs PUL being involved in this phenotype, all of the genes in this locus were activated $>100$-fold (and some $>1000$-fold) during growth on XGOs compared to a glucosegrown reference (Figure 4b). Whole genome RNA-seq analysis of the $B$. intestinalis strain grown on XGOs revealed that the identified PUL was the most highly upregulated in the genome, further validating its role in metabolism of XGOs (Extended Data 9). Interestingly, $R u \mathrm{GH} 5 \mathrm{a}$ XGOs treated with PL8 continued to support B. intestinalis growth, but tetramer generated from the P. nanensis GH9 and PL8 failed to support any growth (Extended Data 9). Growth was rescued in the presence of glucose but not in the presence of $R u$ GH5a XGOs to upregulate the PUL (Extended Data 9), suggesting that either the B. intestinalis transporters or enzymes are incapable of processing this isomeric substrate.

To further test the role of the identified B. intestinalis PUL in XGOs degradation, we tested recombinant forms of its constituent enzymes for their ability to degrade XGOs, and confirmed the activity of several enzymes. The carbohydrate esterase domain C-terminal to the PL-CE bimodular protein was able to remove acetyl groups from acetylated xanthan pentasaccharides (Extended Data 8). While we were unable to detect xanthan lyase activity for the PL-CE enzyme on full length XG or oligosaccharides it is likely that this enzyme or another lyase acts to remove the terminal mannose residue since the GH88 was able to remove the corresponding 4,5 unsaturated glucuronic acid residue from the corresponding tetrasaccharide that would be generated by its action (Extended Data 8). The GH92 was active on the trisaccharide produced by the GH88 as observed by loss of the trisaccharide and formation of cellobiose (Extended Data 8). Finally, the GH3 was active on cellobiose, but did not show activity on either tri- or tetra- saccharide, suggesting that this enzyme may be the final step in $B$. intestinalis saccharification of XGOs (Extended Data 8). Signal peptidase II (SPII) secretion signals were predicted for the GH5, GH3, GH88, and SusD proteins while the GH92, PL-CE, HTCS, and SusC all had SPI motifs ${ }^{22}$. While signal peptides do not definitively determine cellular location, these predictions and accumulated knowledge of Sus-type systems in Bacteroidetes suggest a "selfish" model in which saccharification occurs primarily in the periplasm $^{23,37}$ (Extended Data 1). 
To determine if the original consortium was representative of all our XG-degrading cultures, we performed metagenomic sequencing on 20 additional XG-degrading communities and retrieved 16 high-quality and 3 low-quality R. UCG13 MAGs as well as an unbinned contig

314 affiliated with R. UCG13 (Supplemental Table 2). We found that the R. UCG13 XG utilization locus is extremely well conserved across these cultures with only one variation in gene content, the insertion of a GH125 coding gene, and $>95 \%$ amino acid identity (Extended Data 10). The additional GH125 gene was observed in most loci (14/17), suggesting that this gene provides a complementary, but non-essential function, possibly as an accessory $\alpha$-mannosidase $\mathrm{e}^{38}$. In contrast, only a subset of the samples (4/17) contained the B. intestinalis XGOs PUL, which showed essentially complete conservation in cultures that contained this PUL (Extended Data 10). Across all these cultures, R. UCG13 accounted for an average of only $23.1 \% \pm 1.2$ (SEM) of the total culture (Figure 1d), suggesting that additional microbes beyond $B$. intestinalis may cross-feed on products released by R. UCG13, either from degradation products of XG or by communities in samples 1, 22, and 59 contain other microbes belonging to the Bacteroidaceae family that harbor a PUL with a GH88, GH92, and GH3, suggesting that these bacteria can potentially metabolize XG-derived tetramers (Extended Data 10).

\section{Xanthan utilization loci are widespread in modern microbiomes}

Next, we asked whether XG inclusion in the modern diet could have increased the prevalence of the R. UCG13 and B. intestinalis xanthan loci compared to other populations, such as hunter-gatherers, that are less likely to be exposed to this food additive. Using each locus as a query, we searched several publicly available fecal metagenome datasets collected from populations worldwide. All modern populations sampled displayed some presence of the R. UCG13 XG locus, with the Chinese and Japanese cohorts being the highest (up to $51 \%$ in one cohort) (Figure 5). The B. intestinalis locus was less prevalent, with two industrialized population datasets (Japan and Denmark/Spain) lacking any incidence. Where the locus was present, its prevalence ranged from 1-11\%. The three hunter-gatherer or non-industrialized populations sampled, the Yanomami, Hadza, and Burkina Faso had no detected presence of either the R. UCG13 or B. intestinalis locus. 
Although the size of the hunter-gatherer datasets is relatively small, excluding the possibility of a false negative suggests several equally intriguing hypotheses. Most obviously, inclusion of XG in the modern diet may have driven either the colonization or expansion of R. UCG13 (and to a lesser extent $B$. intestinalis) into the gut communities of numerous human populations. This is in concordance with the observations of Daly et al. who found that a set of volunteers fed xanthan gum for an extended period produced stool with increased probability and degree of xanthan degradation ${ }^{39}$. Alternatively, the modern microbiome is drastically different than that of hunter-gatherers and these differences simply correlate with the abundance of R. UCG13, rather than any causal effect of XG in the diet. Another possible hypothesis is that the microbiomes of hunter-gatherer populations can degrade $\mathrm{XG}$, but through completely different microbes and pathways, a hypothesis that could be tested by culturing microbes from huntergatherer populations.

To further probe the presence of the identified XG utilization genes in other environments, we conducted an expanded LAST search $^{40}$ of both loci in 72,491 sequenced bacterial isolates and 102,860 genome bins extracted from 13,415 public metagenomes, as well as 21,762 public metagenomes that are part of the Integrated Microbial Genomes \& Microbiomes $^{41}$ (IMG/M) database using fairly stringent thresholds of $70 \%$ alignment over the query and 90\% nucleotide identity. This search yielded 35 hits of the R. UCG13 locus in human microbiome datasets, including senior adults, children, and an infant (12-months of age, Ga0169237_00111) (Supplemental Table 4). We also found 12 hits for the B. intestinalis XGOs locus, all in human microbiome samples except for a single environmental sample from a fracking water sample from deep shales in Oklahoma, USA (81\% coverage, 99\% identity) (Extended Data 10, Supplemental Table 4). XG and other polysaccharides such as guar gum are used in oil industry processes, and genes for guar gum catabolism have previously been found in oil well associated microbial communities ${ }^{42}$. Since most samples searched were nongut-derived, this demonstrates that XG-degrading R. UCG13 and XGOs-degrading B. intestinalis are largely confined to gut samples and can be present across the human lifetime.

\section{The mouse microbiome harbors a xanthan utilization locus}

To investigate the prevalence of XG-degrading populations beyond the human gut microbiome, we used samples from a previous mouse experiment in which animals fed 5\% XG 
372 showed increased levels of short chain fatty acids propionate and butyrate, suggesting the ability

373 of members of the mouse microbiome to catabolize and ferment $\mathrm{XG}^{43}$. After culturing mouse

374 feces from this experiment on XG media and confirming its ability to depolymerize XG, we used

375 metagenomics to characterize the community structure in two samples (M1741 and M737),

376 revealing the presence of a microbial species related to R. UCG13 (AAI values between the

377 human R. UCG13 and the mouse R. UCG13 were $75.7 \%$ and $75.2 \%$ for M1741 and M737,

378 respectively) as well as a XG locus with strikingly similar genetic architecture to our previously

379 characterized human XG locus (Extended Data 10, Supplemental Table 5). Although several

380 genes are well conserved across both the human and mouse isolates, we observed significant

381 divergence in the sequences of the respective $R u$ GH5a proteins that, based on data with the

382 human locus, initiate XG depolymerization. Specifically, this divergence was more pronounced

383 in the non-catalytic and non-CBM portions of the protein suggesting that while the XG-

384 hydrolyzing functions have been maintained, other domains may be more susceptible to genetic

385 drift. As with the human $R u$ GH5a, recombinant versions of the mouse $R u$ GH5a were able to

386 hydrolyze XG (Extended Data 10) but did not show significant activity on a panel of other

387 polysaccharides. These data suggest that the R. UCG13 XG locus is more broadly present in

388 mammalian gastrointestinal microbiomes and can at least be recovered through XG-feeding.

\section{Prospectus}

Our results demonstrate the existence of a multi-phylum food chain in response to XG that appears to have driven the colonization and expansion of R. UCG13 and B. intestinalis in industrialized human microbiomes. The absence of these XG-degraders in pre-industrialized microbiomes and their variable presence across post-industrialized populations suggests that XGdriven modulation of human microbiomes may be an ongoing process. The wide range in levels of XG consumption, variable presence of XG-degrading microbes across human populations, and our finding that R. UCG13 can colonize infants at an early age highlight the profound impacts that XG may be having on the assembly, stability, and evolution of industrialized human microbiomes.

The discovery of XG loci in an environmental sample and mouse microbiome, raises ecological questions about the transfer and evolution of XG utilization between host and nonhost associated environments. Although the mouse microbiome with a XG locus could have been 
exposed to XG through herbivory of $X$. campestris infected plants, mice are affiliated with human activities as pests and XG is used as a food additive in various domesticated animal foodstuffs (e.g. in calf milk replacers ${ }^{44}$ ), further solidifying a link between these loci and human activities. Since XG is a naturally biosynthesized exopolysaccharide, it is also intriguing to speculate about the role of R. UCG13's XG locus with respect to exopolysaccharides that other microbes may be producing locally in the gut.

While many questions remain about the ecological, functional, and health-relevant impacts of XG on the human microbiome, our study provides strong evidence that food additives should not be considered inert and can be drivers of microbiome ecology with potentially broad impacts.

\section{Acknowledgments}

We gratefully acknowledge Stephanie Theide for growth curve analysis suggestions and Tina Johannessen and Alexsander Lysberg for help with Nanopore metagenomics. We thank the University of Michigan Proteomics Resource Facility, Microbiome Core, and Natural Products Discovery Core for their support in completion of this project. We are grateful for support from the US National Institutes of Health (DK118024, DK125445 to ECM and UL1TR002240 in support of MPO) and the Research Council of Norway (FRIPRO program, PBP and SLLR: 250479, LHH: 302639). We thank the University of Michigan Center for Gastrointestinal Research (UMCGR), (NIDDK 5P30DK034933) for financial support with proteomics. The work conducted by the U.S. Department of Energy Joint Genome Institute, a DOE Office of Science User Facility, is supported under Contract No. DE-AC02-05CH11231.

\section{$\underline{\text { Methods }}$}

\section{Isolation, culture, and phylogenetic analysis of xanthan degrading cultures}

The original culture was isolated from a survey of 80 healthy adults using a bacterial culture strategy designed to enrich for members of the Gram-negative Bacteroidetes, a phylum that generally harbors numerous polysaccharide-degrading enzymes ${ }^{23}$. The original culture was the only XG-degrading culture isolated from this initial survey, likely due to its bias for Bacteroidetes. For subsequent surveys and further culturing fecal samples were collected into pre-reduced phosphate buffered saline, then transferred to an anaerobic chamber $(10 \% \mathrm{H} 2,5 \%$ 
$\mathrm{CO} 2$, and $85 \% \mathrm{~N} 2$; Coy Manufacturing, Grass Lake, MI) maintained at $37^{\circ} \mathrm{C}$. Fecal suspensions were used to inoculate cultures and passaged using partially Defined Medium (DM), which was generally prepared as a $2 \mathrm{x}$ stock then mixed 1:1 with $10 \mathrm{mg} / \mathrm{mL}$ carbon source (e.g. xanthan gum). Each L of prepared DM medium ( $\mathrm{pH}=7.2)$ contained $13.6 \mathrm{~g} \mathrm{KH} 2 \mathrm{PO} 4$ (Fisher, P284), $0.875 \mathrm{~g} \mathrm{NaCl}$ (Sigma, S7653), $1.125 \mathrm{~g}$ (NH4)2SO4 (Fisher, A702), $2 \mathrm{mg}$ each of adenine, guanine, thymine, cytosine, and uracil (Sigma, A2786, G11950, T0895, C3506, U1128, prepared together as 100x solution), $2 \mathrm{mg}$ of each of the 20 essential amino acids (prepared together as 100x solution), $1 \mathrm{mg}$ vitamin K3 (menadione, Sigma M5625), 0.4 mg FeSO4 (Sigma, 215422), 9.5 mg MgCl2 (Sigma, M8266), 8 mg CaCl2 (Sigma, C1016), 5 g Vitamin B12 (Sigma, V2876), $1 \mathrm{~g}$ L-cysteine, $1.2 \mathrm{mg}$ hematin with $31 \mathrm{mg}$ histidine (prepared together as 1,000x solution), $1 \mathrm{~mL}$ of Balch's vitamins, $1 \mathrm{~mL}$ of trace mineral solution, and $2.5 \mathrm{~g}$ beef extract (Sigma, B4888).

Each L of Balch's vitamins was prepared with $5 \mathrm{mg} p$-Aminobenzoic acid, $2 \mathrm{mg}$ folic acid (Sigma, F7876), $2 \mathrm{mg}$ biotin (Sigma, B4501), $5 \mathrm{mg}$ nicotinic acid (Sigma, N4126), $5 \mathrm{mg}$ calcium pantothenate (Sigma, P2250), $5 \mathrm{mg}$ riboflavin (Sigma, R7649), $5 \mathrm{mg}$ thiamine $\mathrm{HCl}$ (Sigma, T4625), $10 \mathrm{mg}$ pyridoxine $\mathrm{HCl}, 0.1 \mathrm{mg}$ cyanocobalamin, $5 \mathrm{mg}$ thioctic acid. Prepared Balch's vitamins adjusted to $\mathrm{pH} 7.0$, filter sterilized with $0.22 \mu \mathrm{m}$ PES filters, and stored in the dark at 4 C.

Each L of trace mineral solution was prepared with $0.5 \mathrm{~g}$ EDTA (Sigma, ED4SS), $3 \mathrm{~g}$ MgSO4*7H2O, 0.5 g MnSO4*H2O, 1 g NaCl (Sigma, S7653), 0.1 g FeSO4*7H2O (Sigma, 215422), 0.1 g CaCl2, 0.1 g ZnSO4*7H2O, 0.01 g CuSO4*5H2O, 0.01 g H3BO3 (Sigma, B6768), $0.01 \mathrm{~g} \mathrm{Na} 2 \mathrm{MoO} 4 * 2 \mathrm{H} 2 \mathrm{O}, 0.02 \mathrm{~g} \mathrm{NiCl} 2 * 6 \mathrm{H} 2 \mathrm{O}$. Prepared trace mineral solution was adjusted to $\mathrm{pH} 7.0$, filter sterilized with $0.22 \mu \mathrm{m}$ PES filters, and stored at room temperature. Samples that showed growth on xanthan gum, as evidenced by loss of viscosity and increased culture density, were subcultured 10 times by diluting an active culture 1:100 into fresh DM-XG medium. For the original culture, multiple samples were stored for gDNA extraction and analysis while for the larger sample set, samples were stored after 10 passages; samples were harvested by centrifugation, decanted, and stored at $-20 \mathrm{C}$ until further processing).

Frozen cell pellets were resuspended in $500 \mu \mathrm{L}$ Buffer A (200 mM NaCl, 200 mM Tris-HCl, 20 mM EDTA) and combined with $210 \mu \mathrm{L}$ SDS (20\% w/v, filter-sterilized), $500 \mu \mathrm{L}$ phenol:chloroform (alkaline $\mathrm{pH}$ ), and $\sim 250 \mu \mathrm{L}$ acid-washed glass beads (212-300 $\mu \mathrm{m}$; Sigma). 
Samples were bead beaten on high for 2-3 minutes with a Mini-BeadBeater-16 (Biospec Products, USA), then centrifuged at $18,000 \mathrm{~g}$ for $5 \mathrm{mins}$. The aqueous phase was recovered and mixed by inversion with $500 \mu \mathrm{L}$ of phenol:chloroform, centrifuged at 18,000 $g$ for 3 mins, and the aqueous phase was recovered again. The sample was mixed with $500 \mu \mathrm{L}$ chloroform, centrifuged, and then the aqueous phase was recovered and mixed with 0.1 volumes of $3 \mathrm{M}$ sodium acetate $(\mathrm{pH} 5.2)$ and 1 volume isopropanol. The sample was stored at $-80 \mathrm{C}$ for $\geq 30$ mins, then centrifuged at $\geq 20,000 \mathrm{~g}$ for 20 mins at $4 \mathrm{C}$. The pellet was washes with $1 \mathrm{~mL}$ room temperature $70 \%$ ethanol, centrifuged for 3 mins, decanted, and allowed to air dry before resuspension in $100 \mu \mathrm{L}$ sterile water. Resulting samples were additionally purified using the DNeasy Blood \& Tissue Kit (QIAGEN, USA).

Illumina sequencing, including PCR and library preparation, were performed by the University of Michigan Microbiome Core as described by Kozich et $\mathrm{al}^{45}$. Barcoded dual-index primers specific to the $16 \mathrm{~S}$ rRNA V4 region were used to amplify the DNA. PCR reactions consisted of 5 $\mu \mathrm{L}$ of $4 \mu \mathrm{M}$ equimolar primer set, $0.15 \mu \mathrm{L}$ of AccuPrime Taq DNA High Fidelity Polymerase, 2 $\mu \mathrm{L}$ of 10x AccuPrime PCR Buffer II (Thermo Fisher Scientific, catalog no. 12346094), $11.85 \mu \mathrm{L}$ of PCR-grade water, and $1 \mu \mathrm{L}$ of DNA template. The PCR conditions used consisted of 2 min at $95^{\circ} \mathrm{C}$, followed by 30 cycles of $95^{\circ} \mathrm{C}$ for $20 \mathrm{~s}, 55^{\circ} \mathrm{C}$ for $15 \mathrm{~s}$, and $72^{\circ} \mathrm{C}$ for $5 \mathrm{~min}$, followed by $72^{\circ} \mathrm{C}$ for $10 \mathrm{~min}$. Each reaction was normalized using the SequalPrep Normalization Plate Kit (Thermo Fisher Scientific, catalog no. A1051001), then pooled and quantified using the Kapa Biosystems Library qPCR MasterMix (ROX Low) Quantification kit for Illumina platforms (catalog no. KK4873). After confirming the size of the amplicon library using an Agilent Bioanalyzer and a high-sensitive DNA analysis kit (catlog no. 5067-4626), the amplicon library was sequenced on an Ilumina MiSeq platform using the 500 cycle MiSeq V2 Reagent kit (catalog no. MS-102-2003) according to the manufacturer's instructions with with modifications of the primer set with custom read 1/read 2 and index primers added to the reagent cartridge. The "Preparing Libraries for Sequencing on the MiSeq" (part 15039740, Rev. D) protocol was used to prepare libraries with a final load concentration of $5.5 \mathrm{pM}$, spiked with $15 \%$ PhiX to create diversity within the run.

Sequencing FASTQ files were analyzed using mothur (v.1.40.5) ${ }^{46}$ using the Silva reference database $^{11}$. OTUs with the same genus were combined and displayed using $\mathrm{R}^{47}$ with the packages reshape $2^{48}$, RColorBrewer ${ }^{49}$, and ggplot $2^{50}$. 


\section{Dilution to extinction experiment}

497 An overnight culture was serially diluted in 2x DM. Serial dilutions were split into two $50 \mathrm{~mL}$ tubes and mixed 1:1 with either $10 \mathrm{mg} / \mathrm{mL}$ xanthan gum or $10 \mathrm{mg} / \mathrm{mL}$ monosaccharide mixture ( $4 \mathrm{mg} / \mathrm{mL}$ glucose, $4 \mathrm{mg} / \mathrm{mL}$ mannose, $2 \mathrm{mg} / \mathrm{mL}$ sodium glucuronate), both of which also had 1 $\mathrm{mg} / \mathrm{mL}$ L-cysteine. Each dilution and carbon source was aliquoted to fill a full 96-well culture plate (Costar 3370) with $200 \mu \mathrm{L}$ per well. Plates were sealed with Breathe-Easy gas permeable sealing membrane for microtiter plates (Diversified Biotech, cat \#BEM-1). Microbial growth was measured at least 60 hours by monitoring OD $_{600}$ using a Synergy HT plate reader (Biotek Instruments) and BIOSTACK2WR plate handler (Biotek Instruments) ${ }^{51}$. Maximum OD for each substrate was measured for each culture. Full growth on substrates was conservatively defined as a maximum $\mathrm{OD}_{600}$ of $>0.7$. For each unique 96 well plate of substrate and dilution factor, the fraction of wells exhibiting full growth was calculated. Fractional growth was plotted against dilution factor for each substrate. Data were fit to the Hill equation by minimizing squared differences between the model and experimental values using Solver (GRG nonlinear) in Excel. For each experiment, a 50\% growth dilution factor (GDF 50) was calculated for each substrate at which half of the wells would be predicted to exhibit full growth.

\section{$512 \quad$ Metagenomic analysis}

513 Seven samples (15-mL) were collected at four time points (Extended Data 3; referred to as T1,

514 T2, T3 and T4) during growth of two biological replicates of the original XG-degrading culture.

515 Cells were harvested by centrifugation at $14,000 \times \mathrm{g}$ for $5 \mathrm{~min}$ and stored $\mathrm{a}-20^{\circ} \mathrm{C}$ until further use. A phenol:chloroform:isoamyl alcohol and chloroform extraction method was used to obtain high molecular weight DNA as previously described ${ }^{52}$. The gDNA was quantified using a Qubit $^{\mathrm{TM}}$ fluorimeter and the Quant-iT ${ }^{\mathrm{TM}}$ dsDNA BR Assay Kit (Invitrogen, USA), and the quality was assessed with a NanoDrop One instrument (Thermo Fisher Scientific, USA).

522 with the TrueSeq DNA PCR-free preparation and sequenced with paired ends $(2 \times 150 \mathrm{bp})$ on

524 bases on the 3 '-end with a Phred score lower than 20 and exclude all reads shorter than 100

525 nucleotides, followed by a quality filtering using the FASTX-Toolkit v.0.0.14 
527

528

529

530

531

532

533

534

535

536

537

538

539

540

541

542

543

544

545

546

547

548

549

550

551

552

553

554

555

556

$90 \%$ of the read length. Reads were co-assembled using metaSPAdes ${ }^{54}$ v3.10.1 with default parameters and k-mer sizes of 21,33, 55, 77 and 99. The resulting contigs were binned with MetaBAT $^{55}$ v0.26.3 in "very sensitive mode". The quality (completeness, contamination, and strain heterogeneity) of the metagenome assembled genomes (MAGs) was assessed by CheckM $^{56}$ v1.0.7 with default parameters. Contigs were submitted to the Integrated Microbial Genomes and Microbiomes system for open reading frames (ORFs) prediction and annotation ${ }^{57}$. Additionally, the resulting ORF were annotated for CAZymes using the CAZy annotation pipeline $^{58}$. This MAG collection was used as a reference database for mapping of the metatranscriptome data, as described below. Taxonomic classifications of MAGs were determined using both $\mathrm{MiGA}^{59}$ and GTDB-Tk ${ }^{60}$.

Human fecal samples (20) from a second enrichment experiment (unbiased towards the cultivation of Bacteroides) as well as two enrichments with mouse fecal samples were processed for gDNA extraction and library preparation exactly as described above. Metagenomic shotgun sequencing was conducted on two lanes of both Illumina HiSeq 4000 and Illumina HiSeq X Ten platforms (Illumina, Inc.) at the NSC (Oslo, Norway), and reads were quality trimmed, assembled and binned as described above. Open reading frames were annotated using PROKKA $^{61}$ v1.14.0 and resulting ORFs were further annotated for CAZymes using the CAZy annotation pipeline and expert human curation ${ }^{58}$. Completeness, contamination, and taxonomic classifications for each MAG were determined as described above. AAI comparison between the human R. UCG13 and the R. UCG13 found in the two mouse samples was determined using CompareM (https://github.com/dparks1134/CompareM).

Extracted DNA from a second enrichment experiment on XG using the original culture was prepared for long-reads sequencing using Oxford Nanopore Technologies (ONT) Ligation Sequencing Kit (SQK-LSK109) according to the manufacture protocol. The DNA library was sequenced with the ONT MinION Sequencer using a R9.4 flow cell. The sequencer was controlled by the MinKNOW software v3.6.5 running for 6 hours on a laptop (Lenovo ThinkPad P73 Xeon with data stored to 2Tb SSD), followed by base calling using Guppy v3.2.10 in 'fast' mode. This generated in total $3.59 \mathrm{~Gb}$ of data. The Nanopore reads were further processed using Filtlong v0.2.0 (https://github.com/rrwick/Filtlong), discarding the poorest 5\% of the read bases, and reads shorter than $1000 \mathrm{bp}$. 
557 The quality processed Nanopore long-reads were assembled using CANU ${ }^{62}$ v1.9 with the

558 parameters corOutCoverage $=10000$ corMinCoverage $=0$ corMhapSensitivity $=$ high genomeSize $=5 \mathrm{~m}$ redMemory $=32$ oeaMemory $=32$ batMemory $=200$. An initial polishing of the generated contigs were carried out using error-corrected reads from the assembly with minimap $2^{63}$ v2.17 - $x$ map-ont and Racon ${ }^{64}$ v1.4.14 with the argument --include-unpolished. The racon-polished contigs were further polished using Medaka v1.1.3

563 (https://github.com/nanoporetech/medaka), with the commands medaka_consensus --model 564 r941_min_fast_g303_model.hdf5. Finally, Minimap2 -ax sr was used to map quality processed Illumina reads to the medaka-polished contigs, followed by a final round of error correction using Racon with the argument --include-unpolished. Circular contigs were identified by linking

567 the contig identifiers in the polished assembly back to suggestCircular=yes in the initial contig header provided by CANU. These contigs were quality checked using CheckM ${ }^{56}$ v1.1.3 and $\mathrm{BUSCO}^{65} \mathrm{v}$ 4.1.4. Circular contigs likely to represent chromosomes ( $>1 \mathrm{Mbp}$ ) were further genecalled and functionally annotated using PROKKA ${ }^{61}$ v1.13 and taxonomically classified using GTDB-tk ${ }^{60}$ v1.4.0 with the classify_wf command. Barrnap v0.9

572 (https://github.com/tseemann/barrnap) was used to predict ribosomal RNA genes. Average 573 nucleotide Identity (ANI) was measured between the short-reads and long- reads MAGs using

574 FastANI ${ }^{66}$ v1.1 with default parameters. Short-reads MAGs were used as query while long-reads

575 MAGs were set as reference genomes. Short-reads MAG1 showed an Average Nucleotide 576 Identity (ANI) of 99.98\% with the long-reads ONT_Circ01, while short-reads MAG2 showed an 577 ANI of 99.99\% with the long-reads ONT_Circ02 (Supplemental Table 2). Phylogenetic analysis revealed that ONT_Circ02 encoded four complete 16S rRNA operons, three of which

579 were identical to the aforementioned R. UCG13 OTU.

\section{Temporal metatranscriptomic analysis of the original XG-degrading community.}

581 Cell pellets from $6 \mathrm{~mL}$ samples collected at T1-T4 during growth of two biological replicates of 582 the original XG-degrading culture were supplemented with RNAprotect Bacteria Reagent

583 (Qiagen, USA) following the manufacturer's instructions and kept at $-80{ }^{\circ} \mathrm{C}$ until RNA

584 extraction. mRNA extraction and purification were conducted as described in Kunath et al. ${ }^{67}$.

585 Samples were processed with the TruSeq stranded RNA sample preparation, which included the 586 production of a cDNA library, and sequenced on one lane of the Illumina HiSeq 3000 system 
(NSC, Oslo, Norway) to generate $2 \times 150$ paired-end reads. Prior to assembly, RNA reads were quality filtered with Trimmomatic ${ }^{68} \mathrm{v} 0.36$, whereby the minimum read length was required to be 100 bases and an average Phred threshold of 20 over a 10 nt window, and rRNA and tRNA were removed using SortMeRNA ${ }^{69}$ v.2.1b. Reads were pseudo-aligned against the metagenomic dataset using kallisto pseudo - pseudobam ${ }^{70}$. Of the 58089 ORFs (that encode proteins with $>60$ aa) identified from the metagenome of the original XG-degrading community, 7549 (13\%) were not found to be expressed, whereas $50540(87 \%)$ were expressed, resulting in a reliable quantification of the expression due to unique hits (reads mapping unambiguously against one unique ORF).

\section{Neutral Monosaccharide analysis}

The hot-phenol extraction method originally described by Massie \& $\mathrm{Zimm}^{71}$ and modified by $\mathrm{Nie}^{72}$ was used for collecting and purifying the polysaccharides remaining at different timepoints. Samples were heated to $65^{\circ} \mathrm{C}$ for $5 \mathrm{mins}$, combined with an equal volume of phenol, incubated at $65{ }^{\circ} \mathrm{C}$ for $10 \mathrm{mins}$, then cooled to $4{ }^{\circ} \mathrm{C}$ and centrifuged at $4{ }^{\circ} \mathrm{C}$ for $15 \mathrm{~min}$ at 12,000 $g$. The upper aqueous layer was collected and re-extracted using the same procedure, dialyzed extensively against deionized water (2000 Da cutoff), and freeze-dried. Neutral monosaccharide composition was obtained using the method described by Tuncil et al. ${ }^{73}$ Briefly, sugar alditol acetates were quantified by gas chromatography using a capillary column SP-2330 (SUPELCO, Bellefonte, PA) with the following conditions: injector volume, $2 \mu \mathrm{l}$; injector temperature, 240 ${ }^{\circ} \mathrm{C}$; detector temperature, $300{ }^{\circ} \mathrm{C}$; carrier gas (helium), velocity 1.9 meter/second; split ratio, 1:2; temperature program was $160^{\circ} \mathrm{C}$ for $6 \mathrm{~min}$, then $4{ }^{\circ} \mathrm{C} / \mathrm{min}$ to $220^{\circ} \mathrm{C}$ for $4 \mathrm{~min}$, then $3{ }^{\circ} \mathrm{C} / \mathrm{min}$ to $240{ }^{\circ} \mathrm{C}$ for $5 \mathrm{~min}$, and then $11^{\circ} \mathrm{C} / \mathrm{min}$ to $255^{\circ} \mathrm{C}$ for $5 \mathrm{~min}$.

\section{Thin Layer Chromatography for Localization of Enzyme Activity}

Overnight cultures were harvested at 13,000 $\mathrm{g}$ for 10 minutes. Supernatant fractions were prepared by vacuum filtration through $0.22 \mu \mathrm{m}$ PES filters. Cell pellet fractions were prepared by decanting supernatant, washing with phosphate buffered saline (PBS), spinning at 13,000 $\mathrm{g}$ for 3 mins, decanting, and resuspending in PBS. Intracellular fractions were prepared by taking cell pellet fractions and bead beating for $90 \mathrm{~s}$ with acid-washed glass beads (G1277, Sigma) in a Biospec Mini Beadbeater. Lysed culture fractions were prepared by directly bead beating unprocessed culture. 
617 Each culture fraction was mixed 1:1 with $5 \mathrm{mg} / \mathrm{mL}$ xanthan gum and incubated at $37 \mathrm{C}$ for 24

618 hours. Negative controls were prepared by heating culture fractions to $95 \mathrm{C}$ for 15 mins, then

619

620

621

622

623

624

625

626

627

628

629

630

631

632

633

634

635

636

637

638

639

640

641

642

643

644

645

646

647 centrifuging at 13,000 $\mathrm{g}$ for $10 \mathrm{mins}$ before the addition of xanthan gum. All reactions were halted by heating to $\geq 85 \mathrm{C}$ for $15 \mathrm{mins}$, then spun at $20,000 \mathrm{~g}$ for $15 \mathrm{mins}$ at $4 \mathrm{C}$. Supernatants were stored at $-20 \mathrm{C}$ until analysis by thin layer chromatography.

$3 \mu \mathrm{L}$ sample were spotted twice onto a 10x20 cm thin layer chromatography plate (Millipore TLC Silica gel 60, 20x20cm aluminum sheets), with intermediate drying using a Conair 1875 hairdryer. Standards included malto-oligosaccharides of varying lengths (Even: 2, 4, 6, Odd: 1, $3,5,7)$, glucuronic acid, and mannose. Standards were prepared at $10 \mathrm{mM}$ and $3 \mathrm{uL}$ of each was spotted onto the TLC plate. Plates were run in $\sim 100 \mathrm{~mL}$ of 2:1:1 butanol, acetic acid, water, dried, then run an additional time. After drying, plates were incubated in developing solution (100 mL ethyl acetate, $2 \mathrm{~g}$ diphenylamine, $2 \mathrm{~mL}$ aniline, $10 \mathrm{~mL}$ of $\sim 80 \%$ phosphoric acid, $1 \mathrm{~mL}$ of $\sim 38 \%$ hydrochloric acid) for $\sim 30$ seconds, then dried, and developed by holding over a flame until colors were observed.

\section{Proteomic analysis}

Approximately 1 L of xanthan gum culture was grown until it had completely liquified ( 2-3 days). Supernatant was collected by centrifuging at $18,000 \mathrm{~g}$ and vacuum filtering through a 0.2 $\mu \mathrm{m}$ PES filter. 4M ammonium sulfate was added to $200-400 \mathrm{~mL}$ of filtrate to a final concentration of 2.4M and incubated for 30-60 mins at RT or, for one sample, overnight at $4 \mathrm{C}$. Precipitated proteins were harvested by centrifugation at 18,000 $g$ for 30-60 mins, then resuspended in $50 \mathrm{mM}$ sodium phosphate $(\mathrm{pH}$ 7.5). Three different fractionation protocols were followed, but after every fractionation step, active fractions were identified by mixing $\sim 500 \mu \mathrm{L}$ with $10 \mathrm{mg} / \mathrm{mL}$ xanthan and incubating at $37 \mathrm{C}$ overnight; active-fractions were identified by loss of viscosity or production of xanthan oligosaccharides as visualized by TLC (method previously described).

1. Resuspended protein was filtered and applied to a HiTrapQ column, running a gradient from 0-100\% B (Buffer A: 50 mM sodium phosphate, $\mathrm{pH}$ 7.5; Buffer B: $50 \mathrm{mM}$ sodium phosphate, 1 $\mathrm{M} \mathrm{NaCl}, \mathrm{pH}$ 7.5). Active fractions were pooled and concentrated with a $10 \mathrm{kDa} \mathrm{MWCO}$ centricon and injected onto an S-200 16/60 column equilibrated in $50 \mathrm{mM}$ sodium phosphate, $200 \mathrm{mM} \mathrm{NaCl}, \mathrm{pH}$ 7.5. The earliest fractions to elute with significant A280 absorbance were also the most active fractions; these were pooled and submitted for proteomics. 
2. Resuspended protein was filtered and applied to an S-500 column equilibrated in $50 \mathrm{mM}$ sodium phosphate, $200 \mathrm{mM} \mathrm{NaCl}$, $\mathrm{pH}$ 7.5. Active fractions eluted in the middle of the separation were pooled and submitted for proteomics.

3. Resuspended protein was filtered and applied to an S-500 column equilibrated in $50 \mathrm{mM}$ sodium phosphate, $200 \mathrm{mM} \mathrm{NaCl}, \mathrm{pH}$ 7.5. Pooled fractions were applied to a $20 \mathrm{~mL}$ strong anion exchange column running a gradient from 0-100\% B (Buffer A: $50 \mathrm{mM}$ sodium phosphate, $\mathrm{pH}$ 7.5; Buffer B: $50 \mathrm{mM}$ sodium phosphate, $1 \mathrm{M} \mathrm{NaCl}$, $\mathrm{pH}$ 7.5). Active fractions were pooled and applied to a $1 \mathrm{~mL}$ weak anion exchange column (ANX) running a gradient from $0-100 \% \mathrm{~B}$ (Buffer A: 50 mM sodium phosphate, 10\% glycerol, pH 7.5; Buffer B: 50 mM sodium phosphate, $1 \mathrm{M} \mathrm{NaCl}, 10 \%$ glycerol, $\mathrm{pH}$ 7.5). Active fractions were pooled and submitted for proteomics.

Cysteines were reduced by adding $50 \mathrm{ml}$ of $10 \mathrm{mM}$ DTT and incubating at $45^{\circ} \mathrm{C}$ for $30 \mathrm{~min}$. Samples were cooled to room temperature and alkylation of cysteines was achieved by incubating with $65 \mathrm{mM}$ 2-Chloroacetamide, under darkness, for $30 \mathrm{~min}$ at room temperature. An overnight digestion with $1 \mathrm{ug}$ sequencing grade, modified trypsin was carried out at $37 \mathrm{C}$ with constant shaking in a Thermomixer. Digestion was stopped by acidification and peptides were desalted using SepPak C18 cartridges using manufacturer's protocol (Waters). Samples were completely dried using vacufuge. Resulting peptides were dissolved in $8 \mathrm{ml}$ of $0.1 \%$ formic $\operatorname{acid} / 2 \%$ acetonitrile solution and $2 \mu \mathrm{ls}$ of the peptide solution were resolved on a nano-capillary reverse phase column (Acclaim PepMap C18, 2 micron, $50 \mathrm{~cm}$, ThermoScientific) using a 0.1\% formic acid $/ 2 \%$ acetonitrile (Buffer A) and $0.1 \%$ formic acid $/ 95 \%$ acetonitrile (Buffer B) gradient at $300 \mathrm{nl} / \mathrm{min}$ over a period of $180 \mathrm{~min}(2-25 \%$ buffer B in $110 \mathrm{~min}, 25-40 \%$ in $20 \mathrm{~min}$, $40-90 \%$ in 5 min followed by holding at $90 \%$ buffer B for 10 min and requilibration with Buffer A for $30 \mathrm{~min}$ ). Eluent was directly introduced into $Q$ exactive $H F$ mass spectrometer (Thermo Scientific, San Jose CA) using an EasySpray source. MS1 scans were acquired at 60K resolution (AGC target $=3 \times 10^{6} ; \max$ IT $=50 \mathrm{~ms}$ ). Data-dependent collision induced dissociation MS/MS spectra were acquired using Top speed method (3 seconds) following each MS1 scan (NCE $\sim 28 \%$; $15 \mathrm{~K}$ resolution; AGC target $1 \times 10^{5}$; max IT $45 \mathrm{~ms}$ ).

Proteins were identified by searching the MS/MS data against a database of all proteins identified in the original culture metagenomes using Proteome Discoverer (v2.1, Thermo Scientific). Search parameters included MS1 mass tolerance of $10 \mathrm{ppm}$ and fragment tolerance 
of $0.2 \mathrm{Da}$; two missed cleavages were allowed; carbamidimethylation of cysteine was considered fixed modification and oxidation of methionine, deamidation of asparagine and glutamine were considered as potential modifications. False discovery rate (FDR) was determined using Percolator and proteins/peptides with an FDR of $\leq 1 \%$ were retained for further analysis.

\section{Plasmid Design and Protein Purification}

Plasmid constructs to produce recombinant proteins were made with a combination of synthesized DNA fragments (GenScript Biotech, Netherlands) and PCR amplicons using extracted culture gDNA as a template. In general, sequences were designed to remove Nterminal signaling peptides and to add a histidine tag for immobilized metal affinity chromatography (IMAC) (in many cases using the Lucigen MA101-Expresso-T7-Cloning-\&Expression-System). Plasmid assembly and protein sequences are described in Supplemental Table 6.

Constructs were transformed into HI-Control BL21(DE3) cells and single colonies were inoculated in $5 \mathrm{~mL}$ overnight LB cultures at $37^{\circ} \mathrm{C} .5 \mathrm{~mL}$ cultures were used to inoculate $1 \mathrm{~L}$ of Terrific Broth (TB) with selective antibiotic, grown to OD $\sim 0.8-1.1$ at $37^{\circ} \mathrm{C}$, and induced with $250 \mu \mathrm{M}$ IPTG. B. intestinalis enzymes were expressed at RT, while R. UCG13 enzymes were expressed at $18^{\circ} \mathrm{C}$ overnight. Cells were harvested by centrifugation and pellets were stored at $80^{\circ} \mathrm{C}$ until further processing. Proteins were purified using standard IMAC purification procedures employing sonication to lyse cells. R. UCG13 proteins were purified using $50 \mathrm{mM}$ sodium phosphate and $300 \mathrm{mM}$ sodium chloride at $\mathrm{pH} 7.5 ;$ B. intestinalis proteins were purified using $50 \mathrm{mM}$ Tris and $300 \mathrm{mM}$ sodium chloride at $\mathrm{pH}$ 8.0. All proteins were eluted from cobalt resin using buffer with the addition of $100 \mathrm{mM}$ imidazole, then buffer exchanged to remove imidazole using Zeba $2 \mathrm{~mL} 7 \mathrm{kDa}$ MWCO desalting columns. Protein concentrations were determined by measuring A280 and converting to molarity using calculated extinction coefficients.

\section{Characterization and isolation of Xanthan gum degradation products}

In general, pentameric xanthan oligosaccharides were produced by incubating $\geq 0.1$ $\mathrm{mg} / \mathrm{mL} R u$ GH5a with $5 \mathrm{mg} / \mathrm{mL}$ xanthan gum in PBS in approximately $1 \mathrm{~L}$ total volume. For xanthan tetrasaccharides, $\sim 0.5 \mathrm{U} / \mathrm{mL}$ of Xanthan lyase (E-XANLB, Megazyme) was included. After incubating 2-3 days at $37^{\circ} \mathrm{C}$ to allow complete liquefication, reactions were heat- 
inactivated, centrifuged at $\geq 10,000 \mathrm{~g}$ for $30 \mathrm{mins}$, and the supernatant was vacuum filtered through $0.22 \mu \mathrm{m}$ PES sterile filters. Supernatants were loaded onto a column containing $\sim 10 \mathrm{~g}$ of graphitized carbon (Supelclean ${ }^{\mathrm{TM}}$ ENVI-Carb ${ }^{\mathrm{TM}}$, 57210-U Supelco), washed extensively with water to remove salt and unbound material, then eluted in a stepwise fashion with increasing concentrations of acetonitrile. Fractions were dried, weighed, and analyzed by LC-MS and fractions that contained the most significant yield of desired products were combined. Highly pure products were obtained by reconstituting samples in 50\% water:acetonitrile and applying to a Luna ${ }^{\circledR} 5 \mu \mathrm{m}$ HILIC $200 \AA$ LC column $(250 \times 10$ mm) (00G-4450-N0, Phenomenex). A gradient was run from 90-20\% acetonitrile, with peaks determined through a combination of evaporative light scattering, UV, and post-run analytical LC-MS (Agilent qToF 6545) of resulting fractions.

NMR spectra were collected using an Agilent 600 NMR spectrometer $\left({ }^{1} \mathrm{H}: 600\right.$ $\mathrm{MHz},{ }^{13} \mathrm{C}: 150 \mathrm{MHz}$ ) equipped with a $5 \mathrm{~mm}$ DB AUTOX PFG broadband probe and a Varian NMR System console. All data analysis was performed using MestReNova NMR software. All chemical shifts were referenced to residual solvent peaks $\left[{ }^{1} \mathrm{H}\left(\mathrm{D}_{2} \mathrm{O}\right): 4.79 \mathrm{ppm}\right]$.

\section{Enzyme Reaction Analysis}

All enzyme reactions were carried out in 15-25 mM sodium phosphate buffer, 100-150 $\mathrm{mM}$ sodium chloride, and sometimes included up to $0.01 \mathrm{mg} / \mathrm{mL}$ bovine serum albumin (B9000S, NEB) to limit enzyme adsorption to pipettes and tubes. All R. UCG13 or B. intestinalis enzymes were tested at concentrations from 1-10 $\mu \mathrm{M}$. Cellobiose reactions were tested using 1 $\mathrm{mM}$ cellobiose at $\mathrm{pH} 7.5$, while all other reactions used $2.5 \mathrm{mg} / \mathrm{mL}$ pentasaccharide (produced using $R u \mathrm{GH} 5 \mathrm{a}$ ) and were carried out at $\mathrm{pH} 6.0$. Reactions were incubated overnight at $37^{\circ} \mathrm{C}$, halted by heating at $\geq 95^{\circ} \mathrm{C}$ for $5-10$ minutes, and centrifugation at $\geq 20,000 \mathrm{~g}$ for 10 mins.

Supernatants were mixed with 4 parts acetonitrile to yield an $80 \%$ acetonitrile solution, centrifuged for $10 \mathrm{mins}$ at $\geq 20,000 \mathrm{~g}$, and transferred into sample vials. $15 \mu \mathrm{L}$ of each sample was injected onto a Luna ${ }^{\circledR}$ Omega $3 \mu \mathrm{m}$ HILIC $200 \AA$ LC column (100 x 4.6 mm) (00D-4449E0, Phenomenex). An Agilent 1290 Infinity II HPLC system was used to separate the sample using solvent A (100\% water, 0.1\% formic acid) and solvent B (95\% acetonitrile, 5\% water, with $0.1 \%$ formic acid added) at a flow rate of $0.4 \mathrm{~mL} / \mathrm{min}$. Prior to injection and following each sample the column was equilibrated with $80 \%$ B. After injection, samples were eluted with a 30 
minute isocratic step at $80 \% \mathrm{~B}$, a 10 minute gradient decreasing B from $80 \%$ to $10 \%$, and a final column wash for 2 min at $10 \%$ B. Spectra were collected in negative mode using an Agilent 6545 LC/Q-TOF.

\section{Kinetics of RuGH5a (BCA Assay)}

Lyase-treated xanthan gum was generated by mixing $5 \mathrm{mg} / \mathrm{mL}$ xanthan gum with $0.5 \mathrm{U} / \mathrm{mL}$ of Bacillus sp. Xanthan lyase (E-XANLB, Megazyme) in $30 \mathrm{mM}$ potassium phosphate buffer (pH 6.5). After incubating overnight at $37^{\circ} \mathrm{C}$, an additional $0.5 \mathrm{U} / \mathrm{mL}$ of xanthan lyase was added. Both lyase-treated and native xanthan gum were dialyzed extensively against deionized water, heated in an $80^{\circ} \mathrm{C}$ water bath to inactivate the lyase, and centrifuged at $10,000 \mathrm{~g}$ for 20 mins to remove particulate. Supernatants were collected and stored at $4{ }^{\circ} \mathrm{C}$ until use.

Kinetic measurements were conducted using a slightly modified version of the low-volume bicinchoninic acid (BCA) assay for glycoside hydrolases used by Arnal et al ${ }^{74}$. Briefly, AEX and SEC purified $R u \mathrm{GH} 5 \mathrm{a}$ was diluted to a $10 \mathrm{x}$ stock of $5 \mu \mathrm{M}$ enzyme, $50 \mathrm{mM}$ sodium phosphate, $300 \mathrm{mM}$ sodium chloride, and $0.1 \mathrm{mg} / \mathrm{mL}$ bovine serum albumin, $\mathrm{pH} 7.5$. Reactions were $20 \mu \mathrm{L}$ of enzyme stock mixed with $180 \mu \mathrm{L}$ of various concentrations of xanthan gum. Negative controls were conducted with heat-inactivated enzyme stock. Timepoints were taken by quenching reactions with dilute, ice-cold, BCA working reagent. Reactions and controls were run with 4 independent replicates and compared to a glucose standard curve. Enzyme released reducing sugar was calculated by subtracting controls from reaction measurements.

\section{Growth curves of isolates on XG oligos}

Pure isolates from the xanthan culture were obtained by streaking an active culture onto a variety of agar plates including LB and brain heart infusion with the optional addition of $10 \%$ defibrinated horse blood (Colorado Serum Co.) and gentamicin $(200 \mu \mathrm{g} / \mathrm{mL})$. After passaging isolates twice on agar plates, individual colonies were picked and grown overnight in tryptoneyeast extract-glucose (TYG) broth medium, then stocked by mixing with 0.5 volumes each of TYG and molecular biology grade glycerol and storing at $-80^{\circ} \mathrm{C}$.

$\mathrm{DM}$ without beef extract $\left(\mathrm{DM}^{-\mathrm{BE}}\right)$, with the addition of a defined carbon source, was used to test isolates for growth on xanthan oligosaccharides. Some isolates (e.g. Parabacteroides distasonis) required the inclusion of $5 \mathrm{mg} / \mathrm{mL}$ beef extract (Sigma, B4888) to achieve robust growth on 
772 simple monosaccharides; in these cases, beef extract was included across all carbon conditions.

773 Unless otherwise specified, carbon sources were provided at a final concentration of $5 \mathrm{mg} / \mathrm{mL}$.

774 Isolates were grown overnight in TYG media, subcultured 1:50 into $\mathrm{DM}^{-\mathrm{BE}}$-glucose and grown

775 overnight, then subcultured 1:50 into $\mathrm{DM}^{-\mathrm{BE}}$ with either various carbon sources. Final cultures

776 were monitored for growth by measuring increase in absorbance $(600 \mathrm{~nm})$ using 96-well plates

777 as previously described.

\section{$778 \quad$ qPCR and RNA-seq on B. intestinalis and original community}

779 For qPCR, B. intestinalis was grown as before but cells were harvested by centrifugation at midexponential phase, mixed with RNA Protect (QIAGEN), and stored at $-80{ }^{\circ} \mathrm{C}$ until further processing. At collection, average $\mathrm{OD}_{600}$ values were $\sim 0.8$ and $\sim 0.6$ for glucose- and oligosaccharide-grown cultures, respectively. RNeasy mini kit buffers (QIAGEN) were used to extract total RNA, purified with RNA-binding spin columns (Epoch), treated with DNase I (NEB), and additionally purified using the RNeasy mini kit. SuperScript III reverse transcriptase and random primers (Invitrogen) were used to perform reverse transcription. Target transcript abundance in the resulting cDNA was quantified using a homemade qPCR mix as described previously $^{75}$ and gene-specific primers (Supplemental Table 7). Each $20 \mathrm{uL}$ reaction contained 1X Thermopol Reaction Buffer (NEB), 125uM dNTPs, 2.5mM MgSO4, 1 X SYBR Green I (Lonza), 500nM gene specific or 65nM 16S rRNA primer and 0.5 units Hot Start Taq in which raw values were normalized to $16 \mathrm{~S}$ rRNA values, then xanthan oligosaccharide values were compared to those from glucose to calculate fold-change in expression. For RNA-seq, total RNA was used from the B. intestinalis growths used for qPCR. For the community grown on XG or PGA, $5 \mathrm{~mL}$ cultures of DM-XG or DM-PGA were inoculated with a 1:100 dilution of a fully liquified DM-XG culture. PGA cultures were harvested at mid-log phase at $\mathrm{OD}_{600} \sim 0.85$ whereas $\mathrm{XG}$ cultures were harvested at late-log phase at OD600 1.2 to allow liquification of $\mathrm{XG}$, which was necessary to extract RNA from these cultures. As before,

798 cultures were harvested by centrifugation, mixed with RNA Protect (Qiagen) and stored at -80

$799{ }^{\circ} \mathrm{C}$ until further processing. RNA was purified as before except that multiple replicates of DM- 
using the Zymo RNA Clean and Concentrator ${ }^{\mathrm{TM}}-25$. About $90 \%$ rRNA depletion was achieved for all samples. B. intestinalis RNA was sequenced using NovaSeq and community RNA was sequenced using MiSeq. The resulting sequence data was analyzed for differentially expressed genes following a previously published protocol $^{76}$. Briefly, reads were filtered for quality using Trimmomatic v0.39 ${ }^{68}$. Reads were aligned to the each genome using BowTie2 v2.3.5.1 ${ }^{77}$. For the Bacteroides intestinalis transcriptome reads were aligned to its genome, while for the community data reads were aligned to either the $B$. intestinalis genome or the closed Ruminococcaceae UCG-13 metagenome assembled genome (MAG). Reads mapping to gene features were counted using htseq-count (release_0.11.1) ${ }^{78}$. Differential expression analysis was performed using the edgeR v3.34.0 package in R v.4.0.2 (with the aid of Rstudio v1.3.1093). The TMM method was used for library normalization ${ }^{79}$. Coverage data was visualized using Integrated Genome Viewer $(\mathrm{IGV})^{80}$.

\section{Extended metagenome analysis/comparison methodology}

Individual MAGs in each sample were searched by BlastP for the presence of proteins similar to those encoded by the XG-degrading PUL of R. UCG13 and B. intestinalis. This was done using the amino acid sequences of the proteins in the R. UCG13 and B. intestinalis PULs as the search homologs; both BlastP probes were searched against all the individual MAGs in the different samples with the default threshold e-value of 1e-5.

\section{Looking for R. UCG13 and B. intestinalis. XG Loci in Metagenomes}

Available cohorts of human gut metagenomic sequence data (National Center for Biotechnology Information projects: PRJNA422434 ${ }^{81}$, PRJEB10878 ${ }^{82}$, PRJEB12123 ${ }^{83}$, PRJEB12124 ${ }^{84}$, PRJEB15371 ${ }^{85}$, PRJEB6997 ${ }^{86}$, PRJDB3601 ${ }^{87}$, PRJNA48479 ${ }^{88}$, PRJEB4336 ${ }^{89}$, PRJEB2054 ${ }^{90}$, PRJNA392180 ${ }^{91}$, and PRJNA527208 ${ }^{92}$ ) were searched for the presence of xanthan locus nucleotide sequences from R. UCG13 $(92.7 \mathrm{~kb})$ and B. intestinalis $(17.9 \mathrm{~kb})$ using the following workflow: Each xanthan locus nucleotide sequence was used separately as a template and then magic-blast v1.5. $0^{93}$ was used to recruit raw Illumina reads from the available metagenomic datasets with an identity cutoff of $97 \%$. Next, the alignment files were used to generate a coverage map using bedtools v2.29.0 $0^{94}$ to calculate the percentage coverage of each sample against each individual reference. We considered a metagenomic data sample to be positive for a particular xanthan locus if it had at least $70 \%$ of the corresponding xanthan locus nucleotide sequence covered. 
834 The R. UCG13 locus and B. intestinalis XG locus were used as the query in a large-scale search

835

836

837

838

839

840

841

842

843

844

845

846

847

848

849

850

851

852

853

854

855

856

857

858

859

860

861

862 against the assembled scaffolds of isolates, metagenome assembled genomes (bins), and metagenomes included into the Integrated Microbial Genomes \& Microbiomes (IMG/M) comparative analysis system ${ }^{41}$. Within the LAST software package, version 1066, the 'lastal' tool was used with default thresholds to search the 2 loci against 72,491 public high-quality isolate genomes, and 102,860 bins from 13,415 public metagenomes, and 21,762 public metagenomes in IMG/M. Metagenome bins were generated using the binning analysis method described in A. Clum et $\mathrm{al}^{95}$.

Data availability. All sequencing reads have been deposited at the European Nucleotide Archive under BioProject PRJEB44146. All annotated MAGs are publicly available via Figshare (DOIs: 10.6084/m9.figshare.14494602, 10.6084/m9.figshare.14494536, 10.6084/m9.figshare.14494677, 10.6084/m9.figshare.14494683 and 10.6084/m9.figshare.14494689).

\section{REFERENCES}

1. García-Ochoa, F., Santos, V. E., Casas, J. A. \& Gómez, E. Xanthan gum: Production, recovery, and properties. Biotechnol. Adv. 18, 549-579 (2000).

2. Chassaing, B. et al. Dietary emulsifiers impact the mouse gut microbiota promoting colitis and metabolic syndrome. Nature 519, 92-96 (2015).

3. Collins, J. et al. Dietary trehalose enhances virulence of epidemic Clostridium difficile. Nature 553, 291-294 (2018).

4. Laudisi, F. et al. The Food Additive Maltodextrin Promotes Endoplasmic Reticulum Stress-Driven Mucus Depletion and Exacerbates Intestinal Inflammation. Cmgh 7, 457473 (2019).

5. Etienne-Mesmin, L. et al. Experimental models to study intestinal microbes-mucus interactions in health and disease. FEMS Microbiol. Rev. 43, 457-489 (2019).

6. Casas, J. A., Santos, V. E. \& García-Ochoa, F. Xanthan gum production under several operational conditions: Molecular structure and rheological properties. Enzyme Microb. Technol. 26, 282-291 (2000).

7. Sworn, G. Xanthan gum. in Handbook of Hydrocolloids 262, 833-853 (Elsevier, 2021). 
863

8. King, J. A. et al. Incidence of Celiac Disease Is Increasing Over Time. Am. J. Gastroenterol. 1 (2020). doi:10.14309/ajg.0000000000000523

9. Mortensen, A. et al. Re-evaluation of xanthan gum (E 415) as a food additive. EFSA J. 15, (2017).

10. Hehemann, J.-H., Kelly, A. G., Pudlo, N. A., Martens, E. C. \& Boraston, A. B. Bacteria of the human gut microbiome catabolize red seaweed glycans with carbohydrate-active enzyme updates from extrinsic microbes. Proc. Natl. Acad. Sci. 109, 19786-19791 (2012).

11. Quast, C. et al. The SILVA ribosomal RNA gene database project: Improved data processing and web-based tools. Nucleic Acids Res. 41, 590-596 (2013).

12. Goodman, A. L. et al. Extensive personal human gut microbiota culture collections characterized and manipulated in gnotobiotic mice. Proc. Natl. Acad. Sci. U. S. A. 108, 6252-6257 (2011).

13. Kim, C. C. et al. Genomic insights from Monoglobus pectinilyticus: a pectin-degrading specialist bacterium in the human colon. ISME J. 13, 1437-1456 (2019).

14. Ruijssenaars, H. J., De Bont, J. A. M. \& Hartmans, S. A pyruvated mannose-specific xanthan lyase involved in xanthan degradation by Paenibacillus alginolyticus XL-1. Appl. Environ. Microbiol. 65, 2446-2452 (1999).

15. Nankai, H., Hashimoto, W., Miki, H., Kawai, S. \& Murata, K. Microbial system for polysaccharide depolymerization: Enzymatic route for xanthan depolymerization by Bacillus sp. strain GL1. Appl. Environ. Microbiol. 65, 2520-2526 (1999).

16. Hashimoto, W., Nankai, H., Mikami, B. \& Murata, K. Crystal structure of Bacillus sp. GL1 xanthan lyase, which acts on the side chains of xanthan. J. Biol. Chem. 278, 7663$7673(2003)$.

17. Jensen, P. F. et al. Structure and Dynamics of a Promiscuous Xanthan Lyase from Paenibacillus nanensis and the Design of Variants with Increased Stability and Activity. Cell Chem. Biol. 26, 191-202.e6 (2019).

18. Aspeborg, H., Coutinho, P. M., Wang, Y., Brumer, H. \& Henrissat, B. Evolution, 
substrate specificity and subfamily classification of glycoside hydrolase family 5 (GH5). BMC Evol. Biol. 12, (2012).

19. Jongkees, S. A. K. \& Withers, S. G. Unusual enzymatic glycoside cleavage mechanisms. Acc. Chem. Res. 47, 226-235 (2014).

20. Rovira, C., Males, A., Davies, G. J. \& Williams, S. J. Mannosidase mechanism: at the intersection of conformation and catalysis. Curr. Opin. Struct. Biol. 62, 79-92 (2020).

21. Kool, M. M. et al. Characterization of an acetyl esterase from Myceliophthora thermophila C1 able to deacetylate xanthan. Carbohydr. Polym. 111, 222-229 (2014).

22. Almagro Armenteros, J. J. et al. SignalP 5.0 improves signal peptide predictions using deep neural networks. Nat. Biotechnol. 37, 420-423 (2019).

23. Grondin, J. M., Tamura, K., Déjean, G., Abbott, D. W. \& Brumer, H. Polysaccharide utilization loci: Fueling microbial communities. J. Bacteriol. 199, 1-15 (2017).

24. Pilgaard, B., Vuillemin, M., Holck, J., Wilkens, C. \& Meyer, A. S. Specificities and synergistic actions of novel PL8 and PL7 alginate lyases from the marine fungus Paradendryphiella salina. J. Fungi 7, 1-16 (2021).

25. Zhu, B. \& Yin, H. Alginate lyase: Review of major sources and classification, properties, structure-function analysis and applications. Bioengineered 6, 125-131 (2015).

26. Terrapon, N. et al. PULDB: The expanded database of Polysaccharide Utilization Loci. Nucleic Acids Res. 46, D677-D683 (2018).

27. Sun, Z., Liu, H., Wang, X., Yang, F. \& Li, X. Proteomic Analysis of the XanthanDegrading Pathway of Microbacterium sp. XT11. ACS Omega 4, 19096-19105 (2019).

28. Yang, F. et al. Novel Endotype Xanthanase from Xanthan-Degrading Microbacterium Microbacterium sp. Strain XT11. 85, 1-16 (2019).

29. Artzi, L., Bayer, E. A. \& Moraïs, S. Cellulosomes: Bacterial nanomachines for dismantling plant polysaccharides. Nat. Rev. Microbiol. 15, 83-95 (2017).

30. Guillén, D., Sánchez, S. \& Rodríguez-Sanoja, R. Carbohydrate-binding domains: Multiplicity of biological roles. Appl. Microbiol. Biotechnol. 85, 1241-1249 (2010). 
31. Mistry, J. et al. Pfam: The protein families database in 2021. Nucleic Acids Res. 49, D412-D419 (2021).

32. Ebbes, M. et al. Fold and function of the InlB B-repeat. J. Biol. Chem. 286, 15496-15506 (2011).

33. Bleymüller, W. M. et al. MET-activating residues in the B-repeat of the Listeria monocytogenes invasion protein InlB. J. Biol. Chem. 291, 25567-25577 (2016).

34. Kool, M. M., Gruppen, H., Sworn, G. \& Schols, H. A. Comparison of xanthans by the relative abundance of its six constituent repeating units. Carbohydr. Polym. 98, 914-921 (2013).

35. Moroz, O. V. et al. Structural Dynamics and Catalytic Properties of a Multi - Modular Xanthanase. ACS Catal. 8, 6021-6034 (2018).

36. Yang, F. et al. Production and purification of a novel xanthan lyase from a xanthandegrading microbacterium sp. Strain XT11. Sci. World J. 2014, (2014).

37. Glenwright, A. J. et al. Structural basis for nutrient acquisition by dominant members of the human gut microbiota. Nature 541, 407-411 (2017).

38. Gregg, K. J. et al. Analysis of a new family of widely distributed metal-independent $\alpha-$ mannosidases provides unique insight into the processing of N-linked glycans. J. Biol. Chem. 286, 15586-15596 (2011).

39. Daly, J., Tomlin, J. \& Read, N. W. The effect of feeding xanthan gum on colonic function in man: correlation with in vitro determinants of bacterial breakdown. Br. J. Nutr. 69, 897-902 (1993).

40. Kiełbasa, S. M., Wan, R., Sato, K., Horton, P. \& Frith, M. C. Adaptive seeds tame genomic sequence comparison. Genome Res. 21, 487-493 (2011).

41. Chen, I. M. A. et al. The IMG/M data management and analysis system v.6.0: New tools and advanced capabilities. Nucleic Acids Res. 49, D751-D763 (2021).

42. Liang, R. et al. Metabolic capability of a predominant Halanaerobium sp. in hydraulically fractured gas wells and its implication in pipeline corrosion. Front. Microbiol. 7, 1-10 
(2016).

945

946

947

948

949

950

951

952

953

954

955

956

957

958

959

960

961

962

963

964

965

966

967

968

969

970

43. Schnizlein, M. K., Vendrov, K. C., Edwards, S. J., Martens, E. C. \& Young, V. B. Dietary xanthan gum alters antibiotic efficacy against the murine gut microbiota and attenuates Clostridioides difficile colonization. bioRxiv 5, 1-10 (2019).

44. Katzbauer, B. Properties and applications of xanthan gum. Polym. Degrad. Stab. 59, 8184 (1998).

45. Kozich, J. J., Westcott, S. L., Baxter, N. T., Highlander, S. K. \& Schloss, P. D. Development of a dual-index sequencing strategy and curation pipeline for analyzing amplicon sequence data on the miseq illumina sequencing platform. Appl. Environ. Microbiol. 79, 5112-5120(2013).

46. Schloss, P. D. et al. Introducing mothur: Open-source, platform-independent, communitysupported software for describing and comparing microbial communities. Appl. Environ. Microbiol. 75, 7537-7541 (2009).

47. Team, R. C. R: A language and environment for statistical computing. (2020).

48. Wickham, H. Reshaping Data with the reshape Package. J. Stat. Softw. 21, 1-20 (2007).

49. Neuwirth, E. RColorBrewer: ColorBrewer Palettes. (2014). Available at: https://cran.rproject.org/package $=$ RColorBrewer.

50. Wickham, H. Elegant Graphics for Data Analysis: ggplot2. Applied Spatial Data Analysis with $R$ (2008).

51. Martens, E. C. et al. Recognition and degradation of plant cell wall polysaccharides by two human gut symbionts. PLoS Biol. 9, (2011).

52. Pope, P. B. et al. Isolation of Succinivibrionaceae implicated in low methane emissions from Tammar wallabies. Science (80-. ). 333, 646-648 (2011).

53. Martin, M. Cutadapt removes adapter sequences from high-throughput sequencing reads. EMBnet.journal 17, 10 (2011).

54. Nurk, S., Meleshko, D., Korobeynikov, A. \& Pevzner, P. A. MetaSPAdes: A new versatile metagenomic assembler. Genome Res. 27, 824-834 (2017). 
971 55. Kang, D. D., Froula, J., Egan, R. \& Wang, Z. MetaBAT, an efficient tool for accurately

972

973

974

975

976

977

978

979

980

981

982

983

984

985

986

987

988

989

990

991

992

993

994

995

996

997 reconstructing single genomes from complex microbial communities. PeerJ 2015, 1-15 (2015).

56. Parks, D. H., Imelfort, M., Skennerton, C. T., Hugenholtz, P. \& Tyson, G. W. CheckM: Assessing the quality of microbial genomes recovered from isolates, single cells, and metagenomes. Genome Res. 25, 1043-1055 (2015).

57. Chen, I. M. A. et al. IMG/M: Integrated genome and metagenome comparative data analysis system. Nucleic Acids Res. 45, D507-D516 (2017).

58. Lombard, V., Golaconda Ramulu, H., Drula, E., Coutinho, P. M. \& Henrissat, B. The carbohydrate-active enzymes database (CAZy) in 2013. Nucleic Acids Res. 42, 490-495 (2014).

59. Rodriguez-R, L. M. et al. The Microbial Genomes Atlas (MiGA) webserver: Taxonomic and gene diversity analysis of Archaea and Bacteria at the whole genome level. Nucleic Acids Res. 46, W282-W288 (2018).

60. Chaumeil, P. A., Mussig, A. J., Hugenholtz, P. \& Parks, D. H. GTDB-Tk: A toolkit to classify genomes with the genome taxonomy database. Bioinformatics 36, 1925-1927 (2020).

61. Seemann, T. Prokka: Rapid prokaryotic genome annotation. Bioinformatics 30, 20682069 (2014).

62. Koren, S. et al. Canu: scalable and accurate long-read assembly via adaptive k -mer weighting and repeat separation. Genome Res. 27, 722-736 (2017).

63. Li, H. Minimap2: Pairwise alignment for nucleotide sequences. Bioinformatics 34, 30943100 (2018).

64. Vaser, R., Sović, I., Nagarajan, N. \& Šikić, M. Fast and accurate de novo genome assembly from long uncorrected reads. Genome Res. 27, 737-746 (2017).

65. Seppey, M., Manni, M. \& Zdobnov, E. M. BUSCO: Assessing Genome Assembly and Annotation Completeness BT - Gene Prediction: Methods and Protocols. (2019). 
66. Jain, C., Rodriguez-R, L. M., Phillippy, A. M., Konstantinidis, K. T. \& Aluru, S. High throughput ANI analysis of 90K prokaryotic genomes reveals clear species boundaries. Nat. Commun. 9, 1-8 (2018).

67. Kunath, B. J. et al. From proteins to polysaccharides: lifestyle and genetic evolution of Coprothermobacter proteolyticus. ISME J. 13, 603-617 (2019).

68. Bolger, A. M., Lohse, M. \& Usadel, B. Trimmomatic: A flexible trimmer for Illumina sequence data. Bioinformatics 30, 2114-2120 (2014).

69. Kopylova, E., Noé, L. \& Touzet, H. SortMeRNA: Fast and accurate filtering of ribosomal RNAs in metatranscriptomic data. Bioinformatics 28, 3211-3217 (2012).

70. Bray, N. L., Pimentel, H., Melsted, P. \& Pachter, L. Near-optimal probabilistic RNA-seq quantification. Nat. Biotechnol. 34, 525-527 (2016).

71. Massie, H. R. \& Zimm, B. H. THE USE OF HOT PHENOL IN PREPARING DNA. Proc. Natl. Acad. Sci. 54, 1641-1643 (1965).

72. Nie, X. Relationships between dietary fiber structural features and growth and utilization patterns of human gut bacteria. ProQuest Diss. Theses 136 (2016).

73. Tuncil, Y. E., Thakkar, R. D., Marcia, A. D. R., Hamaker, B. R. \& Lindemann, S. R. Divergent short-chain fatty acid production and succession of colonic microbiota arise in fermentation of variously-sized wheat bran fractions. Sci. Rep. 8, 1-13 (2018).

74. Arnal, G., Attia, M. A., Asohan, J. \& Brumer, H. A Low-Volume, Parallel CopperBicinchoninic Acid (BCA) Assay for Glycoside Hydrolases. in Protein-Carbohydrate Interactions. Methods and Protocols (eds. Abbott, D. W. \& Lammerts van Bueren, A.) 1588, 209-214 (Springer New York, 2017).

75. Speer, M. A. DEVELOPMENT OF A GENETICALLY MODIFIED SILAGE INOCULANT FOR THE BIOLOGICAL PRETREATMENT OF LIGNOCELLULOSIC BIOMASS. (Pennsylvania State University, 2013).

76. Anders, S. et al. Count-based differential expression analysis of RNA sequencing data using R and Bioconductor. Nat. Protoc. 8, 1765-1786 (2013). 
77. Langmead, B. \& Salzberg, S. L. Fast gapped-read alignment with Bowtie 2. Nat. Methods 9, 357-359 (2012).

78. Anders, S., Pyl, P. T. \& Huber, W. HTSeq-A Python framework to work with highthroughput sequencing data. Bioinformatics 31, 166-169 (2015).

79. Robinson, M. D., McCarthy, D. J. \& Smyth, G. K. edgeR: A Bioconductor package for differential expression analysis of digital gene expression data. Bioinformatics 26, 139140 (2009).

80. Thorvaldsdóttir, H., Robinson, J. T. \& Mesirov, J. P. Integrative Genomics Viewer (IGV): High-performance genomics data visualization and exploration. Brief. Bioinform. 14, 178192 (2013).

81. Wang, J. et al. A metagenome-wide association study of gut microbiota in type 2 diabetes. Nature 490, 55-60 (2012).

82. Yu, J. et al. Metagenomic analysis of faecal microbiome as a tool towards targeted noninvasive biomarkers for colorectal cancer. Gut 66, 70-78 (2017).

83. Liu, R. et al. Gut microbiome and serum metabolome alterations in obesity and after weight-loss intervention. Nat. Med. 23, 859-868 (2017).

84. Gu, Y. et al. Analyses of gut microbiota and plasma bile acids enable stratification of patients for antidiabetic treatment. Nat. Commun. 8, (2017).

85. He, Q. et al. Two distinct metacommunities characterize the gut microbiota in Crohn's disease patients. Gigascience 6, 1-11 (2017).

86. Zhang, X. et al. The oral and gut microbiomes are perturbed in rheumatoid arthritis and partly normalized after treatment. Nat. Med. 21, 895-905 (2015).

87. Nishijima, S. et al. The gut microbiome of healthy Japanese and its microbial and functional uniqueness. DNA Res. 23, 125-133 (2016).

88. Lloyd-Price, J. et al. Strains, functions and dynamics in the expanded Human Microbiome Project. Nature 550, 61-66 (2017).

89. Le Chatelier, E. et al. Richness of human gut microbiome correlates with metabolic 
markers. Nature 500, 541-546 (2013).

1053

1054

1055

1056

1057

1058

1059

1060

1061

1062

1063

1064

1065

1066

1067

1068

1069

1070

1071

1072

1073

1074

1075

1076

1077

1078

1079

90. Qin, J. et al. A human gut microbial gene catalogue established by metagenomic sequencing. Nature 464, 59-65 (2010).

91. Smits, S. A. et al. Seasonal cycling in the gut microbiome of the Hadza hunter-gatherers of Tanzania. Science (80-. ). 357, 802-805 (2017).

92. Conteville, L. C., Oliveira-Ferreira, J. \& Vicente, A. C. P. Gut microbiome biomarkers and functional diversity within an Amazonian semi-nomadic hunter-gatherer group. Front. Microbiol. 10, 1-10 (2019).

93. Boratyn, G. M., Thierry-Mieg, J., Thierry-Mieg, D., Busby, B. \& Madden, T. L. MagicBLAST, an accurate RNA-seq aligner for long and short reads. BMC Bioinformatics 20, $1-19$ (2019).

94. Quinlan, A. R. \& Hall, I. M. BEDTools: A flexible suite of utilities for comparing genomic features. Bioinformatics 26, 841-842 (2010).

95. Clum, A. et al. The DOE JGI Metagenome Workflow. bioRxiv (2020). doi:https://doi.org/10.1101/2020.09.30.320929

\section{FIGURE LEGENDS}

Figure 1. R. UCG13 was a common factor across xanthan gum degrading cultures.

a, Xanthan gum is a repeating structure of glucose (blue circles), mannose (green circles), and glucuronic acid (blue and white diamond). The inner and outer mannose residues are variably modified by acetylation and pyruvylation, respectively.

b, Growth curves of the original xanthan-degrading culture showed greater culture density as xanthan gum concentration was increased $(n=12, S E M \leq 3 \%)$, and, $\mathbf{c}$, displayed relatively stable composition over sequential passaging. Passaging the culture on BHI-blood plates resulted in a loss of R. UCG13 as well as xanthan degrading activity.

d, An additional 20 samples were sequentially passaged in xanthan containing media (10x) and analyzed for composition by $16 \mathrm{~S}$ rRNA sequencing (16 of the most abundant genus are displayed for clarity). All cultures shared an abundant OTU, classified as R. UCG13. 
1081 Figure 2. Metagenomics, metatranscriptomics, and activity-guided proteomics identified two 1082 putative xanthan gum degrading loci.

1083 Putative xanthan utilization loci color-coded and annotated by predicted protein family. The four

1084 boxes below each gene are colored to represent expression levels of each gene at timepoints

1085 taken throughout the culture's growth on xanthan gum. MAG taxonomy is indicated in

1086 parentheticals.

Figure 3. R. UCG13 encodes a novel GH5 that depolymerizes native xanthan gum.

a, Extracted ion chromatograms showing various acetylated and pyruvylated penta- and decasaccharides produced by incubating culture supernatant with XG.

b, Annotated domains of the xanthan-degrading $R u$ GH5a, showing its signal peptide (SP), three carbohydrate binding modules (CBMs), and multiple Listeria-Bacteroides repeat domains. xanthan gum with either $R u$ GH5a or P. nanensis GH9. standard deviation, $\mathrm{n}=4$ )

Figure 4. B. intestinalis cross-feeds on xanthan oligosaccharides.

a, Growth curves of $B$. intestinalis isolated from the original xanthan-degrading culture. (curves represent mean and SEM, $\mathrm{n}=2$ ).

1101 b, Fold-change in expression of B. intestinalis genes when grown on xanthan oligosaccharides 1102 relative to glucose.

Figure 5. Xanthan degrading loci are present in modern human microbiomes but not in hunter1106 gatherers'.

1107 Multiple microbiome metagenome datasets were searched for the presence or absence of the R.

1108 UCG13 and B. intestinalis xanthan loci. Map colors correspond to where populations were 1109 sampled for each dataset displayed on the outside of the figure. Circle segments are sized proportionately to total number of individuals sampled for each dataset. Lines represent presence 
1111 of either the R. UCG13 xanthan locus (green) or the B. intestinalis xanthan locus (red).

1112 Percentages display the total abundance of R. UCG13 or B. intestinalis locus in each dataset.

1114 Extended Data 1. Cellular model of xanthan degradation

Extended Data 2. Xanthan degradation is a multi-species phenotype.

1117 An active xanthan culture was diluted in 2x defined media without a major carbon source, then

1118 divided and diluted 1:1 with either $2 x$ xanthan gum or $2 x$ monosaccharide mix (2:2:1

1119 mannose;glucose;glucuronic acid), then aliquoted into $200 \mathrm{uL}$ cultures in 96-well plates. Each

1120 datapoint represents the fraction of cultures (out of 96) growing above OD600 0.7 at each

1121 dilution, grown in either xanthan gum or monosaccharide mix media. Data were fit to the Hill

1122 equation to calculate a 50\% growth dilution factor (GDF 50) at which half of the cultures would

1123 grow above OD 0.7. Across 5 independent experiments, there was a GDF50 difference of 1.8

1124 (standard deviation $=0.4, \mathrm{SEM}=0.2$ ). This demonstrates that at comparable dilutions, microbes

1125 were present that could grow on monosaccharides but were unable to grow using XG, suggesting

1126 that several microbes are required in this media to allow growth on XG.

1128 Extended Data 3. Neutral monosaccharide and metatranscriptomic analysis

1129 a, Two replicates of the original xanthan culture were grown and sampled at multiple timepoints

1130 for $\mathbf{b}$, neutral monosaccharide analysis of residual xanthan gum ( $\mathrm{n}=3$, standard deviation shown)

1131 and c, metatranscriptomic analysis of annotated CAZymes in each of the MAGs (completeness

1132 value $>75 \%$ ) reconstructed from metagenomic data from the enrichment culture. MAG

1133 taxonomy (Supplementary Table 2) is indicated in parentheticals. An "\#” indicates a low

1134 AAI $\%$.

1136 Extended Data 4. Culture supernatant contains enzymes capable of depolymerizing xanthan

1137 gum, while intracellular contents are required for complete saccharification.

1138 Thin layer chromatography of xanthan gum incubated with different fractions of an active

1139 xanthan gum culture (supernatant, washed cell pellet, lysed cell pellet, or lysed culture).

1140 Negative controls were prepared by heating fractions at $95 \mathrm{C}$ for 15 minutes prior to initiating

1141 with xanthan gum. EDTA was added to a final concentration of $\sim 50 \mathrm{mM}$ to determine the 
necessity of divalent cations for enzyme activity. Strong color development in circles at baseline is undigested polysaccharide while bands that migrated with solvent are digested oligosaccharides and monosaccharides.

Extended Data 5. Activity-guided fractionation and proteomics narrow list of potential xanthanases.

a, Venn diagram depicting activity-guided fractionation of culture supernatants followed by proteomic identification of candidate proteins present across all active preparations.

b, SDS-PAGE of one of the fractionated culture supernatants (ANX processed sample) submitted for proteomic analysis (without and with boiling prior to analysis). Protein complexes or fragments that are larger are retained at the top of the gel while smaller proteins migrate towards the bottom of the gel. The ladder on the left shows how 180, 115, and $82 \mathrm{kDa}$ standards are retained by the gel matrix. Boiling results in separation of protein complexes that cause streaking in the first lane and resolution into single protein bands that are denatured and migrate with respect to size.

c, Proteomics narrows potential xanthanases to 33 proteins, 22 of which were from R. UCG13. Final candidates were obtained by collating proteins that were present across all three activitybased fractionation experiments with proteomic identification. Colors assist with visualizing number of peptides associated with each protein at different thresholds $(<5$, red; 5-24, orange; $25-74$, blue; $\geq 75$, green)

\section{Extended Data 6. Activity of R. UCG13 GH5 enzymes on various polysaccharides.}

a, SDS-PAGE gel of purified GH5 constructs and their resultant activity as assessed by TLC on b, xanthan gum, b-c, carboxymethyl cellulose (CMC), c, hydroxyethyl cellulose (HEC), d, barley $\beta$-glucan, $\mathbf{d - e}$, yeast $\beta$-glucan, $\mathbf{e}$, tamarind xyloglucan, $\mathbf{f}$, xylan, and $\mathbf{f}$-g, wheat arabinoxylan. Enzymes are 1, RuGH5b (GH5 only); 2, RuGH5b (GH5 with CBM-A); 3, $R u \mathrm{GH} 5 \mathrm{~b}$ (GH5 with CBM-A/B); 4, RuGH5b (full protein); 5, RuGH5a (GH5 only); 6, RuGH5a (GH5 with CBM-A); 7, RuGH5a (GH5 with CBM-A/B); 8, RuGH5a (GH5 with CBM-A/B/C); 9, $R u$ GH5a (full protein); 10, replicate of 8 . Strong color development in circles at baseline is undigested polysaccharide while bands or streaking that migrated with solvent are digested oligosaccharides and monosaccharides. Although minor streaking appears in some substrates due 
1173 to residual oligosaccharides, comparing untreated substrate with enzyme incubated substrate

1174 allows determination of enzyme activity. RuGH5a constructs with all 3 CBMs (8-10) showed

1175 clear activity on XG.

1176

1177 Extended Data 7. NMR of tetrasaccharide produced by $R u$ GH5a (and PL8 xanthan lyase).

1178 a,,${ }^{13} \mathrm{C}-\mathrm{NMR}$ spectrum of tetrasaccharide in $\mathrm{D}_{2} \mathrm{O}\left({ }^{13} \mathrm{C}: 150 \mathrm{MHz}\right)$.

1179 b, COSY $\left({ }^{1} \mathrm{H}-{ }^{1} \mathrm{H}\right)$ spectrum of tetrasaccharide in $\mathrm{D}_{2} \mathrm{O}(600 \mathrm{MHz})$.

1180 c, HSQC $\left({ }^{1} \mathrm{H}^{-13} \mathrm{C}\right)$ spectrum of tetrasaccharide in $\mathrm{D}_{2} \mathrm{O}$. Red contours represent $\mathrm{CH}$ and $\mathrm{CH}_{3}$

1181 groups, blue contours represent $\mathrm{CH}_{2}$ groups.

1182 d, $\mathrm{HMBC}\left({ }^{1} \mathrm{H}^{-13} \mathrm{C}\right)$ spectrum of tetrasaccharide in $\mathrm{D}_{2} \mathrm{O}$.

1183 e, Selective 1H 1D-TOCSY spectrum of tetrasaccharide in $\mathrm{D}_{2} \mathrm{O}\left({ }^{1} \mathrm{H}: 600 \mathrm{MHz}\right)$. Starred peak

1184 indicates the frequency irradiated $(5.83 \mathrm{ppm})$ and arrow on the structure illustrates corresponding

1185 proton position being irradiated $(\Delta 4,5-\mathrm{GlcA} H 5)$.

1186 f, Selective 1H 1D-TOCSY spectrum of tetrasaccharide in $\mathrm{D}_{2} \mathrm{O}\left({ }^{1} \mathrm{H}: 600 \mathrm{MHz}\right)$. Starred peak

1187 indicates the frequency irradiated $(5.35 \mathrm{ppm})$ and arrow on the structure illustrates corresponding

1188 proton position being irradiated (Man H1).

1189 g, Selective 1H 1D-TOCSY spectrum of tetrasaccharide in $\mathrm{D}_{2} \mathrm{O}\left({ }^{1} \mathrm{H}: 600 \mathrm{MHz}\right)$. Starred peak

1190 indicates the frequency irradiated $(4.27 \mathrm{ppm})$ and arrow on the structure illustrates corresponding

1191 proton position being irradiated (Man H2).

$1192 \mathbf{h}$, Selective 1H 1D-TOCSY spectrum of tetrasaccharide in $\mathrm{D}_{2} \mathrm{O}\left({ }^{1} \mathrm{H}: 600 \mathrm{MHz}\right)$. Starred peak

1193 indicates the frequency irradiated $(4.52 \mathrm{ppm})$ and arrow on the structure illustrates corresponding

1194 proton position being irradiated (Glc H1).

1195 i, Selective 1H 1D-TOCSY spectrum of tetrasaccharide in $\mathrm{D}_{2} \mathrm{O}\left({ }^{1} \mathrm{H}: 600 \mathrm{MHz}\right)$. Starred peak

1196 indicates the frequency irradiated $(4.64 \mathrm{ppm})$ and arrow on the structure illustrates corresponding

1197 proton position being irradiated ( $\beta$-Glc H1).

$1198 \mathbf{j}$, Selective 1H 1D-TOCSY spectrum of tetrasaccharide in $\mathrm{D}_{2} \mathrm{O}\left({ }^{1} \mathrm{H}: 600 \mathrm{MHz}\right)$. Starred peak

1199 indicates the frequency irradiated $(5.20 \mathrm{ppm})$ and arrow on the structure illustrates corresponding

1200 proton position being irradiated ( $\alpha$-Glc H1).

1201

$1202{ }^{1} \mathrm{H}-\mathrm{NMR}$ analysis illustrated an inconsistent spectrum to that of the known degradation product 1203 from other xanthanases (including GH9 from Paenibacillus nanensis or the $\beta$-D-glucanase in 
Bacillus sp. Strain GL1), that hydrolyze xanthan at the reducing end of the branching glucose. LCMS analysis was consistent with a tetrasaccharide containing a $\Delta 4,5$-ene-GlcA moiety, but despite the appropriate mass, these differences in the ${ }^{1} \mathrm{H}-\mathrm{NMR}$ suggested an alternative cut site. To confirm, full structural elucidation was conducted by extensive NMR-analysis. In a similar fashion to Wilson and coworkers, spin systems for each monosaccharide were established via selective 1D-TOCSY experiments, selectively irradiating individual anomeric protons between $\delta_{\mathrm{H}} 4.52$ and $\delta_{\mathrm{H}} 5.35$, and the one vinylic proton of the $\Delta 4,5$-ene-GlcA residue at $\delta_{\mathrm{H}} 5.83$. This vinylic proton was easily identified by HSQC analysis via its distinct ${ }^{1} J_{\mathrm{H}, \mathrm{C}}$ correlation $\left(\delta_{\mathrm{C}} 107.9\right.$ $\mathrm{ppm})$, and its $\mathrm{HMBC}$ correlations to C-5 ( $\left.\delta_{\mathrm{C}} 144.4 \mathrm{ppm}\right)$ and C-6 $\left(\delta_{C} 168.8 \mathrm{ppm}\right)$ of $\Delta 4,5$-eneGlcA. This proton was used as a starting point for structural elucidation, and in conjunction with the data obtained from 2D-HSQC and selective 1D-TOCSY experiments allowed for identification of the remainder of the $\Delta 4,5$-ene-GlcA spin system, including the anomeric position $\left(\delta_{\mathrm{H}} 5.06, \delta_{\mathrm{C}} 99.2\right)$. Further HMBC analysis identified a correlation between $\mathrm{H}-1$ of $\Delta 4,5-$ ene-GlcA and the $\mathrm{C}-2$ position of $\operatorname{Man}\left(\delta_{\mathrm{C}} 76.4 \mathrm{ppm}\right)$. The inverse $\mathrm{HMBC}$ correlation was also observed from H-2 of the Man residue ( $\left.\delta_{\mathrm{H}} 4.27\right)$ to the anomeric carbon of $\Delta 4,5$-ene-GlcA ( $\delta_{\mathrm{C}}$ 99.2), confirming the expected connectivity through a $1 \rightarrow 2$ linkage. COSY analysis identified a correlation between $\mathrm{H}-2$ and $\mathrm{H}-1\left(\delta_{\mathrm{H}} 5.35\right)$ of the of the $\alpha$-Man residue, and as was the case for $\Delta 4,5$-ene-GlcA, the remainder of the positions were assigned from 2D-HSQC and selective 1DTOCSY experiments. Interestingly, the anomeric position of $\alpha$-Man appeared as a singlet, in contrast to the reported two anomeric proton signals associated with this residue in the tetrasaccharide isolated by Wilson. HMBC analysis from this anomeric position showed correlations to C-2, C-3, and C-5 $\left(\delta_{\mathrm{C}} 76.4, \delta_{\mathrm{C}} 69.4\right.$, and $\delta_{\mathrm{C}} 72.5$ respectively) of Man. An additional correlation was observed to a carbon external to the Man subunit, with a chemical shift of $82.5 \mathrm{ppm}$. This shift was identified as belonging to the $\mathrm{C}-3$ position of a nonreducing glucosyl residue. This was confirmed via HMBC correlations from both H-2 and H-4 of Glc(n.r). The H-2 peak was free of any overlap in the ${ }^{1} \mathrm{H}-\mathrm{NMR}$ spectrum, allowing for unambiguous assignment through a COSY correlation between itself and $\mathrm{H}-1\left(\delta_{\mathrm{H}} 3.37\right.$ and $\delta_{\mathrm{H}} 4.52$ respectively), as well as the $\mathrm{H}-3$ proton with a chemical shift of $3.63 \mathrm{ppm}$. This gave us confidence that the $\alpha$-Man residue was connected via a $1 \rightarrow 3$ linkage to this Glc(n.r) subunit. Importantly, the anomeric proton of the Glc(n.r) residue appeared as a single doublet, integrating with a value of one in the ${ }^{1} \mathrm{H}-\mathrm{NMR}$ spectrum with a coupling constant of $8.0 \mathrm{~Hz}$, consistent with 
1235 a $\beta$-configured Glc(n.r) monomer. This confirmed connectivity between the $\alpha$-Man and $\beta$ -

1236 Glc(n.r) residues, suggesting a disparate structure to the previously reported degradation product.

1237 Finally, a key HMBC correlation was observed from the anomeric proton $\left(\delta_{\mathrm{H}} 4.52\right)$ to an external

1238 carbon with a ${ }^{13} \mathrm{C}$-chemical shift of $\sim 79 \mathrm{ppm}$. Upon closer inspection, this carbon was actually

1239 two separate peaks, corresponding to the C-4 position of the alpha (minor) and beta (major)

1240 anomers $\left(\delta_{\mathrm{C}} 79.0\right.$ and $\delta_{\mathrm{C}} 78.8$ respectively) of the reducing Glc. This confirmed the expected $1 \rightarrow$

12414 linkage of the backbone Glc residues, albeit illustrating hydrolysis had occurred at the reducing

1242 end of the nonbranching Glc. To complete structural elucidation, the remainder of the positions

1243 were assigned from 2D-HSQC and selective 1D-TOCSY experiments for both the alpha and beta

1244 anomers separately.

1245

1246 Extended Data 8. Activity of R. UCG13 and B. intestinalis enzymes.

1247 LC-MS analysis was used to track relative increases and decreases of intermediate

1248 oligosaccharides with the addition of enzymes, verifying their abilities to successively cleave XG

1249 pentasaccharides to their substituent monosaccharides. Integrated extracted ion counts $(\mathrm{n}=4$,

1250 SEM) that correlate with compound abundance are shown for a, acetylated pentasaccharide (M-

1251 H ions: 883.26, 953.26, 925.27), b, deacetylated pentasaccharide (M-H ions: 841.25, 911.25), c,

1252 acetylated tetrasaccharide (2M-H ion: 1407.39), d, tetrasaccharide (M-H ion: 661.18), e,

1253 acetylated trisaccharide $(\mathrm{M}+\mathrm{Cl}$ ion: 581.15), $\mathbf{f}$, trisaccharide $(\mathrm{M}+\mathrm{Cl}$ ion: 539.14), $\mathbf{g}$, cellobiose

1254 ( $\mathrm{M}+\mathrm{Cl}$ ion: 377.09), and $\mathbf{h}$, pyruvylated mannose (M-H ion: 249.06). Reactions were carried out

1255 using xanthan oligosaccharides produced by the $R u$ GH5a to test activities of the R. UCG13 (A-I)

1256 and B. intestinalis (J-O) enzymes. R. UCG13 enzymes were tested in reactions that included (A)

1257 no enzyme, (B) R. UCG13 CE-A, (C) R. UCG13 CE-B, (D) R. UCG13 PL8, (E) R. UCG13 PL8

1258 and CE-A, (F) R. UCG13 PL8 and CE-B, (G) R. UCG13 PL8, both CEs, and GH88, (H) R.

1259 UCG13 PL8, both CEs, GH88, and GH38-A, (I) R. UCG13 PL8, both CEs, GH88, and GH38-B.

1260 B. intestinalis enzymes were tested in reactions that included ( $\mathrm{J}$ ) no enzyme, $(\mathrm{K}) \mathrm{Bi}$ PL-only, (L)

1261 Bi PL-CE, (M) Bi PL-CE and Bacillus PL8, (N) Bi PL-CE and GH88 and Bacillus PL8, (O) Bi

1262 PL-CE, GH88, and GH92 and Bacillus PL8.

1263 i, Legend of enzymes included in each reaction. 
1265 Recombinant enzymes were purified and analyzed for expression and purity by SDS-PAGE.

1266 Proteins generally expressed well with a single dense band indicating overexpression of the 1267 enzyme at its predicted molecular weight as compared to a size ladder. Exceptions included the

1268 R. UCG13 GH88 and CE-A, both of which had bands at the predicted enzyme size but also

1269 showed bands of comparable density at other sizes resulting from either proteolysis or co1270 purification of undesired E. coli proteins.

1271 j, SDS-PAGE gel of purified enzymes with $4.5 \mu \mathrm{g}$ loaded, including (1-2) ladder, (3) $B$.

1272 intestinalis GH3, (4) B. intestinalis GH5, (5) B. intestinalis PL-only, (6) B. intestinalis PL-CE,

1273 (7) B. intestinalis GH88, (8) B. intestinalis GH92, (9) R. UCG 13 GH38-A, (10) R. UCG13

1274 GH38-B, (11) R. UCG13 GH94, (12) R. UCG13 PL8, (13) R. UCG13 CE-A.

1275

1276

$\mathbf{k}$, SDS-PAGE gel of purified enzymes with $4.5 \mu \mathrm{g}$ loaded, including (1) ladder, (2) $B$.

1277 intestinalis PL-only, (3) B. intestinalis PL-CE, (4) B. intestinalis GH88, (5) B. intestinalis GH92,

1278 (6) R. UCG13 GH38-A, (7) R. UCG13 GH38-B, (8) R. UCG13 CE-A, (9) R. UCG13 GH88,

1279 (10) R. UCG13 CE-B, (11) R. UCG13 PL8.

1280

1, TLC analysis showed that R. UCG13 GH94 and B. intestinalis GH3 are active on cellobiose. From left to right lane show (A) $R u \mathrm{GH} 5 \mathrm{~b}$ (full protein), (B) $R u \mathrm{GH} 5 \mathrm{a}$ (full protein), (C) B. intestinalis GH3, (D) B. intestinalis GH5, (E) R. UCG13 GH94, (F) odd standards, (G) even standards, $(\mathrm{H})$ cellobiose. Odd and even standards are maltooligosaccharides with 1, 3, 5, and 7 hexoses or 2, 4, and 6 hexoses, respectively. While the B. intestinalis GH3 only produced one product, the R. UCG13 GH94 produced two, one matching the approximate Rf of glucose

1287 while the other had a much lower Rf which presumably is phosphorylated glucose (matching the 1288 known phosphorylase activity of the GH94 family).

Extended Data 9.

1291 a, Traces of RNA-seq expression data from triplicates of the original culture grown on either XG or polygalacturonic acid (PGA), illustrating overexpression of the XG PUL. b, Bacteroides

1293 clarus and c, Parabacteroides distasonis isolated from the original culture did not grow on

1294 XGOs. d, Bacteroides intestinalis did not grow on tetramer generated with P. nanensis GH9 and 1295 PL8 (Psp Tetramer) even in the presence of $1 \mathrm{mg} / \mathrm{mL}$ RuGH5a generated XGOs to activate the 
PUL. Growth on glucose confirmed that the Psp Tetramer was not inherently toxic to cells. All substrates were used at $5 \mathrm{mg} / \mathrm{mL}$ unless otherwise noted. Growths are $\mathrm{n} \geq 2$, error bars show SEM (in most cases, smaller than the marker). e, Traces of RNA-seq expression data from

1299 triplicates of $B$. intestinalis grown on either glucose (Glc) or XG oligosaccharides (XGOs), illustrating overexpression of the XGO PUL.

\section{Extended Data 10.}

1303 a, Metagenomic sequencing of additional 16 cultures (S, human fecal sample) that actively grew 1304 on and degraded xanthan gum revealed two architectures of the R. UCG13. The more prevalent 1305 locus contained a GH125 insertion. The 10 additional samples with this locus architecture 1306 include: S22, S25, S39, S43, S44, S45, S49, S53, S58, and S59. b, The $B$. intestinalis xanthan 1307 locus was present in 3 additional cultures. c, Additional members of the Bacteroideceae family 1308 harbor a PUL with a GH88, GH92 and GH3 that could potentially enable utilization of XG1309 oligosaccharides.

1310 d, The GH125-containing version of the R. UCG13 xanthan locus was detected in two mouse 1311 fecal samples (M, mouse fecal sample). e, Comparison of the human and mouse RuGH5a aa 1312 sequence, showing the annotated signal peptide (SP), GH5 domain, three carbohydrate binding 1313 modules (CBMs), and multiple Listeria-Bacteroides repeat domains. f, Genetic organization and 1314 aa identity (\%) between the $B$. intestinalis xanthan locus in the original human sample and a PUL 1315 detected in a fracking water microbial community (FWMC) using LAST-searches. g, SDS1316 PAGE gel of purified enzymes with $4.5 \mu \mathrm{g}$ loaded, including ladder and the different mouse $1317 R u$ GH5a constructs. A, B, and C are all versions of the GH5 domain alone, D is a construct 1318 designed to terminate at a site homologous to the last CBM in the human $R u \mathrm{GH} 5 \mathrm{a}$, and $\mathrm{E}$ is a 1319 full-length construct of the mouse $R u$ GH5a. h, TLC of each mouse $R u$ GH5a construct incubated 1320 with XG and also odd (1, 3, 5, and 7 residues) and even (2, 4, and 6 residues) malto-

1321 oligosaccharide standards. The GH5-only constructs did not degrade XG, but constructs D and E 1322 (with regions homologous to the human $R u \mathrm{GH} 5$ a CBMs) were able to hydrolyze XG. 
bioRxiv preprint doi: https://doi.org/10.1101/2021.06.02.446819; this version posted June 2, 2021. The copyright holder for this preprint (which Figureas not certified by peer review) is the author/funder, who has granted bioRxiv a license to display the preprint in perpetuity. It is made

A
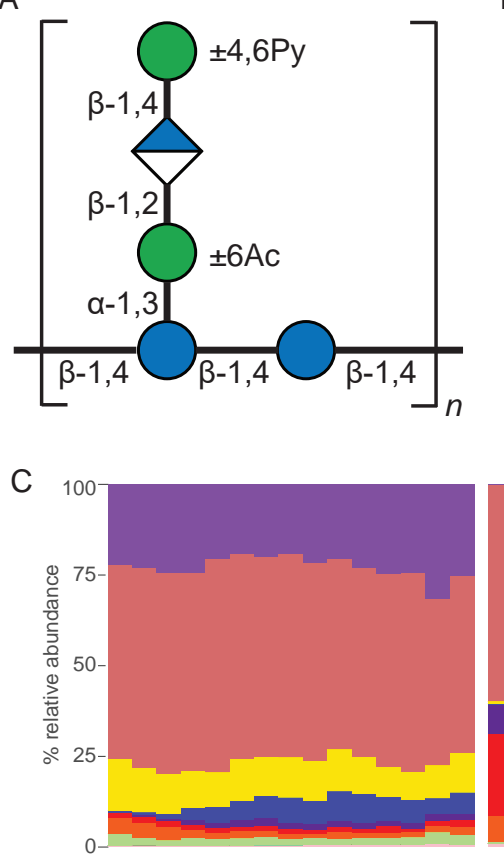

passage: $56781011121314151617181920 \mathrm{BH}$ activity: +++++++++++++++
B
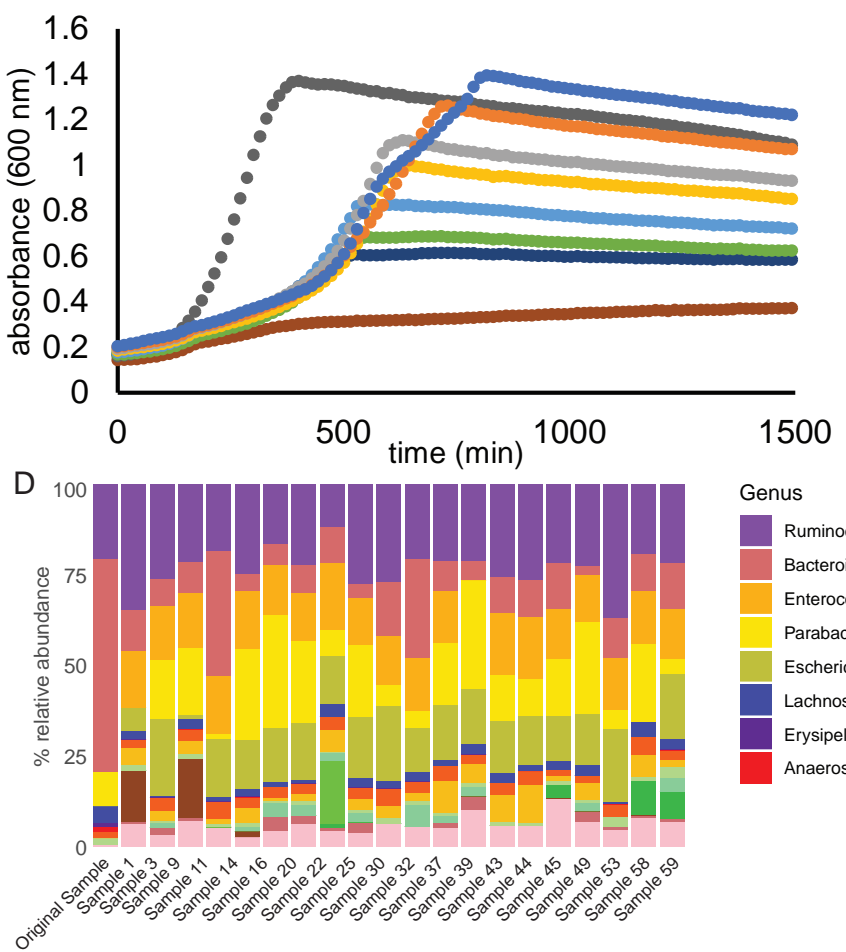

- Water

- $5 \mathrm{mg} / \mathrm{mL}$ Monosaccharides

$0.6 \mathrm{mg} / \mathrm{mL}$

- $0.9 \mathrm{mg} / \mathrm{mL}$

- $1.3 \mathrm{mg} / \mathrm{mL}$

- $1.9 \mathrm{mg} / \mathrm{mL}$

- $2.5 \mathrm{mg} / \mathrm{mL}$

- $3.8 \mathrm{mg} / \mathrm{mL}$

- $5 \mathrm{mg} / \mathrm{mL}$

Genus

Ruminococcaceae_UCG.013

Bacteroides

Enterococcus

Parabacteroides

Escherichia.Shigella

Lachnospiraceae unclassified

Erysipelotrichaceae_ge

Anaerostipes

Lachnoclostridium

Eisenbergiella

Flavonifractor

Phascolarctobacterium

Fusobacterium

Lachnospiraceae_ND3007_group

Enterobacteriaceae_unclassified

Erysipelatoclostridium

Other 


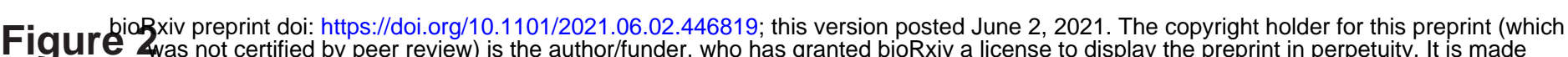
4was not certified by peer review) is the author/funder, who has granted bioRxiv a license to display the preprint in perpetuity. It is made MAG2 (R. UCG13) XG PUL available under aCC-BY 4.0 International license.

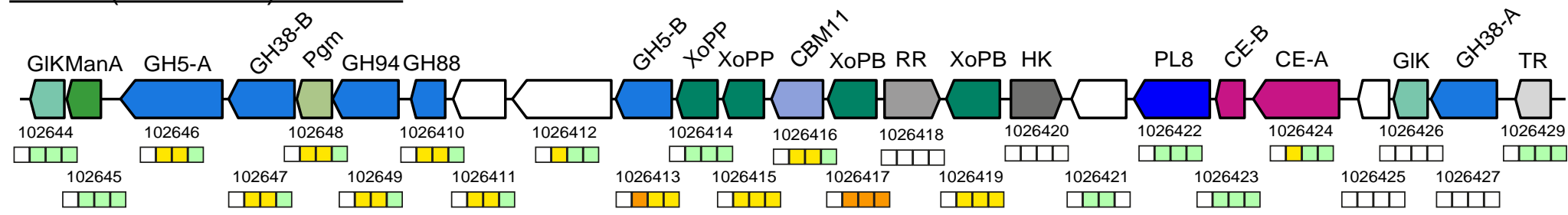

\section{MAG1 (B. intestinalis) XG PUL}

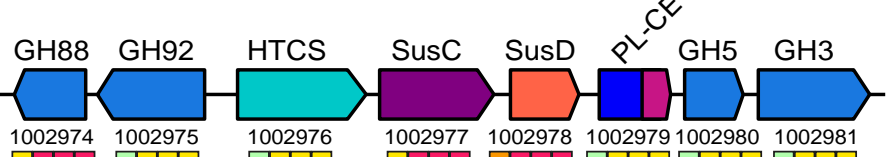

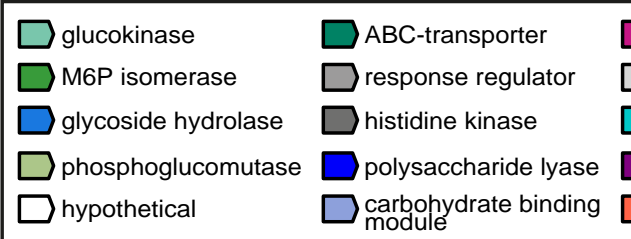

carbohydrate esterase

$\square$ transcriptional regulator

$\checkmark$ component system

SusC (transporter)

SusD (binding protein)
TPM

$\square$ above 750

$\square 251-750$

$\square$ 101-250

$\square 16-100$

$\square$ 0-15 
bioRxiv preprint doi: https://doi.org/10.1101/2021.06.02.446819; this version posted June 2, 2021. The copyright holder for this preprint (which was not certified by peer review) is the author/funder, who has granted bioRxiv a license to display the preprint in perpetuity. It is made available under aCC-BY 4.0 International license.

\section{Figure 3}
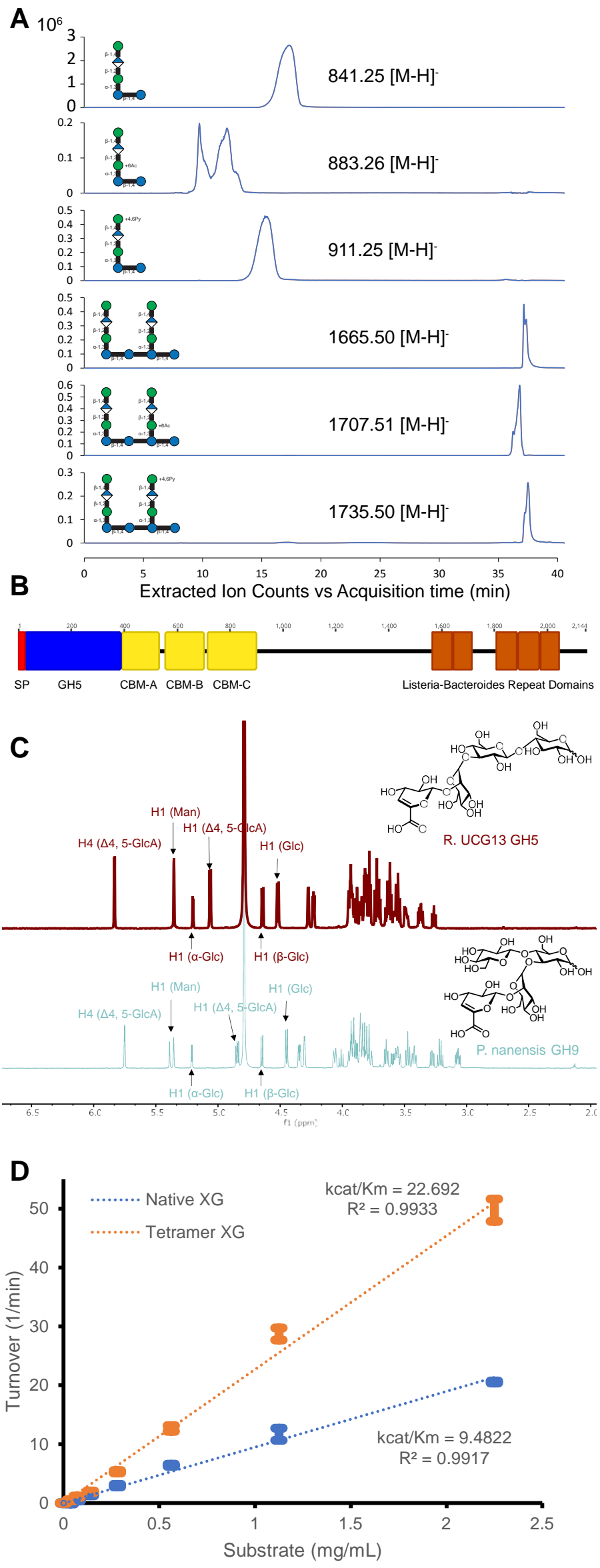
bioRxiv preprint doi: https://doi.org/10.1101/2021.06.02.446819; this version posted June 2, 2021. The copyright holder for this preprint (which was not certified by peer review) is the author/funder, who has granted bioRxiv a license to display the preprint in perpetuity. It is made

\section{Figure 4} available under aCC-BY 4.0 International license.

\section{A}
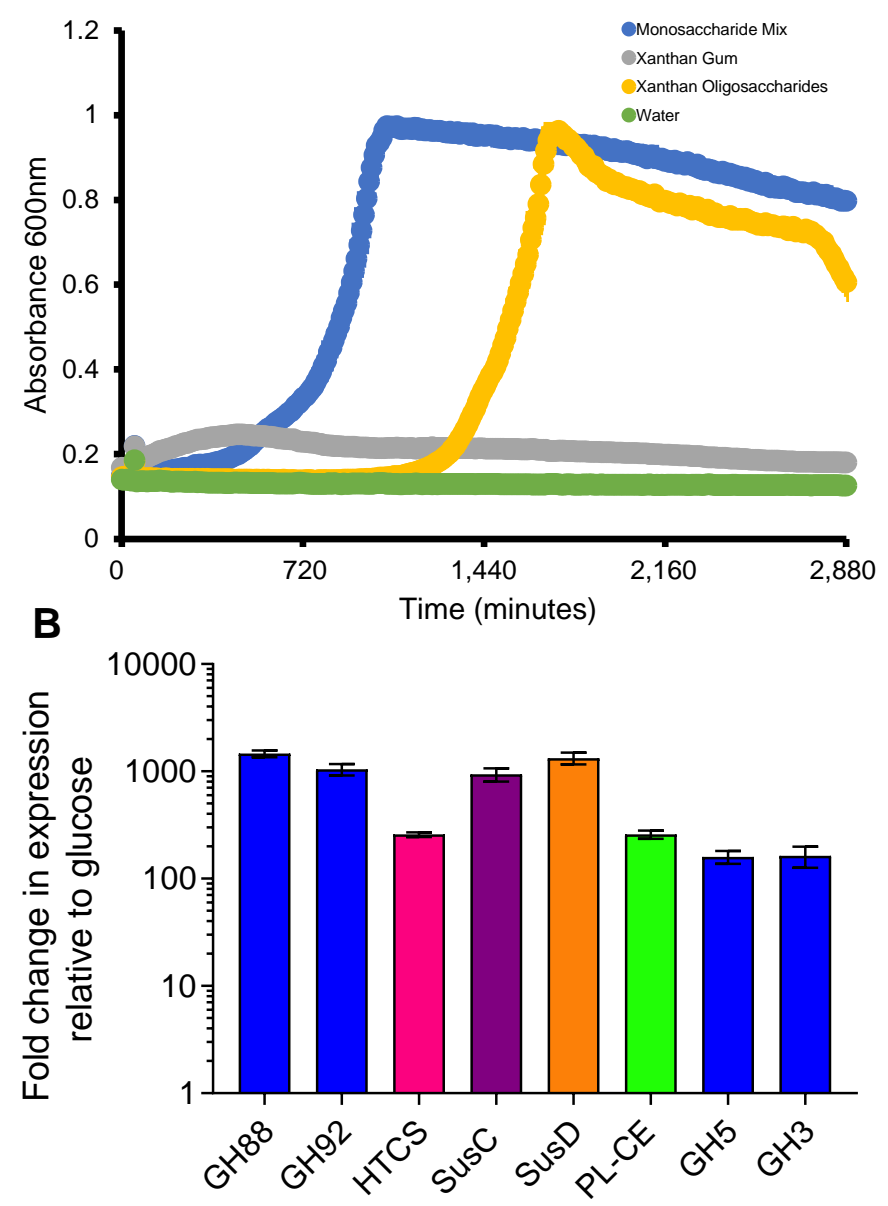

Gene in B. intestinalis PUL 
Figure 5

Chinese 1 . who has granted bioRxiv a license to display the preprint in perpetuity. It is made

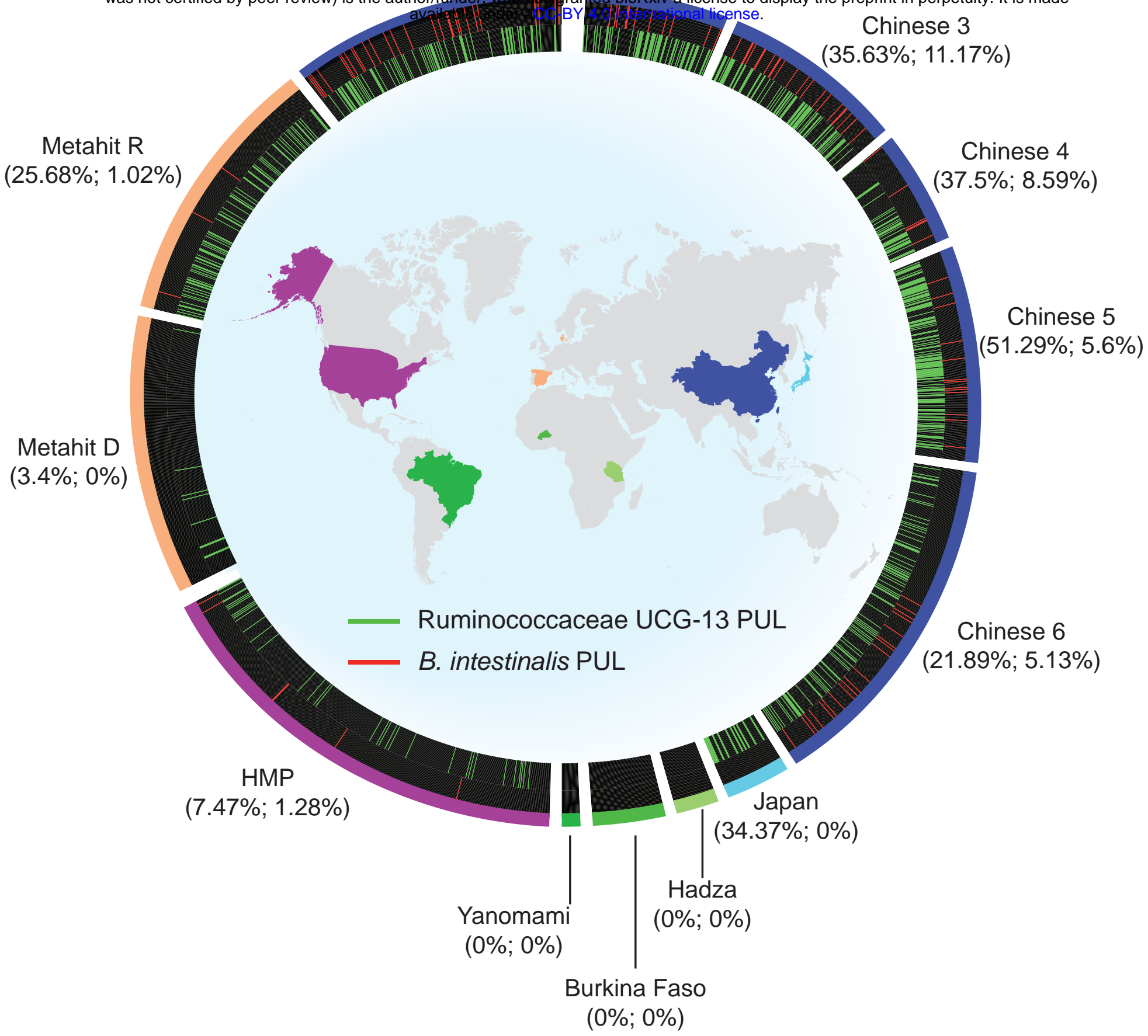




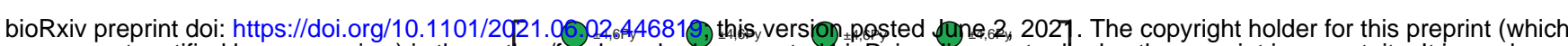

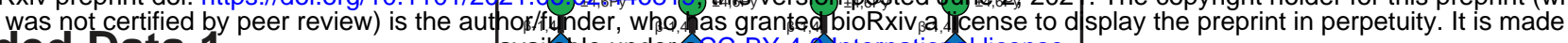
Extended Data 1
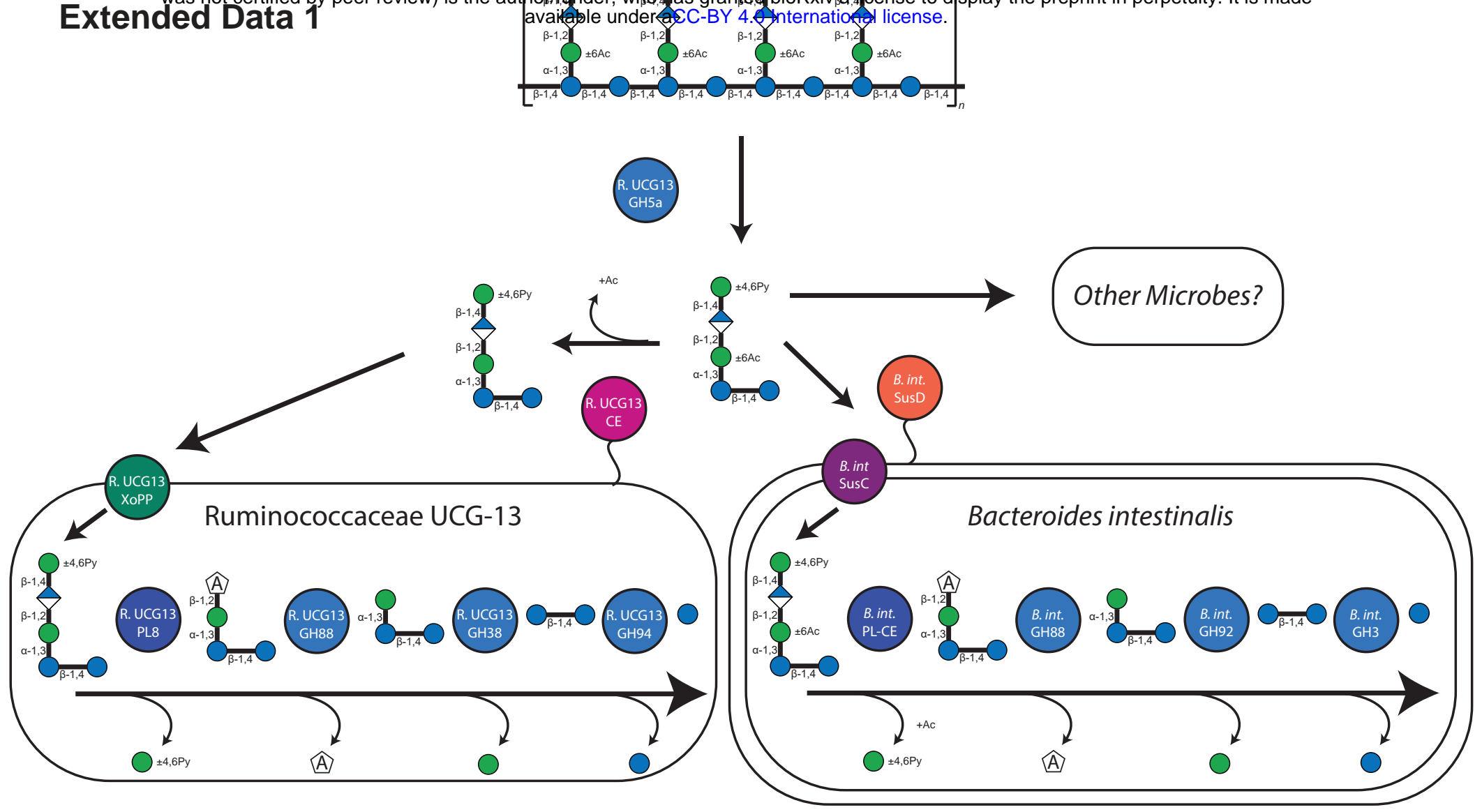
bioRxiv preprint doi: https://doi.org/10.1101/2021.06.02.446819; this version posted June 2, 2021. The copyright holder for this preprint (which was not certified by peer review) is the author/funder, who has granted bioRxiv a license to display the preprint in perpetuity. It is made

\section{Extended Data 2}

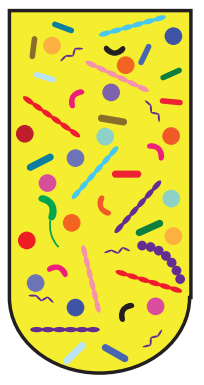

$10^{6}$

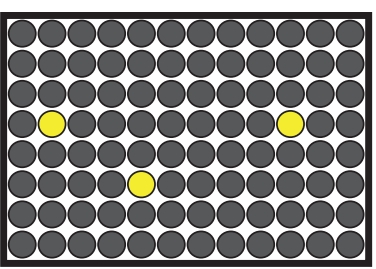

93/96

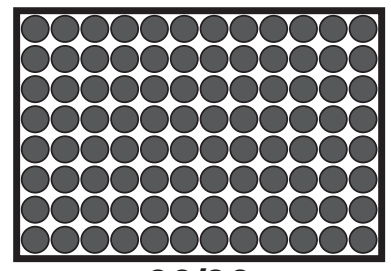

96/96
Dilution Factor

Xanthan Gum

Monosaccharides available under aCC-BY 4.0 International license.

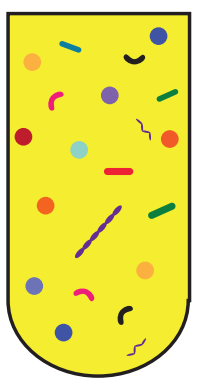

$10^{7}$

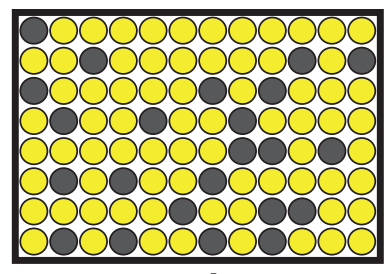

23/96

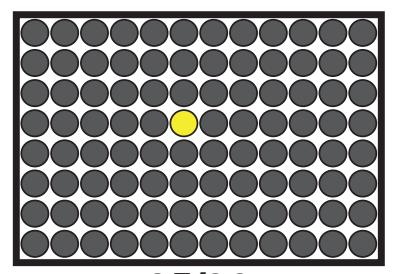

95/96

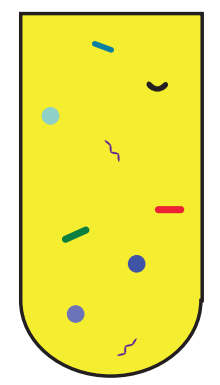

$10^{8}$

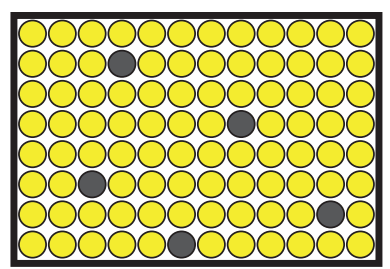

5/96

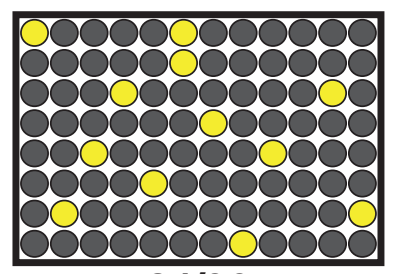

84/96

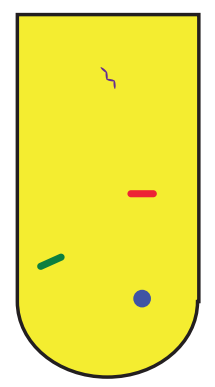

$10^{9}$

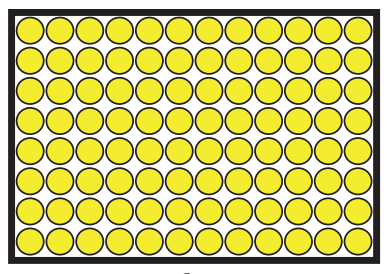

$0 / 96$

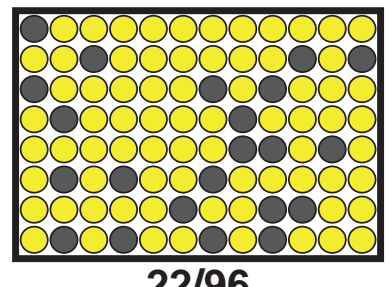

22/96

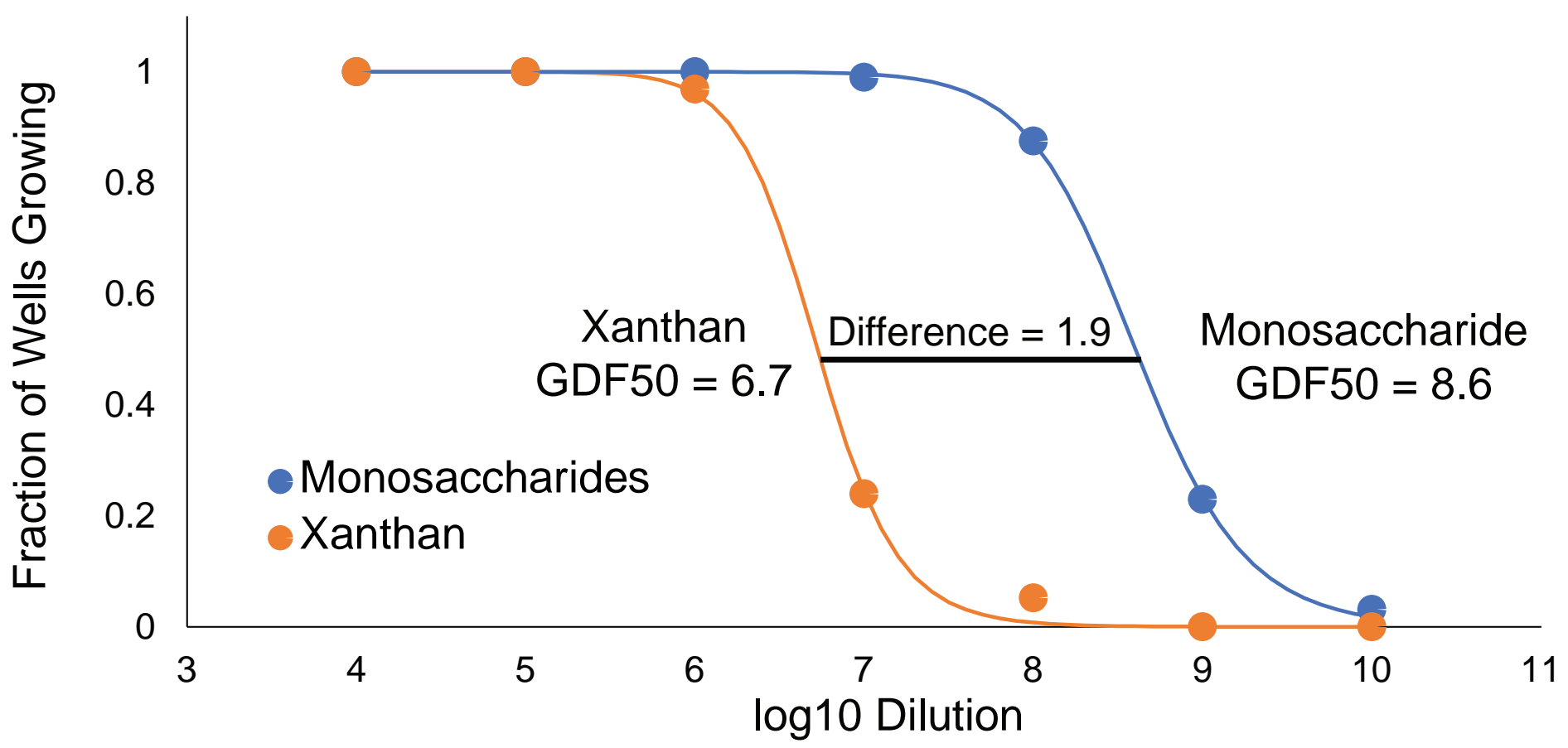


Extended Data 3

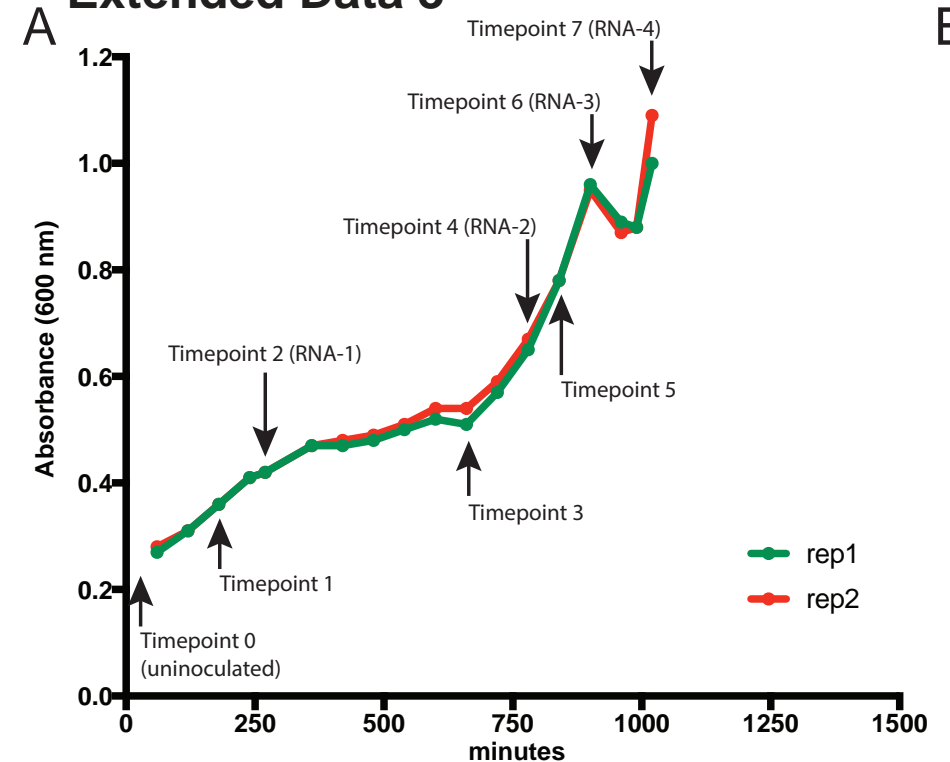

Replicate 1 composition

\begin{tabular}{lll}
\hline Samples & Glucose (\%) & Mannose (\%) \\
\hline Timepoint 0 & $54.82 \pm 0.12$ & $45.18 \pm 0.49$ \\
Timepoint 1 & $54.37 \pm 1.10$ & $45.63 \pm 1.05$ \\
Timepoint 2 & $55.52 \pm 0.78$ & $44.48 \pm 0.77$ \\
Timepoint 3 & $54.97 \pm 0.57$ & $45.03 \pm 0.57$ \\
Timepoint 4 & $56.73 \pm 0.42$ & $43.27 \pm 0.29$ \\
Timepoint 5 & $55.84 \pm 0.14$ & $44.26 \pm 0.11$ \\
\hline
\end{tabular}

Replicate 2 composition

\begin{tabular}{lll}
\hline Samples & Glucose (\%) & Mannose (\%) \\
\hline Timepoint 0 & $54.82 \pm 0.12$ & $45.18 \pm 0.49$ \\
Timepoint 1 & $54.99 \pm 0.65$ & $45.01 \pm 1.03$ \\
Timepoint 2 & $55.04 \pm 0.55$ & $44.96 \pm 0.84$ \\
Timepoint 3 & $55.24 \pm 0.32$ & $44.76 \pm 0.54$ \\
Timepoint 4 & $55.99 \pm 0.17$ & $44.01 \pm 1.32$ \\
Timepoint 5 & $55.28 \pm 0.88$ & $44.72 \pm 1.09$ \\
\hline
\end{tabular}

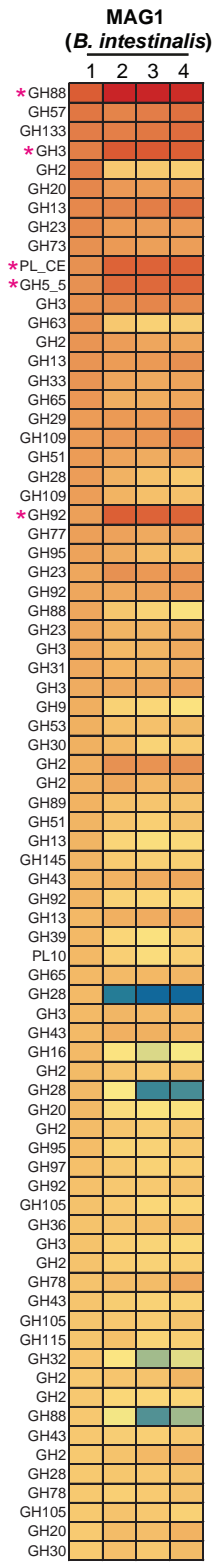

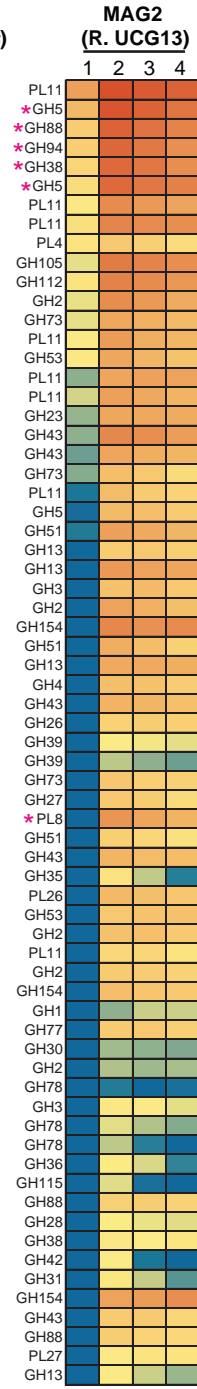

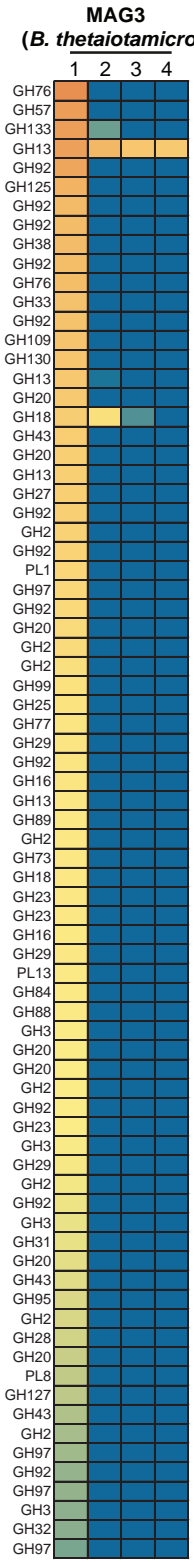

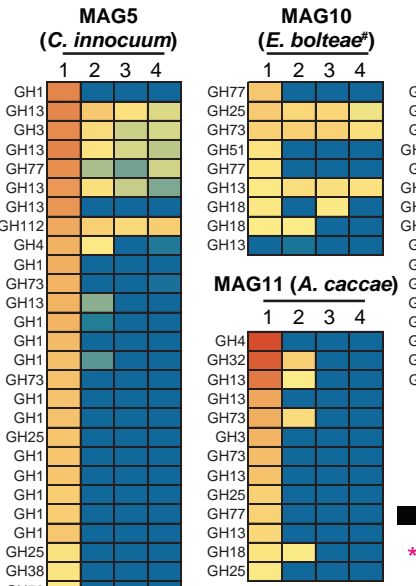

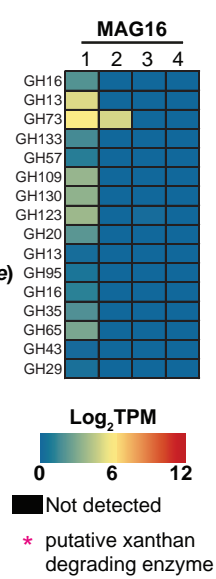

MAG12 (B. uniformis)

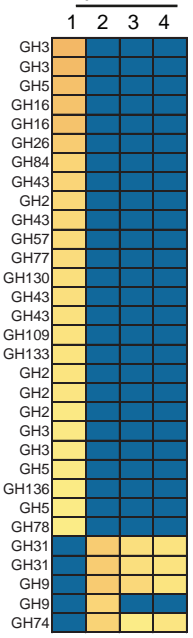

MAG13 (E. lenta)

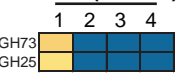

MAG9 (F. plautii)

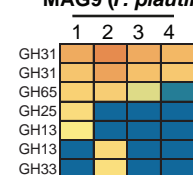

MAG14 (E. piscisicarius ${ }^{\#)}$ 
bioRxiv preprint doi: https://doi.org/10.1101/2021.06.02.446819; this version posted June 2, 2021. The copyright holder for this preprint (which was not certified by peer review) is the author/funder, who has granted bioRxiv a license to display the preprint in perpetuity. It is made

\section{Extended Data 4} available under aCC-BY 4.0 International license.

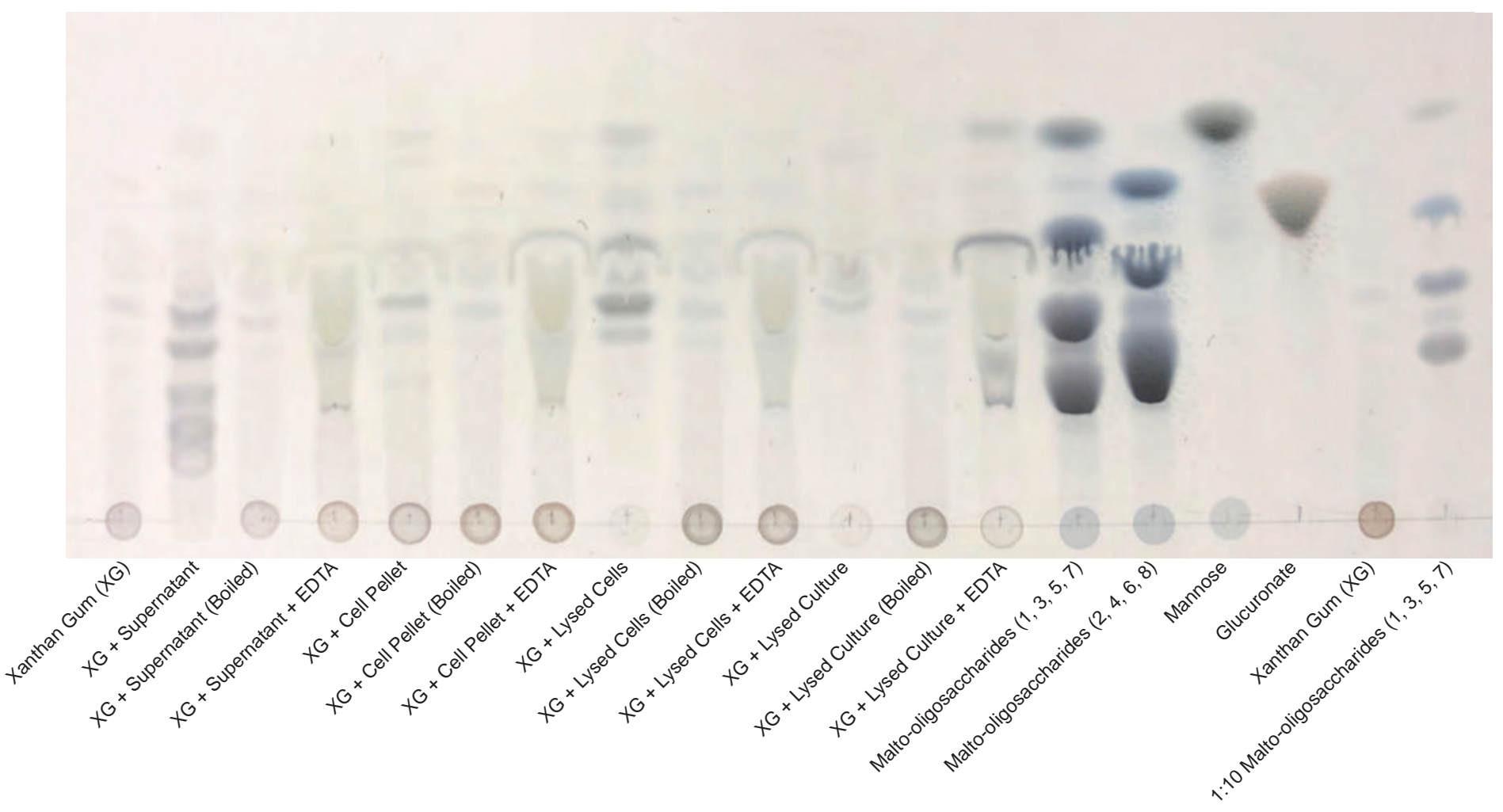


bioxiv preprint doi https://dgiorg/10.1101/2021.06.02.446819; this version posted June 2, 2021. The copyright holder for this preprint (which

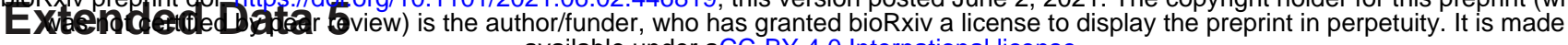
available under aCC-BY 4.0 International license.

A

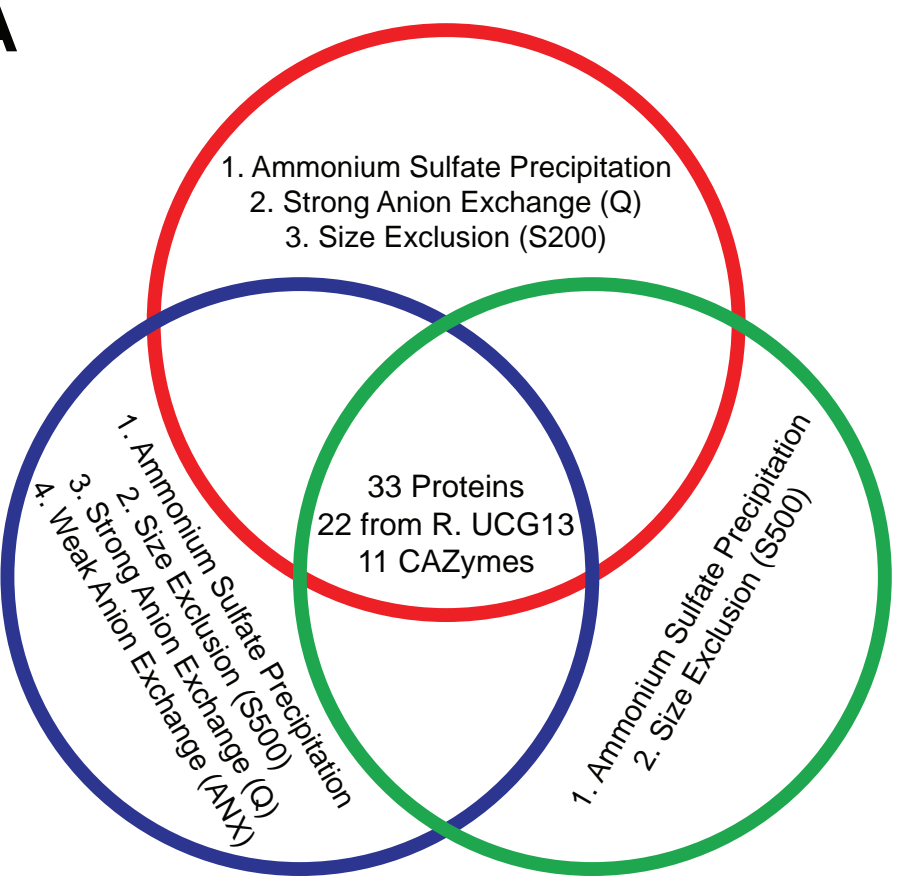

B

C

\begin{tabular}{|c|c|c|c|c|c|c|}
\hline Protein ID & Protein Annotation & Species Prediction & CAZyme Annotation & \#Peptides (E95) & \#Peptides (E97) & \#Peptides (E131) \\
\hline Ga0308426_1011562 & beta-galactosidase/beta-glucuronidase & Ruminococcaceae UCG-13 & $\mathrm{GH} 2$ & 102 & 110 & 114 \\
\hline Ga0308426_10042164 & alpha-tubulin suppressor-like RCC1 family protein & Ruminococcaceae UCG-13 & \#N/A & 106 & 196 & 8 \\
\hline Ga0308426_102646 & cellulase (glycosyl hydrolase family 5)/List-Bact-rpt repeat protein & Ruminococcaceae UCG-13 & GH5 & 78 & 94 & 91 \\
\hline Ga0308426_100982 & uncharacterized protein RhaS with RHS repeats & Ruminococcaceae UCG-13 & \#N/A & 120 & 94 & 13 \\
\hline Ga0308426_1061813 & uncharacterized protein YjdB & Ruminococcaceae UCG-13 & PL11 & 71 & 64 & 61 \\
\hline Ga0308426_1039440 & beta-xylosidase & Ruminococcaceae UCG-13 & GH43_4-GH43_29 & 75 & 61 & 49 \\
\hline Ga0308426_110622 & $\mathrm{N}$-acetylneuraminic acid mutarotase & Clostridium formicaceticum A1, DSM92 & \#N/A & 66 & 65 & 49 \\
\hline Ga0308426_1046623 & O-glycosyl hydrolase & Ruminococcaceae UCG-13 & GH95-GH43_22-GH30_5 & 70 & 53 & 12 \\
\hline Ga0308426_1044429 & S-layer family protein & Ruminococcaceae UCG-13 & \#N/A & 55 & 26 & 44 \\
\hline Ga0308426_1010896 & SdrD B-like protein & Anaerostipes hadrus & \#N/A & 75 & 22 & 26 \\
\hline Ga0308426_115143 & List-Bact-rpt repeat protein & Lachnospiraceae bacterium sp. 7_1_58FAA & \#N/A & 34 & 33 & 31 \\
\hline Ga0308426_110623 & S-layer family protein & Ruminiclostridium thermosuccinogenes DSM 5809 & \#N/A & 46 & 44 & 5 \\
\hline Ga0308426_108116 & S-layer family protein & Ruminococcaceae UCG-13 & \#N/A & 55 & 33 & 6 \\
\hline Ga0308426_1009815 & formate C-acetyltransferase & Ruminococcaceae UCG-13 & \#N/A & 48 & 16 & 16 \\
\hline Ga0308426_10026226 & S-layer family protein & Ruminococcaceae UCG-13 & \#N/A & 30 & 10 & 17 \\
\hline Ga0308426_1046628 & alpha-L-arabinofuranosidase & Ruminococcaceae UCG-13 & GH51-GH43_34 & 24 & 14 & 19 \\
\hline Ga0308426_1026413 & cellulase (glycosyl hydrolase family 5) & Ruminococcaceae UCG-13 & GH5 & 27 & 18 & 9 \\
\hline Ga0308426_109238 & hypothetical protein & Bacteroides intestinalis & \#N/A & 26 & 10 & 18 \\
\hline Ga0308426_1011531 & Ig-like protein group 3 & Ruminococcaceae UCG-13 & PL11 & 27 & 9 & 15 \\
\hline Ga0308426_1015472 & chaperonin GroEL & Clostridium saccharolyticum & \#N/A & 26 & 12 & 7 \\
\hline Ga0308426_10042105 & hypothetical protein & Ruminococcaceae UCG-13 & СBM66 & 25 & 14 & 5 \\
\hline Ga0308426_1038838 & surface antigen & Ruminococcaceae UCG-13 & COH1-DOC-COH1 & 24 & 12 & 7 \\
\hline Ga0308426_1000896 & putative aldouronate transport system substrate-binding protein & Ruminococcaceae UCG-13 & \#N/A & 28 & 5 & 3 \\
\hline Ga0308426_10026230 & carboxyl-terminal processing protease & Ruminococcaceae UCG-13 & \#N/A & 20 & 1 & 14 \\
\hline Ga0308426_10042104 & $\begin{array}{l}\text { copper amine oxidase-like protein/concanavalin A-like lectin/glucanase } \\
\text { superfamily protein }\end{array}$ & Ruminococcaceae UCG-13 & \#N/A & 19 & 1 & 15 \\
\hline Ga0308426_109371 & hypothetical protein & Clostridium sp. ATCC BAA-442 & \#N/A & 22 & 7 & 5 \\
\hline Ga0308426_10026260 & hypothetical protein & Ruminococcaceae UCG-13 & \#N/A & 22 & 7 & 2 \\
\hline Ga0308426_1000893 & rhamnogalacturonyl hydrolase YesR & Ruminococcaceae UCG-13 & GH105 & 16 & 8 & 1 \\
\hline Ga0308426_112844 & hypothetical protein & Bacteroides pectinophilus ATCC 43243 & \#N/A & 18 & 1 & 6 \\
\hline Ga0308426_108113 & copper amine oxidase-like protein/leucine rich repeat (LRR) protein & Ruminococcaceae UCG-13 & \#N/A & 15 & 2 & 3 \\
\hline Ga0308426_1013474 & hypothetical protein & Bacteroides intestinalis & \#N/A & 10 & 3 & 1 \\
\hline Ga0308426_10029134 & hypothetical protein & Bacteroides intestinalis & \#N/A & 7 & 1 & 2 \\
\hline Ga0308426_115141 & S-layer family protein & Flavonifractor plautii YL31 & \#N/A & 6 & 1 & 2 \\
\hline
\end{tabular}


bioRxiv preprint doi: https://doi.org/10.1101/2021.06.02.446819; this version posted June 2, 2021. The copyright holder for this preprint (which was not certified by peer review) is the author/funder, who has granted bioRxiv a license to display the preprint in perpetuity. It is made

\section{Extended Data 6}

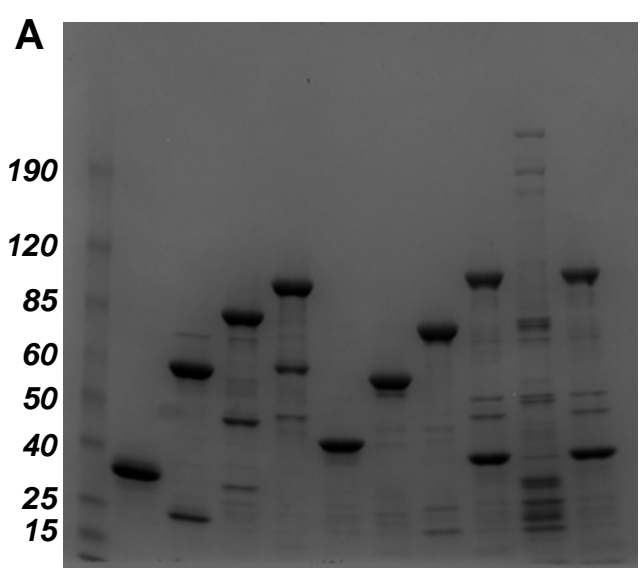

$\begin{array}{llllllllll}1 & 2 & 3 & 4 & 5 & 6 & 7 & 8 & 9 & 10\end{array}$

C

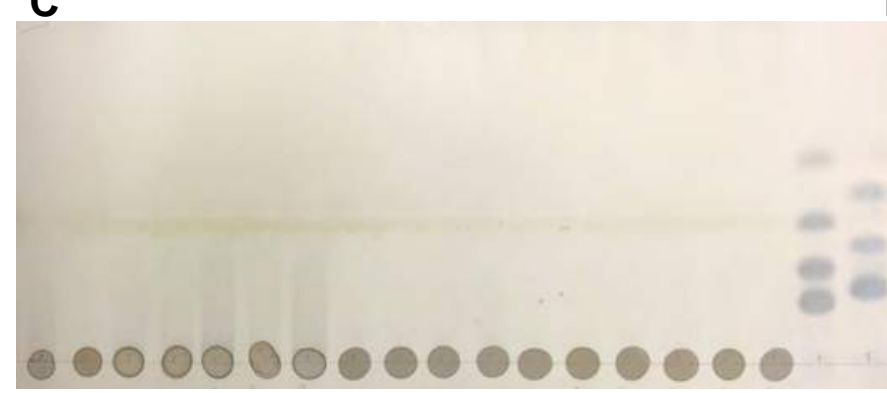

1. RuGH5b (GH5 only) 4. RuGH5b (full) 5. RuGH5a (GH5 only) 9. RuGH5a (full)
B 2. RuGH5b (GH5+CBM-A) 3. RUGH5b (GH5+CBM-A/B) 6. RUGH5a (GH5+CBM-A) 7. RuGH5a (GH5+CBM-A/B) 8. RuGH5a (GH5+CBMs) 10. RuGH5a (GH5+CBMs)

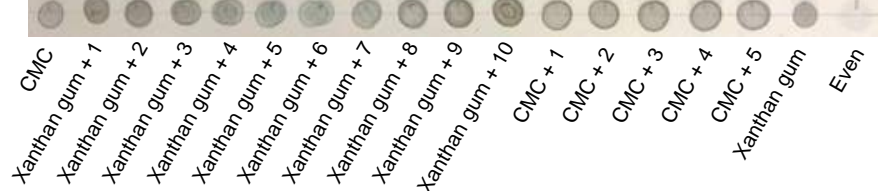

D
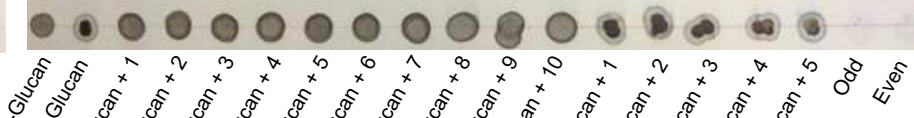

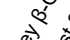

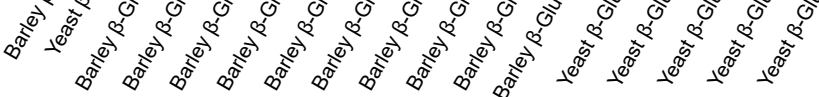

E

F

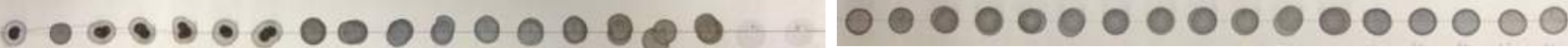

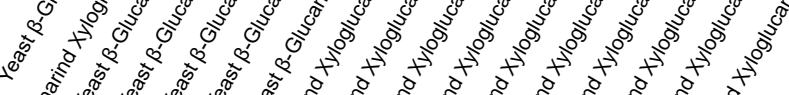

G

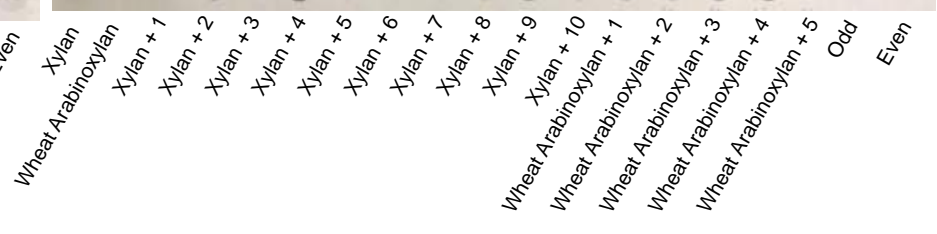


bioRxiv preprint doi: https://doi.org/10.1101/2021.06.02.446819; this version posted June 2, 2021. The copyright holder for this preprint (which was not certified by peer review) is the author/funder, who has granted bioRxiv a license to display the preprint in perpetuity. It is made

\section{Extended Data 7} available under aCC-BY 4.0 International license.

A

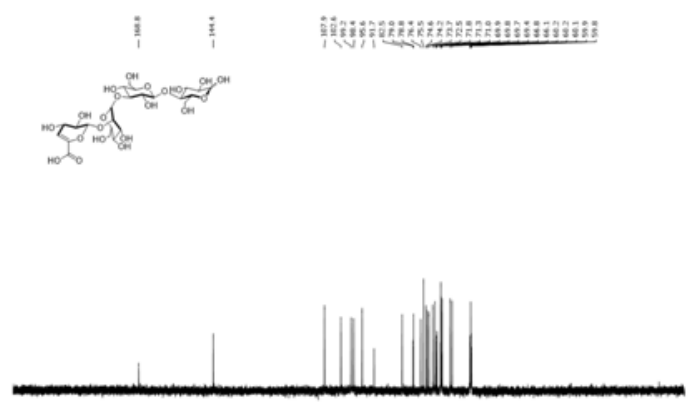

C
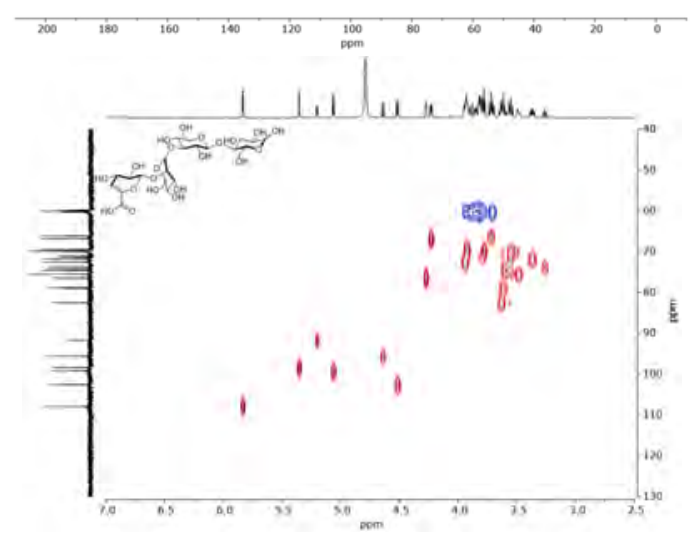

$\mathbf{E}$

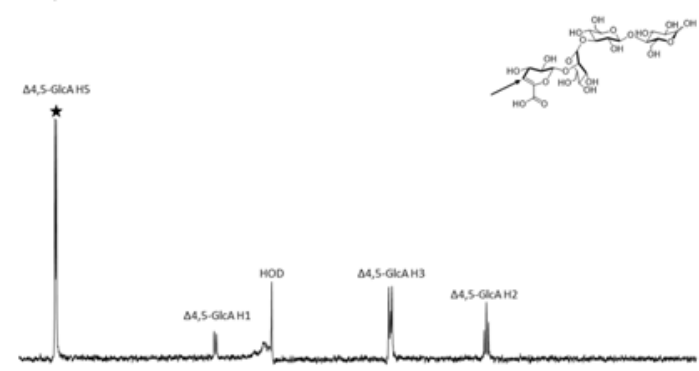

G
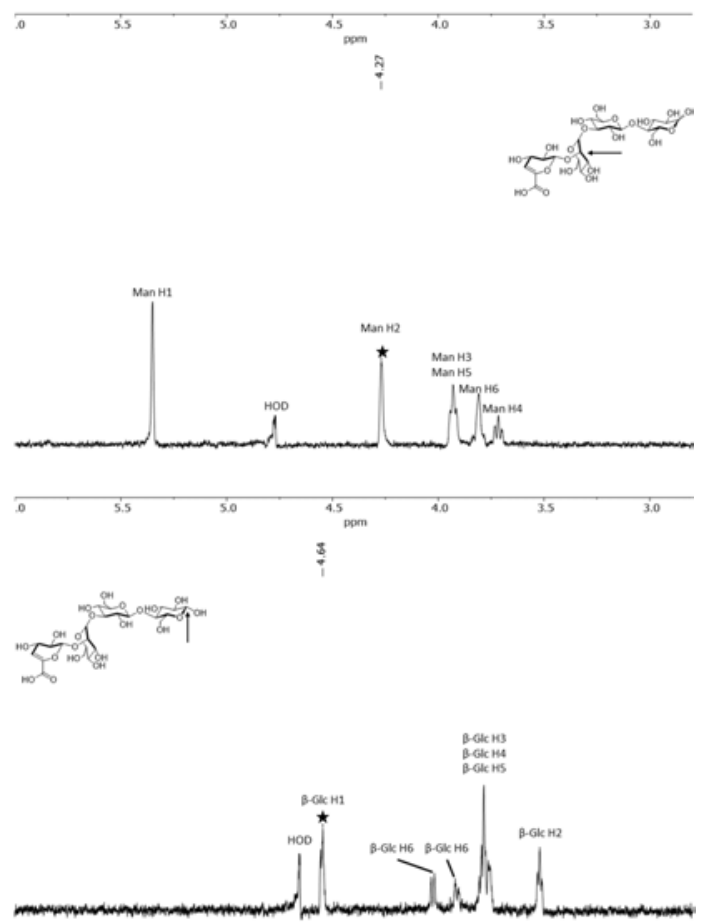

B

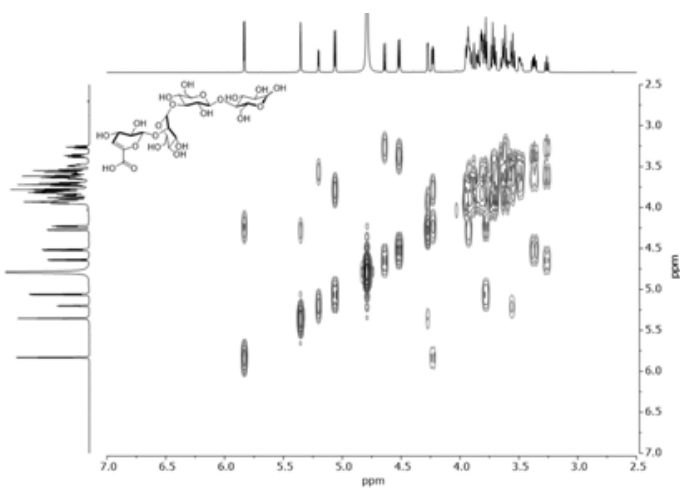

D

$\mathbf{F}$

H
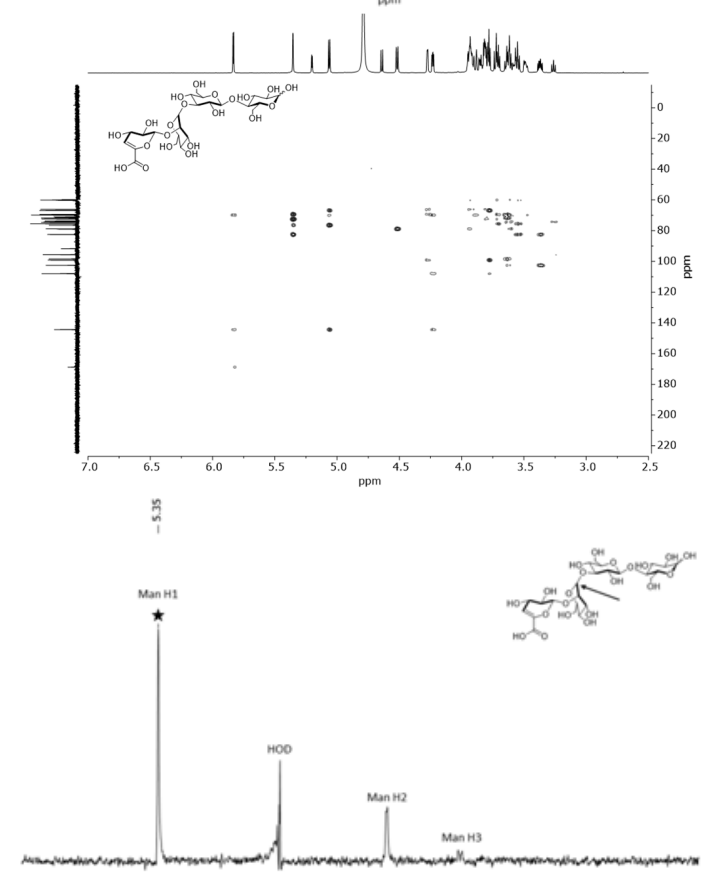

J
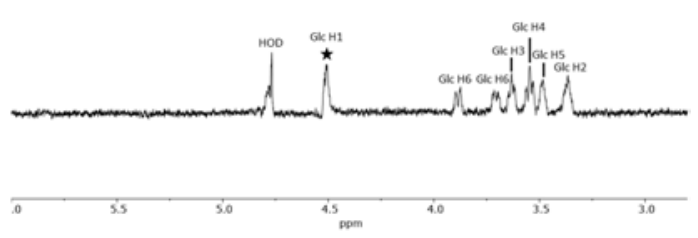

쟁

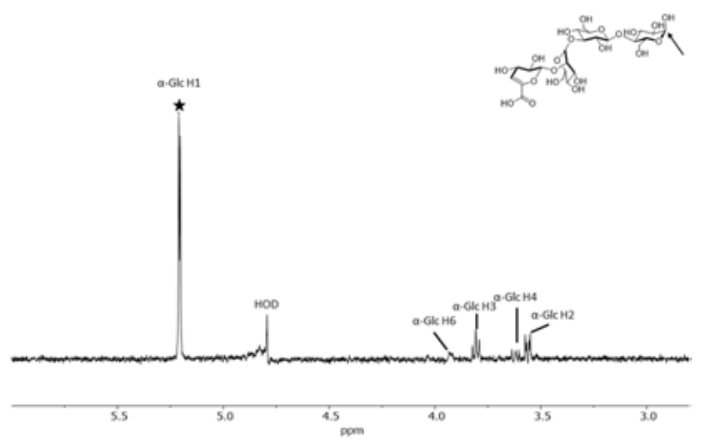




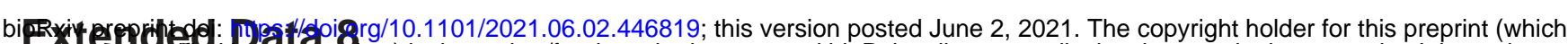
was Mot cerffied by peer review) is the author/funder, who has granted bioRxiv a license to display the preprint in perpetuity. It is made
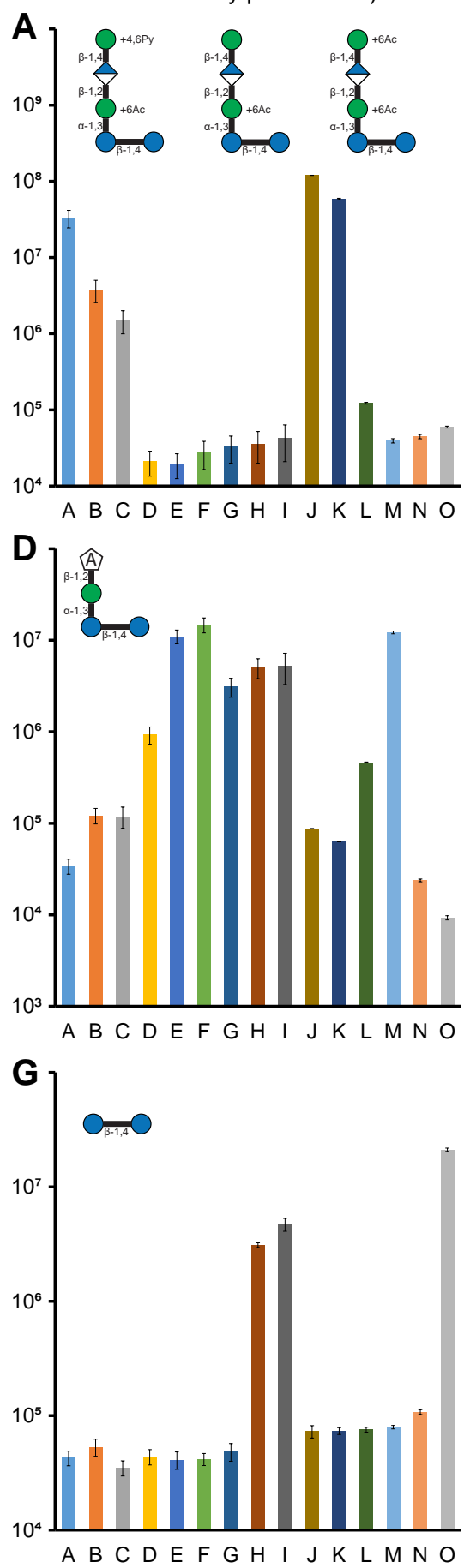

J

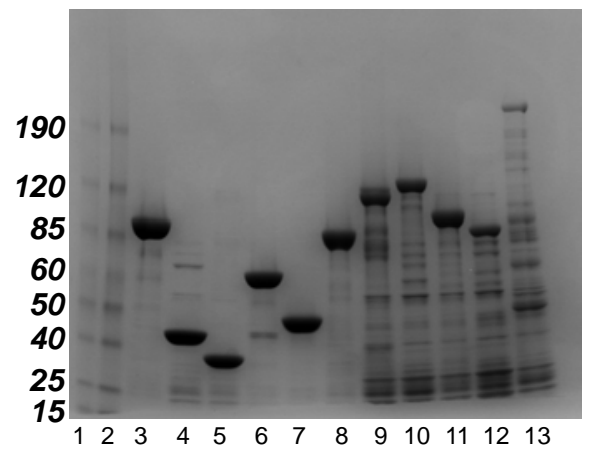

avaigble under aCC-BY 4.0 International license.

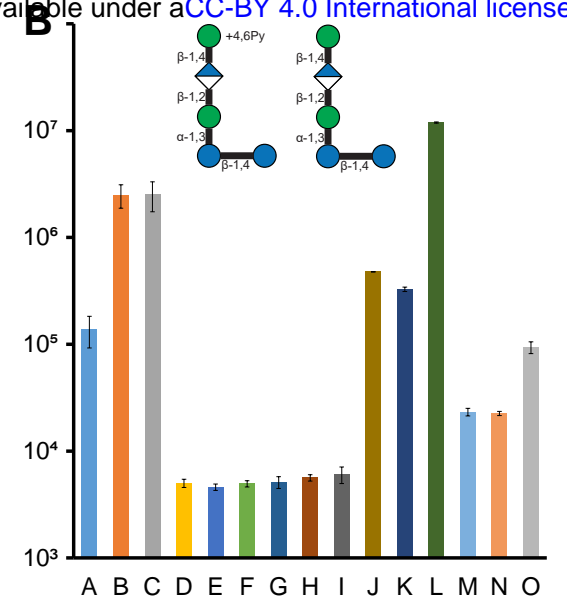

E
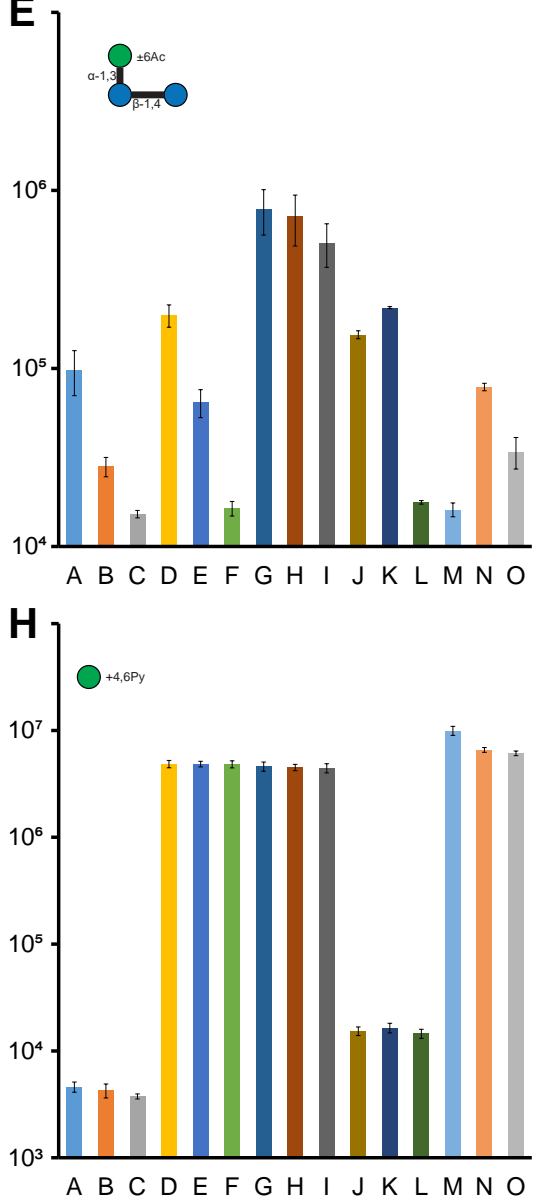

K

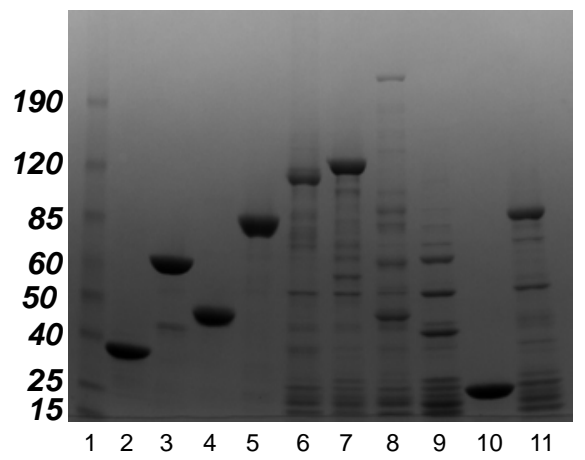

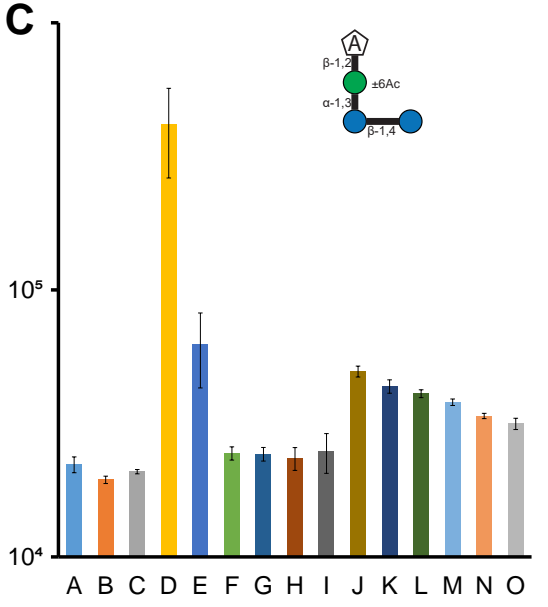

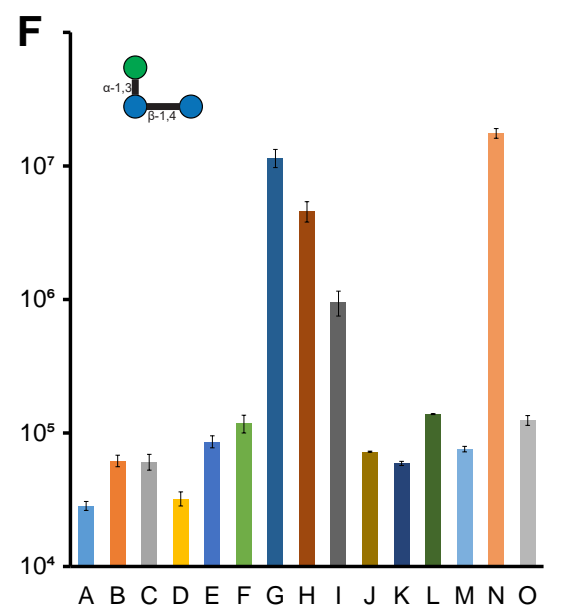

I

Ruminococcaceae UCG13 Reactions

A, No enzymes

B, CE-A

C, CE-B

$D, P L 8$

E, PL8 + CE-A

$F, P L 8+C E-B$

G, $\mathrm{PL} 8+\mathrm{CE}-\mathrm{A}+\mathrm{CE}-\mathrm{B}+\mathrm{GH} 88$

$\mathrm{H}, \mathrm{PL} 8+\mathrm{CE}-\mathrm{A}+\mathrm{CE}-\mathrm{B}+\mathrm{GH} 88+\mathrm{GH} 38-\mathrm{A}$ I, PL8 + CE-A + CE-B + GH88 + GH38-B

Bacteroides intestinalis Reactions

J, No enzymes

$\mathrm{K}, \mathrm{PL}$-only

L, PL-CE

$M, P L-C E+$ Bacillus PL8

N, PL-CE + GH88 + Bacillus PL8

$\mathrm{O}, \mathrm{PL}-\mathrm{CE}+\mathrm{GH} 88+\mathrm{GH} 92+$ Bacillus PL8

$\mathbf{L}$

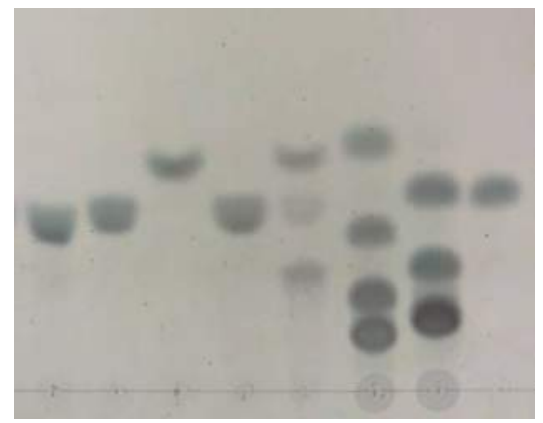

A B C D E F G H 
bioRxiv preprint doi: https://doi.org/10.1101/2021.06.02.446819; this version posted June 2, 2021. The copyright holder for this preprint (which

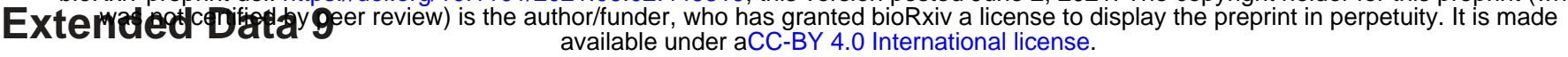

\section{A}
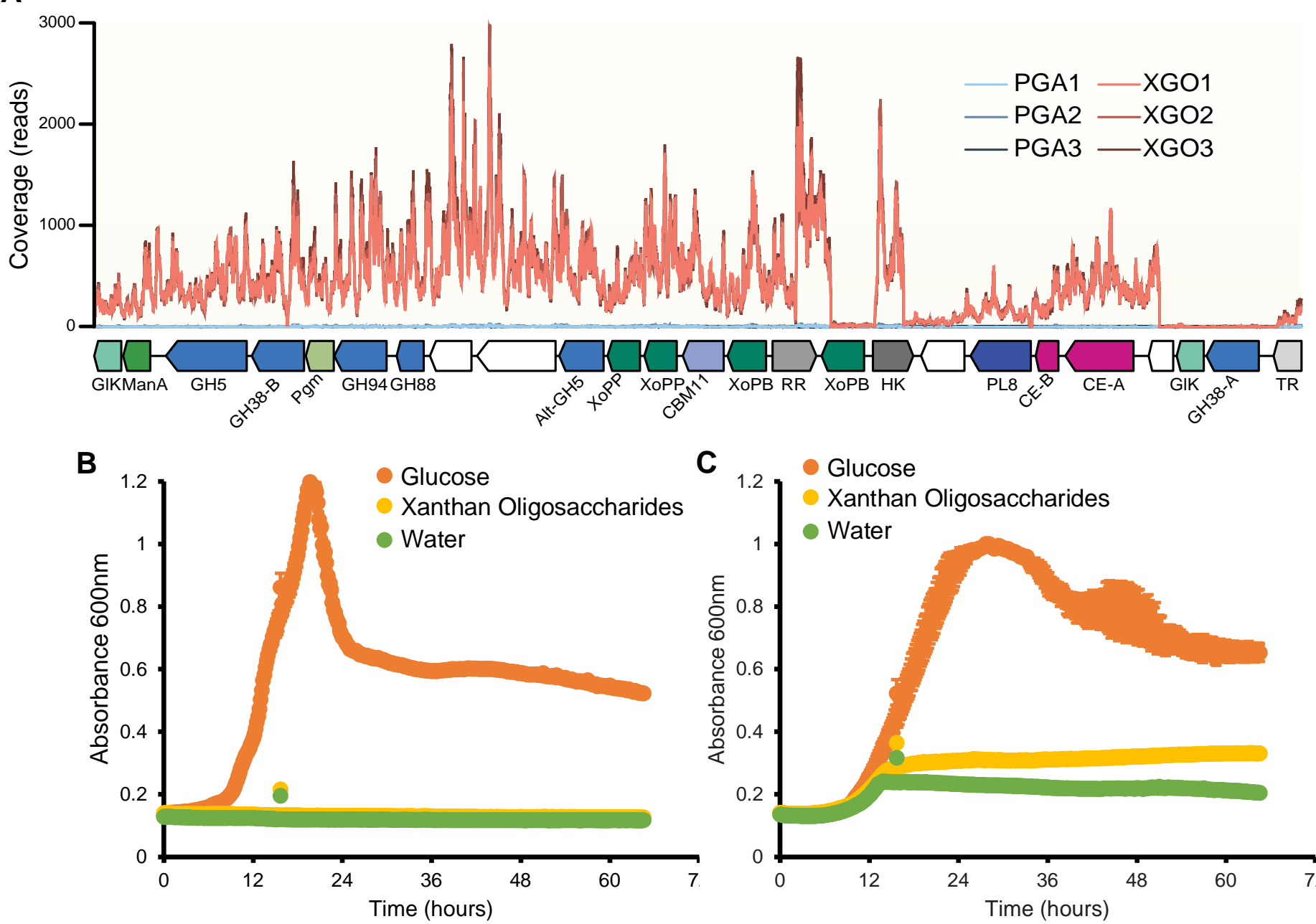

C $1.2 \begin{aligned} & \text { Glucose } \\ & \text { Xanthan Oligosaccharides } \\ & \text { Water }\end{aligned}$
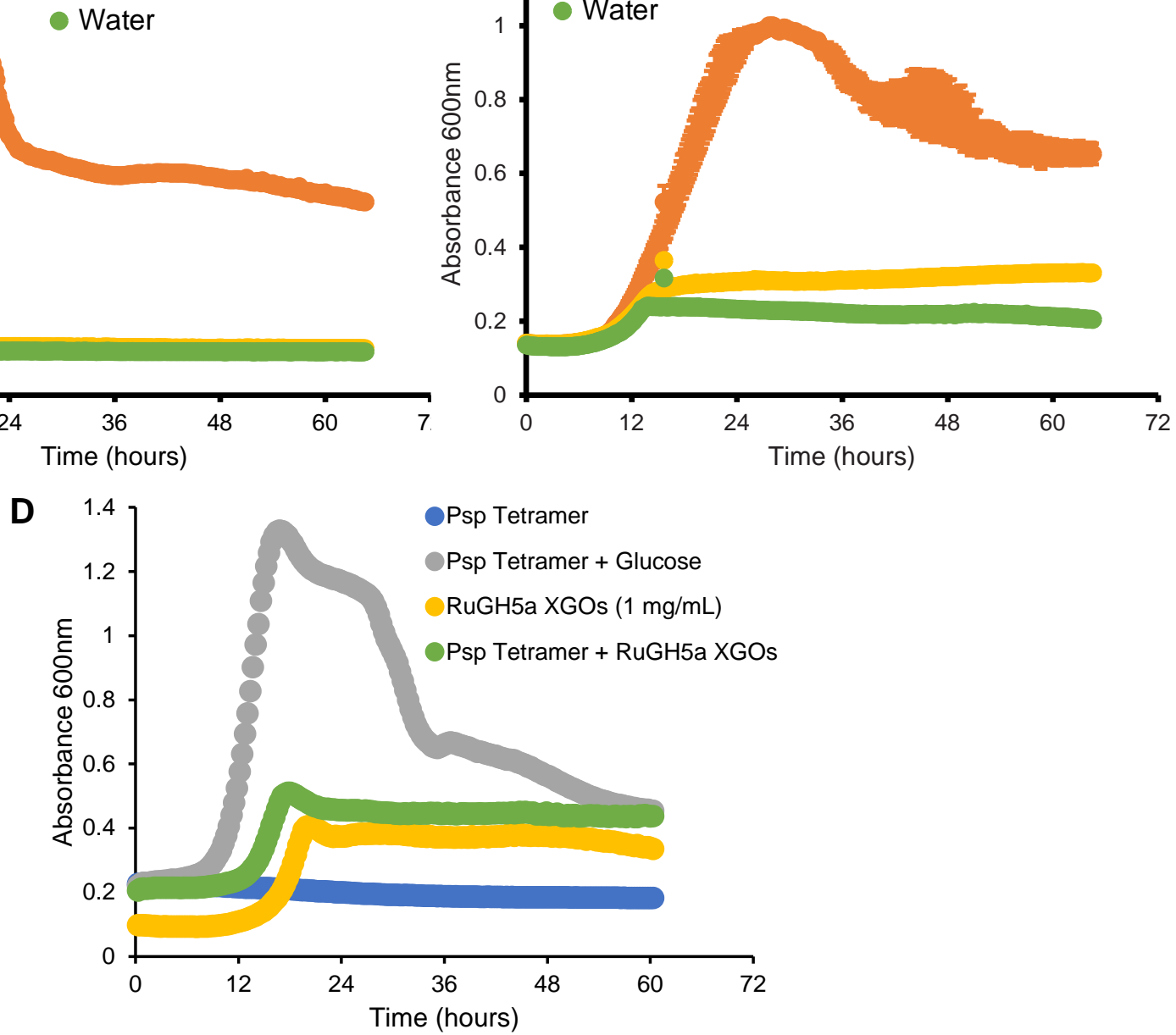

E

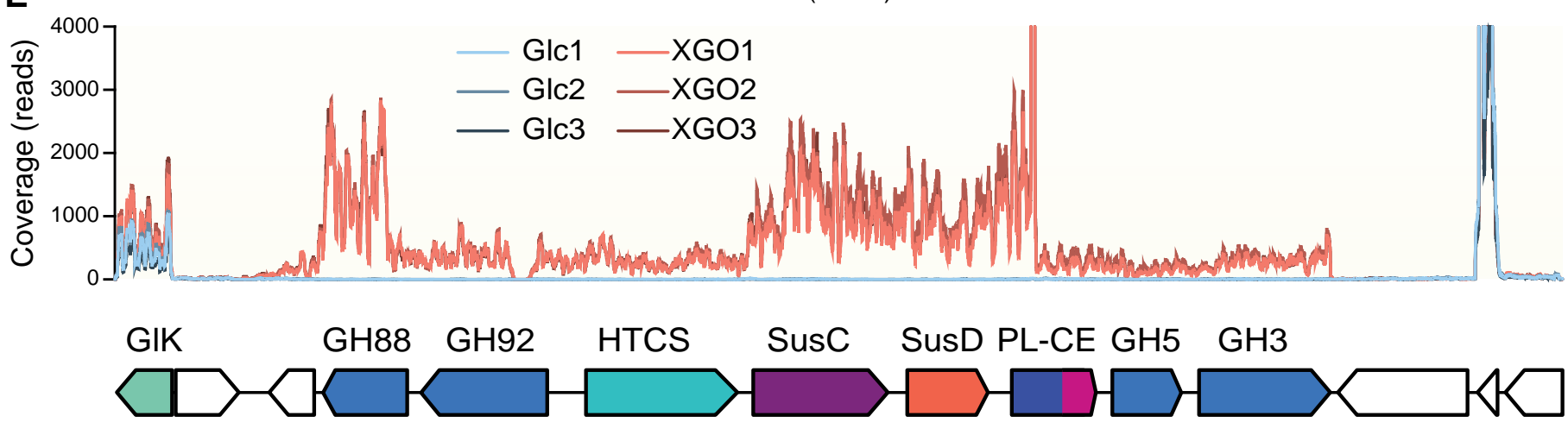


a MAG2 (R. UCG13) XG PUL

\section{Extended Data 10}

GIK ManA GH5 GH38-B Pgm GH94 GH88 GH5 XoPP XoPP CBM11 XoBP RR XOBP HK PL8 CE-B CE-A GIK GH38-A TR

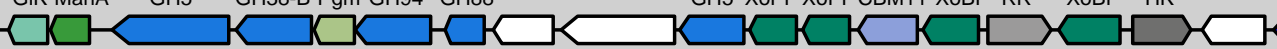
PL8

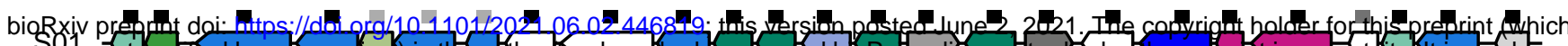

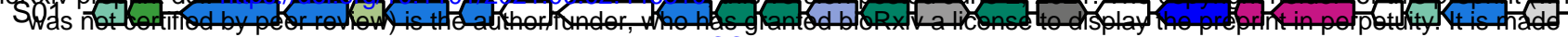

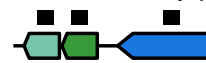

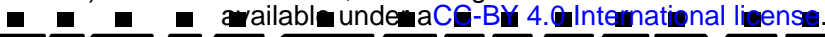

$\square$

s03 $\square \mathrm{a}<\mathrm{ar}$

S09*

品

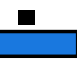

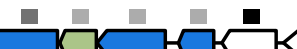

S11

S16*

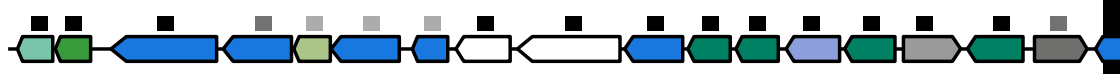

$\longrightarrow \square \square+\square \square \square$

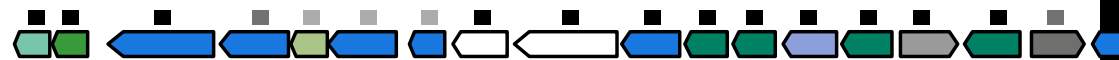

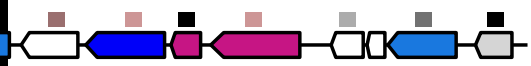
samples

b

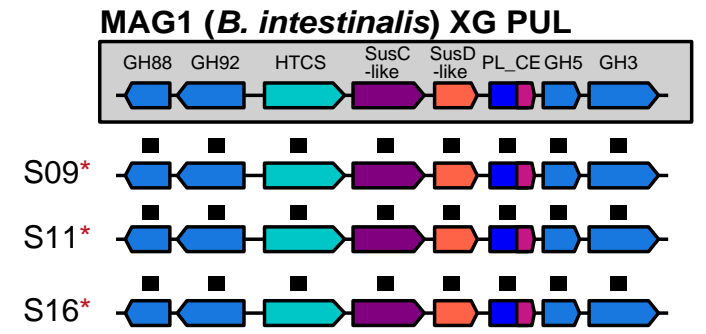

C S59

$\mathrm{S} 1^{\#}$ HTCS

d MAG2 (R. UCG13, human sample) XG PUL

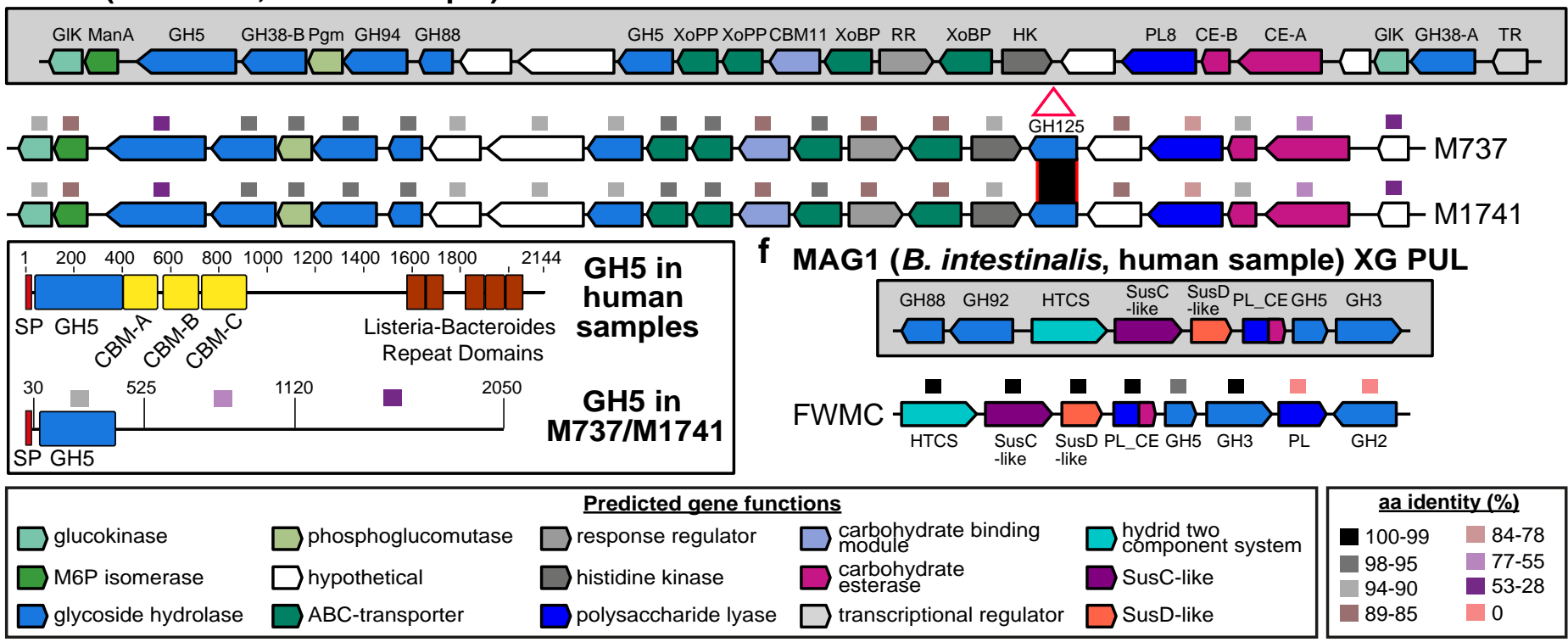

g

h

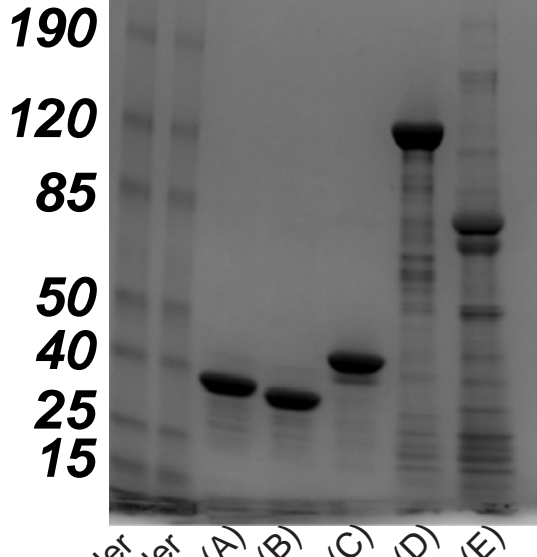

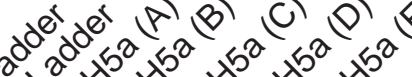

Bacteroidaceae

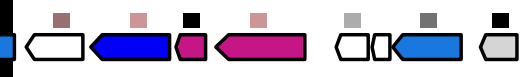

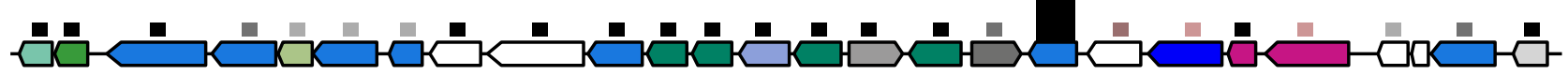

